\title{
Método Beam Search Aplicado a Problemas de Programação da Produção
}

\author{
José Eurípedes Ferreira de Jesus Filho
}

\author{
TESE APRESENTADA \\ $\mathrm{AO}$ \\ Instituto de Matemática e Estatística \\ DA \\ UNIVERSIDADE DE SÃo PAUlo \\ PARA \\ OBTENÇÃO DO TÍTULO \\ $\mathrm{DE}$ \\ DOUTOR EM CIÊNCIAS
}

\author{
Programa: Ciência da Computação \\ Orientador: Prof. Dr. Ernesto G. Birgin
}

Durante o desenvolvimento deste trabalho o autor recebeu auxílio financeiro da CAPES

São Paulo, agosto de 2018 


\title{
Método Beam Search Aplicado a Problemas de Programação da Produção
}

\author{
Esta versão da tese contém as correções e alterações sugeridas \\ pela Comissão Julgadora durante a defesa da versão original do trabalho, \\ realizada em 05/12/2018. Uma cópia da versão original está disponível no \\ Instituto de Matemática e Estatística da Universidade de São Paulo.
}

Comissão Julgadora:

- Prof. Dr. Ernesto G. Birgin (orientadora) - IME-USP

- Prof ${ }^{a}$. Dr ${ }^{a}$. Maristela Oliveira dos Santos - ICMC-USP

- Prof. Dr. Alexandre César Muniz de Oliveira - UFMA

- Prof. Dr. Ricardo Coelho Silva - UFC

- Prof. Dr. Cláudio Fabiano Motta Toledo - ICMC-USP 


\section{Agradecimentos}

Agradeço primeiramente a minha esposa Raissa que sempre esteve do meu lado em toda a minha jornada no doutorado, se sacrificando inúmeras vezes só para me ajudar a cumprir os meus prazos.

Agradeço ao meu filho Arthur por sempre querer o pai por perto, por fazer carinhas só para me convencer a brincar mais um pouco.

Agradeço a minha mãe Gilda pelas palavras de carinho e de ternura nos inúmeros acolhimentos aos meus problemas.

Agradeço ao meu pai José Eurípedes pela história de vida de luta que impactou diretamente na formação do meu caráter. Agradeço pelos inúmeros conselhos e pelo inestimável suporte.

Agradeço a minha irmã Juliana por carregar todo o fardo nas costas, mesmo sendo inúmeras vezes injustiçada, só para tornar minha formação acadêmica possível.

Agradeço aos pais da minha esposa, Moacir e Regina, por todo acolhimento, amizade e suporte oferecido. Estes dois foram em inúmeras vezes meus pais em São Paulo.

Agradeço também ao Professor Ernesto e a Professora Débora, os quais sem a orientação, paciência e devoção com certeza este trabalho não teria sido possível.

O presente trabalho foi realizado com apoio da Coordenação de Aperfeiçoamento de Pessoal de Nível Superior - Brasil (CAPES) - Código de Financiamento 001. 


\section{Resumo}

\section{FERREIRA, J. E. Método Beam Search Aplicado a Problemas de Programação da Pro-}

dução. 2018. Tese - Instituto de Matemática e Estatística, Universidade de São Paulo, São Paulo, 2018.

Nesta tese, dois diferentes problemas de programação da produção são abordados, o Flexible Job Shop Scheduling Problem com flexibilidade de sequenciamento e o Flowshop Scheduling Problem com tempos de espera e permutação de sequência. Para ambos, inicialmente um algoritmo list scheduling (LS) que explora características do problema é desenvolvido e então estendido para um método do tipo Beam Search (BS) que utiliza o LS em seus principais elementos: (1) expansão dos níveis, (2) avaliação local dos candidatos e (3) avaliação global dos candidatos. Todos os métodos propostos são determinísticos e seus pseudocódigos são cuidadosamente descritos para garantir a replicabilidade dos resultados reportados. O desempenho dos métodos propostos são avaliados utilizando instâncias e outros métodos heurísticos da literatura. Os resultados computacionais obtidos mostram a eficiência das heurísticas propostas que superaram os métodos da literatura utilizando pouco tempo computacional.

Palavras-chave: beam search, list scheduling, jobshop, jobshop flexível, flowshop. 


\section{Abstract}

FERREIRA, J. E. Método Beam Search Aplicado a Problemas de Programação da Produção. 2018. Tese - Instituto de Matemática e Estatística, Universidade de São Paulo, São Paulo, 2018.

In this thesis two diferent scheduling problems were addressed, the Flexible Job Shop Scheduling Problem with sequence flexibility and the Flowshop Scheduling Problem with waiting times and sequence permutation. For both problems, firstly, a list scheduling (LS) algorithm which exploit features of the problem was developed and then it was extedend to a Beam Search (BS) method which use the LS in his main features: (1) level expansion, (2) local evaluation and (3) global evaluation. All the proposed methods are deterministics and their pseudocodes are carefully described to ensure the replicability of the reported results. The performance of the proposed methods was evaluated using instances and other heuristic methods found in literature. The computational results show the efficiency of the proposed heuristics, which outperformed the literature methods while using low computational time.

Keywords: beam search, list scheduling, jobshop, flexible jobshop, flowshop. 


\section{Sumário}

$\begin{array}{ll}\text { Lista de Figuras } & \text { ix }\end{array}$

Lista de Tabelas $\quad$ xi

1 Introdução $\quad 1$

2 Flexible Job Shop Scheduling Problem com Flexibilidade de Sequenciamento 5

2.1 Modelagem matemática . . . . . . . . . . . . . . . 8

2.2 Algoritmo List Scheduling . . . . . . . . . . . . . . . . . . . . 9

2.2 .1 Características gerais . . . . . . . . . . . . . . . 10

2.2 .2 Soluções parciais . . . . . . . . . . . . . . . . . . . 10

2.2 .3 Regras de seleção . . . . . . . . . . . . . . . . . . . . . . 11

2.2 .4 Pseudo-código . . . . . . . . . . . . . . . . . . . . . . 13



2.3 Método Beam Search . . . . . . . . . . . . . . . . . . 16

2.3 .1 Estratégias de avaliação local e global . . . . . . . . . . . . . . . 17



2.4 Experimentos Numéricos . . . . . . . . . . . . . . . . . . . . . . 23





3 Flowshop Scheduling Problem com Tempos de Espera 35

3.1 Modelagem matemática . . . . . . . . . . . . . . . . . . 37

3.2 Algoritmo List Scheduling . . . . . . . . . . . . . . . . . . . . . . . . . . . . . . . . . . . . . . . . . . . . . . . . . . . . . .

3.3 Método Beam Search . . . . . . . . . . . . . . . . . . . . 45

3.4 Experimentos Numéricos . . . . . . . . . . . . . . . . 53



4 Conclusões $\quad 61$

4.1 Trabalhos Futuros . . . . . . . . . . . . . . . . . . . 61

$\begin{array}{ll}\text { Referências Bibliográficas } & 63\end{array}$ 
viii SUMÁRIO 


\section{Lista de Figuras}

2.1 Exemplo das restrições de precedências dadas por um DAG de uma possível tarefa do FJSP-SF (retirado de Birgin et al. (2015)) . . . . . . . . . . . . . . . . . . 6

2.2 Esquema ilustrativo de operações sem precedências entre si (as diferentes signatures e a capa) para a produção de um livro na indústria de impressão (retirado de Birgin et al. (2015)). . . . . . . . . . . . . . . . . . . . . . . . 6

2.3 DAG de precedências e tabela de tempos de processamento de um cenário do FJSPSF com $o=8$ operações e $m=2$ máquinas. . . . . . . . . . . . . . . . . . . . . 15

2.4 Pares operação/máquinas possíveis de serem programados considerando o cenário inicial da Figura 2.3. . . . . . . . . . . . . . . . . . . . . . . . . . 15

2.5 Conjunto de pares operação/máquina após a Regra 1 ser aplicada ao cenário descrito pela Figura 2.3. . . . . . . . . . . . . . . . . . . . . . . . . . . 15

2.6 Conjunto de pares operação/máquina após a Regra 1 e a Regra 2 serem aplicadas ao cenário descrito pela Figura 2.3. . . . . . . . . . . . . . . . . . . . . . 16

2.7 Gráfico de Gant da programação da operação $o_{1}$ na máquina $m_{1}$ considerando o cenário descrito pela Figura 2.3. . . . . . . . . . . . . . . . . . . . . . . 16

2.8 Árvore de busca após as Regras 1-3 serem aplicadas no cenário descrito pela Figura 2.3 e a programação do par $\left(o_{1}, m_{1}\right)$. . . . . . . . . . . . . . . . . 16

2.9 Representação gráfica das precedências entre as operações da instância YFJS02. . . 23

2.10 Representação gráfica das precedências entre as operações da instância DAFJS28. . . 26

2.11 Representação gráfica do desempenho do método Beam Search, com diferentes escolhas de parâmetros $\alpha, \beta$ e $\xi$, quando comparados aos resultados obtidos pelo solver CPLEX executado com limite de tempo de 1 hora. (a) Instâncias YFJS01-YFJS20.

(b) Instâncias DAFJS01-DAFJS30 . . . . . . . . . . . . . . . . . . . . . . 33

2.12 Representação gráfica do tempo de CPU em segundos requerido pelo método Beam Search, com diferentes escolhas de parâmetros $\alpha, \beta$ e $\xi$. (a) Instâncias YFJS01-YFJS20.

(b) Instâncias DAFJS01-DAFJS30. . . . . . . . . . . . . . . . . . . . . . . . 34

3.1 Programação da tarefa $J_{1}$ nas máquinas $M_{1}$ e $M_{2}$ de acordo com o problema descrito na Tabela 3.3. A parte hachurada representa a janela provocada pela programação. $\quad 39$

3.2 Programação das tarefas $J_{1}$ e $J_{2}$ nas máquinas $M_{1}$ e $M_{2}$ de acordo com o problema descrito na Tabela 3.3. A parte hachurada representa o restante da janela de $J_{1}$. . . 40

3.3 Programação das tarefas $J_{1}$ e $J_{3}$ nas máquinas $M_{1}$ e $M_{2}$ de acordo com o problema descrito na Tabela 3.3. A parte hachurada representa o restante da janela de $J_{1}$. . . 40 
3.4 Programação das tarefas $J_{1}$ e $J_{3}$ nas máquinas $M_{1}$ e $M_{2}$ de acordo com o problema descrito na Tabela 3.3. A parte hachurada representa o restante da janela de $J_{1} \ldots$. . 40

3.5 Programação das tarefas $J_{2}$ e $J_{3}$ nas máquinas $M_{1}$ e $M_{2}$ de acordo com o problema descrito na Tabela 3.3. A parte hachurada representa a janela de $J_{2}$. . . . . . . . . 41

3.6 Duas programações possíveis considerando o cenário descrito na Tabela 3.3 e data de entrega comum $d=10$. A programação de baixo é o resultado de empurrar as tarefas $J_{2}$ e $J_{3}$ dois instantes de tempo na programação de cima. Este processo mantêm as somas dos adiantamentos e dos atrasos mas diminui a soma dos tempos de espera. . 41

3.7 Três programações possíveis para a sequência de tarefas $J_{1}, J_{2}, J_{3}$ considerando o cenário descrito na Tabela 3.4 e data de entrega comum $d=7$. A programação (a) é o resultado da chamada do Algoritmo 7 sem o processo de empurrar. A programação (b) é o resultado da chamada com o processo de empurrar. A programação (c) é a programação ótima para o FSP-WT. . . . . . . . . . . . . . . . . . . 45

3.8 Três programações ótimas para a soma dos adiantamentos e atrasos considerando o cenário descrito na Tabela 3.5 e data de entrega comum $d=7$ mas com diferentes soma dos tempos de espera. A programação (a) é o resultado da programação da sequência $J_{1}, J_{3}, J_{2}$ utilizando o Algoritmo 7 sem o processo de empurrar. A programação (b) é o resultado da programação da sequência $J_{1}, J_{3}, J_{2}$ utilizando o Algoritmo 7 com o processo de empurrar. A programação (c) é o resultado da programação da sequência $J_{1}, J_{2}, J_{3}$ utilizando o Algoritmo $7 \mathrm{com}$ o processo de empurrar. (c) é ótimo para o FSP-WT. . . . . . . . . . . . . . . . . . . . . 46

3.9 Esquema da expansão do nível 1 para o nível 2 na árvore de busca do BS. Cada nó pai possui três filhos candidatos para a expansão. . . . . . . . . . . . . . . . . 47

3.10 Aplicação da busca local com $\alpha=2$ no esquema ilustrado pela Figura 3.9. Cada nó pai possui três filhos candidatos para a expansão. Os filhos candidatos são avaliados pela busca local e somente os dois melhores (nós hachurados) são escolhidos para serem avaliados pela busca global. . . . . . . . . . . . . . . . . . . 47

3.11 Aplicação da busca global com $\beta=2$ no esquema ilustrado pela Figura 3.10. Cada nó pai possui três filhos candidatos para a expansão. Os filhos candidatos escolhidos pela busca local são avaliados pela busca global e somente os dois melhores (nós coloridos) são mantidos na árvore para a próxima iteração do método. . . . . . . . . 48

3.12 Tempo computacional médio em segundos gasto pelo BS com diferentes configurações de $\alpha$ e $\beta$ para solucionar uma instância. . . . . . . . . . . . . . . 57 


\section{Lista de Tabelas}

2.1 Descrição das instâncias com $Y$-jobs. . . . . . . . . . . . . . . . 24

2.2 Descrição das instâncias nas quais as relações de precedências entre as operações são dadas por um DAG qualquer. . . . . . . . . . . . . . . . . . 25

2.3 Resultados numéricos da aplicação do CPLEX ao modelo (2.1-2.8) com as instâncias YFJS01-YFJS20. Como em Birgin et al. (2014), uma solução viável inicial é computada com a heurística EST (Birgin et al., 2014) e passada ao CPLEX. . . . . . 27

2.4 Resultados numéricos da aplicação do CPLEX ao modelo (2.1-2.8) com as instâncias DAFJS01-DAFJS30. Como em Birgin et al. (2014), uma solução factível inicial é computada com a heurística EST Birgin et al. (2014) e passada ao CPLEX. . . . . . 28

2.5 Resultados do algoritmo list scheduling aplicado ao conjunto de instâncias YFJS01-

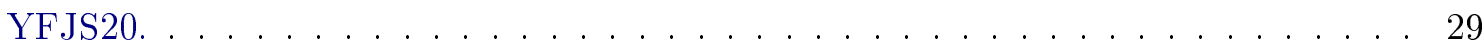

2.6 Resultados do algoritmo list scheduling aplicado ao conjunto de instâncias DAFJS01-



2.7 Médias dos desvios relativos obtidos na comparação dos resultados do método Beam Search, com diferentes escolhas de parâmetros para $\alpha, \beta$, e $\xi$, em relação ao CPLEX solver com dois limites de tempo de CPU (1 hora e 10 horas), quando aplicados às instâncias YFJS01-YFJS20. . . . . . . . . . . . . . . . . . . . 31

2.8 Médias dos desvios relativos obtidos na comparação dos resultados do método Beam Search, com diferentes escolhas de parâmetros para $\alpha, \beta$, e $\xi$, em relação ao CPLEX solver com dois limites de tempo de CPU (1 hora e 10 horas), quando aplicados às instâncias DAFJS01-DAFJS30． . . . . . . . . . . . . . . . 32

2.9 Erros relativos obtidos pelo método Beam Search aplicado à vários conjuntos clássicos de instâncias do FJSP. . . . . . . . . . . . . . . . . . . . . . 32

2.10 Melhores erros relativos obtidos por algoritmos genéticos clássicos executados cinco vezes em cada conjunto de instância do FJSP. . . . . . . . . . . . . . . . . . . . . 32

3.1 Variáveis do modelo matemático do FSP-WT. . . . . . . . . . . . . . . . . 37

3.2 Parâmetros do modelo matemático do FSP-WT. . . . . . . . . . . . . . . . 37

3.3 Tempos de processamento para um problema com $n=3$ tarefas e $m=2$ máquinas. . 39

3.4 Tempos de processamento para um problema com $n=3$ tarefas e $m=3$ máquinas. . 44

3.5 Tempos de processamento para um problema com $n=3$ tarefas e $m=3$ máquinas. . 44 
3.6 Resultados computacionais para o LS proposto neste trabalho considerando a data de entrega $d^{\prime}$. Na tabela, cada entrada de $E / T$ é a média dos $I D R$ da soma dos adiantamentos e dos atrasos para 50 instâncias com $n$ tarefas e $m$ máquinas. \# Empates é a quantidade de vezes que dois ou mais métodos encontraram o melhor valor de $E / T$. $W$ é o $I D R$ da soma dos tempos de espera nos casos em que houveram

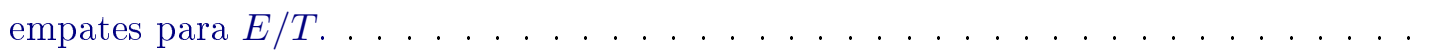

3.7 Resultados computacionais para o LS proposto neste trabalho considerando a data de entrega $d^{\prime \prime}$. Na tabela, cada entrada de $E / T$ é a média dos $I D R$ da soma dos adiantamentos e dos atrasos para 50 instâncias com $n$ tarefas e $m$ máquinas. \# Empates é a quantidade de vezes que dois ou mais métodos encontraram o melhor valor de $E / T$. $W$ é o $I D R$ da soma dos tempos de espera nos casos em que houveram

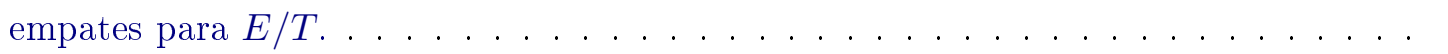

3.8 Resultados computacionais preliminares para o BS com diferentes valores para os parâmetros $\alpha$ e $\beta$ utilizando um conjunto reduzido de instâncias e data de entrega $d^{\prime}$. Na tabela, $E / T$ é a média dos $I D R$ da soma dos adiantamentos e dos atrasos encontrados pela BS em relação às demais configurações do método. $W$ é o $I D R$ da soma dos tempos de espera nos casos em que uma ou mais configurações encontraram o mesmo valor de $E / T \ldots \ldots \ldots \ldots \ldots \ldots \ldots$

3.9 Resultados computacionais preliminares para o BS com diferentes valores para os parâmetros $\alpha$ e $\beta$ utilizando um conjunto reduzido de instâncias e data de entrega $d^{\prime \prime}$. Na tabela, $E / T$ é a média dos $I D R$ da soma dos adiantamentos e dos atrasos encontrados pela BS em relação às demais configurações do método. $W$ é o $I D R$ da soma dos tempos de espera nos casos em que uma ou mais configurações encontraram o mesmo valor de $E / T \ldots \ldots \ldots \ldots \ldots \ldots \ldots$

3.10 Resultados computacionais dos métodos LS e BS propostos neste capítulo considerando as 1200 instâncias e a data de entrega $d^{\prime}$. Na tabela, cada entrada de $\overline{g a p}$ é a média dos gap relativos da soma dos adiantamentos e dos atrasos encontrados pelos métodos em relação aos resultados reportados por Chandra et al. (2009) em 50 instâncias com $n$ tarefas e $m$ máquinas. \# Empates é a quantidade de vezes que os dois métodos encontraram o mesmo valor de gap. $W$ é o $I D R$ da soma dos tempos de espera nesses casos. \# Vitórias é a quantidade de vezes em que o método conseguiu atingir ou superar os resultados reportados por Chandra et al. (2009). . . . . . . . . .

3.11 Resultados computacionais dos métodos LS e BS propostos neste capítulo considerando as 1200 instâncias e a data de entrega $d^{\prime \prime}$. Na tabela, cada entrada de $\overline{g a p}$ é a média dos gap relativos da soma dos adiantamentos e dos atrasos encontrados pelos métodos em relação aos resultados reportados por Chandra et al. (2009) em 50 instâncias com $n$ tarefas e $m$ máquinas. \# Empates é a quantidade de vezes que os dois métodos encontraram o mesmo valor de gap. $W$ é o $I D R$ da soma dos tempos de espera nesses casos. \# Vitórias é a quantidade de vezes em que o método conseguiu atingir ou superar os resultados reportados por Chandra et al. (2009). . . . . . . . . 
3.12 Resultados computacionais dos métodos LS e BS propostos neste capítulo comparados com um solver ótimo executado sobre (3.1)-(3.10) considerando as instâncias de pequeno porte e a data de entrega $d^{\prime}$. Na tabela, cada entrada de $\overline{g a p}$ é a média dos gap relativos da soma dos adiantamentos e dos atrasos encontrados pelos métodos em relação aos resultados encontrados pelo solver ótimo em 50 instâncias com $n$ tarefas e $m$ máquinas. \# Empates é a quantidade de vezes que os dois métodos encontraram o mesmo valor de gap. $W$ é o $I D R$ da soma dos tempos de espera nesses casos. \# Vitórias é a quantidade de vezes em que o método conseguiu gap igual a zero. . . . . 60

3.13 Resultados computacionais dos métodos LS e BS propostos neste capítulo comparados com um solver ótimo executado sobre (3.1)-(3.10) considerando as instâncias de pequeno porte e a data de entrega $d^{\prime \prime}$. Na tabela, cada entrada de $\overline{g a p}$ é a média dos gap relativos da soma dos adiantamentos e dos atrasos encontrados pelos métodos em relação aos resultados encontrados pelo solver ótimo em 50 instâncias com $n$ tarefas e $m$ máquinas. \# Empates é a quantidade de vezes que os dois métodos encontraram o mesmo valor de gap. W é o IDR da soma dos tempos de espera nesses casos. \# Vitórias é a quantidade de vezes em que o método conseguiu gap igual a zero. . . . . 60 


\section{Capítulo 1}

\section{Introdução}

No início do século XX, Frederick Winslow Taylor já observava que a ineficiência e a ociosidade do uso do tempo produtivo no nível operacional da indústria onde trabalhava reduziam a produtividade total para cerca de um terço do que deveria ser. Essas minuciosas observações de Taylor, mais tarde, foram as bases para a criação da chamada Administração Científica que tinha como principal objetivo eliminar os desperdícios e consequentemente aumentar a produtividade das empresas por meio da aplicação de métodos e técnicas da engenharia industrial. Desde então, é evidente a preocupação com a eficiência do nível operacional das indústrias, visto que o mercado se torna cada vez mais competitivo, exigindo constante aumento na produtividade, redução de custos e encurtamento de prazos de entrega (Chiavenato, 2004). Neste contexto, o planejamento das tarefas de uma empresa deve ser feito de forma a aproveitar os recursos disponíveis da melhor maneira possível. A este planejamento, dá-se o nome de Planejamento e Controle da Produção (PCP). Surgiram portanto, várias técnicas para as tomadas de decisões do PCP. Estas técnicas podem ser classificadas em dois grupos que usam diferentes conceitos de administração da produção: Just In Case (JIC) e Just In Time (JIT) (Antunes Júnior et al., 1989).

Na JIC, o planejamento e a organização das tarefas visam minimizar o tempo ocioso dos meios de produção envolvidos através de uma previsão de mercado. Assim, é comum gerar estoques para atendimento de demandas futuras. Na JIT, o principal objetivo é minimizar todos os custos envolvidos que não agregam valor ao produto como o de estocagem, tempo/custo de troca nas máquinas de produção (setup), tempo/custo na movimentação de matérias primas e intermediárias, etc. Contudo, tanto na JIC quanto na JIT, as decisões de o quê, quando, onde, como, com o quê e o quanto produzir devem ser tomadas e o PCP deve atender as demandas no prazo e respeitar as restrições de capacidade e os recursos disponíveis no meio produtivo além de, geralmente, minimizar os custos envolvidos.

O presente trabalho desenvolve métodos heurísticos para solucionar dois diferentes cenários de programação da produção: o Flexible Job Shop Scheduling Problem com flexibilidade de sequenciamento (FJSP-SF) e o Flowshop Scheduling Problem com tempos de espera (FSP-WT).

O Job Shop Scheduling Problem (JSP) clássico consiste no processamento de $n$ tarefas em um ambiente com $m$ máquinas. Cada tarefa é composta por operações (cada uma das quais deve ser processada numa máquina predefinida) que possuem uma estrutura linear de precedência de processamento entre si. Portanto, cada tarefa possui uma única rota de processamento possível através das $m$ máquinas e a rota de uma dada tarefa pode ser diferente da rota de outra tarefa. O Flexible Job Shop Scheduling Problem (FJSP) é uma generalização do JSP na qual uma operação pode ser processada por várias máquinas não necessariamente idênticas. Portanto, cada tarefa possui várias possibilidades de rotas de processamento através das $m$ máquinas. O objetivo é determinar em qual máquina cada operação será processada e a ordem de processamento das operações nas máquinas levando em consideração a otimização de algum critério. O Flexible Job Shop Scheduling Problem com flexibilidade de sequenciamento (FJSP-SF) é uma extensão do FJSP que permite que a estrutura de precedências entre as operações de uma mesma tarefa seja estabelecida por um grafo direcionado acíclico (DAG) qualquer ao invés de uma estrutura linear. Assim, o problema consiste em 
alocar as operações a pelo menos uma dentre as possíveis máquinas de processamento e determinar o tempo de início de processamento destas operações nas máquinas, respeitando as precedências de acordo com o DAG, de forma que o processamento das operações não se sobreponham.

O Flowshop Scheduling Problem (FSP) clássico também consiste no processamento de $n$ tarefas em um ambiente de $m$ máquinas. Contudo, diferentemente do JSP, todas as tarefas devem ser processadas obedecendo uma mesma rota nas máquinas de processamento, quer dizer, a $k$-ésima operação de cada tarefa deve ser processada pela $k$-ésima máquina do sistema, com $k=1, \ldots, m$. Pinedo (2008) menciona que nestes tipos de problemas, assume-se que as máquinas de processamento estão posicionadas em série e isto caracteriza o ambiente como flow shop. Neste problema, a sequência do processamento das $n$ tarefas pode mudar de uma máquina para outra. Pinedo (2008) chama de permutation flowshops os cenários em que a sequência de processamento das tarefas nas máquinas é obrigatoriamente a mesma. O Flowshop Scheduling Problem com tempos de espera (FSP-WT) é uma variante do FSP em que os tempos entre o término do processamento de uma tarefa em uma máquina até o início de processamento na próxima máquina são considerados. Dessa forma, o problema consiste em determinar uma única sequência de processamento das tarefas em todas as máquinas, atribuindo instantes de início de processamento para cada uma das operações do sistema sem sobreposição, levando em conta os tempos de espera das tarefas nas máquinas e considerando a otimização de algum critério.

Este trabalho propõe métodos heurísticos do tipo list scheduling (LS) e Beam Search (BS) desenvolvidos para a minimização do makespan no FJSP-SF (Birgin et al., 2015) e para a minimização da somatória do earliness e do tardiness no FSP-WT com permutação de sequências e data de entrega única. Em ambos os cenários, inicialmente o LS é cuidadosamente desenvolvido e então é usado como base na construção do BS. Para o FJSP-SF, o presente trabalho apresenta ainda uma melhoria que aleatoriza a construção de soluções parciais nos arcabouços do método Beam Search.

Métodos heurísticos ganharam muito destaque desde o desenvolvimento da teoria da complexidade, na década de 70 , e da prova de que a maioria dos problemas combinatoriais são NPDifícil (Gendreau, 2003). Nesta linha, o uso de heurísticas como abordagem para o FJSP-SF e para o FSP-WT é encorajado devido à complexidade de respectivos problemas. Garey et al. (1976); Lenstra e Kan (1979) mostram que o JSP é NP-Difícil e uma vez que o JSP é um caso particular do FJSP-SF, tem-se que o FJSP-SF também é NP-Difícil. Similarmente, Hall e Posner (1991) mostram que o FSP com uma única máquina é NP-Difícil e portanto o FSP-WT também é NP-Difícil.

De acordo com Rakrouki et al. (2012), o método Beam Search consiste de uma heurística que mantêm a estrutura da árvore de busca do Branch and Bound e realiza podas baseadas em alguma regra heurística, geralmente gulosa. Através dessas podas, muitas soluções são descartadas e a complexidade do algoritmo diminui consideravelmente. Lowerre (1976) foi o primeiro a utilizar o método Beam Search em um trabalho de reconhecimento de linguagens. Desde então, o método Beam Search foi utilizado em vários trabalhos. Nesta linha, podem ser citados os trabalhos de Wang e Lim (2007), Valente e Alves (2004), Akeb et al. (2009), Blum et al. (2009) e Akeb et al. (2011), Fernandez-Viagas e Framinan (2017), Mejía e Niño (2017), Vélez-Gallego et al. (2016), entre outros.

No método Beam Search clássico somente alguns nós da árvore de busca, ditos mais promissores, são mantidos para o passo de ramificação. A quantidade de nós mantidos é chamada de largura do beam $\beta$. A avaliação dos nós a serem selecionados pode ser feita de diversas maneiras, desde regras heurísticas até funções de avaliação parcial ou completa. Com o decorrer dos anos, novas técnicas de avaliação deram origem a outros tipos de Beam Search, como o Filtered Beam Search. Este último caracteriza-se por fazer a avaliação dos nós em dois estágios. No primeiro estágio, o conjunto de nós é filtrado e reduzido a partir de uma regra mais simples e no segundo estágio uma avaliação mais complexa e abrangente é aplicada somente neste conjunto reduzido (Valente e Alves, 2004).

Algoritmos list scheduling são algoritmos simples de escalonamento que a cada iteração selecionam uma única operação para ser programada baseados em algum critério local. Inúmeros trabalhos em diversas áreas utilizam algoritmos LS como abordagem. Como exemplo, em trabalhos recentes, podem ser citados os trabalhos de Agnetis et al. (2014), Tang et al. (2010), Fard et al. 
(2014). Agnetis et al. (2014) apresenta um método para um problema de programação de tarefas onde cada tarefa possui uma chance de sucesso e uma recompensa caso seja completada. Tang et al. (2010) e Fard et al. (2014) abordam problemas de distribuição de cargas em sistemas distribuídos. Além destes trabalhos, Sabuncuoglu e Bayiz (1999) utilizaram diferentes LS da literatura para estimar a qualidade de soluções parciais obtidas por um algoritmo BS para o JSP. Neste trabalho, os melhores candidatos eram escolhidos para a fase de expansão de um BS e a expansão do nível era feita utilizando dois outros métodos chamados active e nondelay sobre os candidatos mais promissores. O BS da presente tese utiliza um LS que explora características específicas do FJSP-SF e do FSP-WT não somente para avaliar as soluções parciais, mas também para expandir os níveis e filtrar os nós candidatos.

Este trabalho está organizado como segue. O Capítulo 2, descreve em detalhes o FJSP-SF e define os métodos list scheduling e Beam Search para o problema. Os resultados computacionais obtidos pelos dois métodos utilizando instâncias do FJSP-SF da literatura também são apresentados em adição com alguns experimentos utilizando instâncias clássicas do FJSP. O Capítulo 3 descreve em detalhes o FSP-WT e então desenvolve os métodos list scheduling e Beam Search utilizando a mesma metodologia empregada no Capítulo 2. Por fim, os resultados computacionais para ambos os métodos são reportados e comparados com outros métodos heurísticos da literatura. As conclusões gerais e as propostas para trabalhos futuros desta tese são então apresentadas no Capítulo 4. 


\section{Capítulo 2}

\section{Flexible Job Shop Scheduling Problem com Flexibilidade de Sequenciamento}

O Job Shop Scheduling Problem (JSP) clássico é um problema onde deve-se escalonar o processamento de $n$ tarefas em um ambiente com $m$ máquinas. Cada tarefa é composta por várias operações (geralmente $m$ operações) organizadas em uma estrutura linear de precedência entre si o que significa que uma operação de uma tarefa não pode iniciar processamento antes que a operação que a antecede termine de ser processada. Portanto, cada tarefa possui uma única rota de processamento possível através das $m$ máquinas. O Flexible Job Shop Scheduling Problem (FJSP) consiste em uma extensão do JSP clássico onde as operações possuem várias máquinas nas quais podem ser processadas. As máquinas de processamento não são necessariamente idênticas e isso implica em diferentes tempos de processamento mesmo considerando uma mesma operação. Dessa forma, diferentemente do JSP clássico, existem várias possibilidades de rotas de processamento para cada tarefa através das $m$ máquinas do ambiente. O objetivo do problema é determinar em qual máquina cada operação será processada e a ordem do processamento das operações associadas à cada uma das máquinas considerando a otimização de algum critério. O Flexible Job Shop Scheduling Problem com flexibilidade de sequenciamento (FJSP-SF), por sua vez, é uma variante do FJSP que permite que a estrutura de precedências entre as operações de uma mesma tarefa seja estabelecida por um grafo direcionado acíclico (DAG) qualquer ao invés de uma estrutura linear. Quer dizer, uma operação pode tanto suceder quanto preceder mais de uma operação. Assim, o problema consiste em alocar as operações às possíveis máquinas de processamento e em sequenciar o processamento destas operações nas máquinas respeitando as precedências de acordo com o DAG. O critério adotado nos métodos apresentados neste capítulo foi a minimização do instante de término do processamento da última operação (makespan). Foi considerado ainda que o tempo de processamento de cada operação em uma máquina é um dado conhecido a priori e que o processamento de uma operação não pode ser interrompido até que seja finalizado.

Um exemplo de tarefa com este tipo de estrutura de precedência é mostrado na Figura 2.1. Este problema pode ser encontrado na prática em ambientes industriais, como nas indústrias de impressão (Zeng et al., 2010), onde operações de montagem e desmontagem fazem parte do processo produtivo. A impressão de um livro, por exemplo, pode ser dividida em três tarefas principais: pré-impressão, impressão e pós-impressão (PNEAC, 2015). A pré-impressão é formada por cinco etapas distintas. A impressão geralmente é realizada através de seis processos separados. A pósimpressão consiste em quatro processos. Além disso, geralmente, existem algumas pequenas tarefas de finalização de pós-impressão. Estas três tarefas principais do processo de impressão como um todo possuem restrições de precedências. Contudo, dentro destas três tarefas existem operações que não possuem precedências entre si. As páginas de um livro, por exemplo, são divididas em signatures de 8,16 ou 32 páginas individuais que podem ser impressas, cortadas e dobradas separadamente. A capa do livro também é um elemento que pode ser preparado separadamente. Finalmente, todos os elementos devem ser compostos para formar o livro. A Figura 2.2 ilustra o processo de impressão e composição de um livro. É fácil ver que a flexibilidade nas precedências das operações do processo de 
impressão pode ser encontrado em várias aplicações industriais, tornando o FJSP-SF um problema com uma ampla variedade de aplicações.

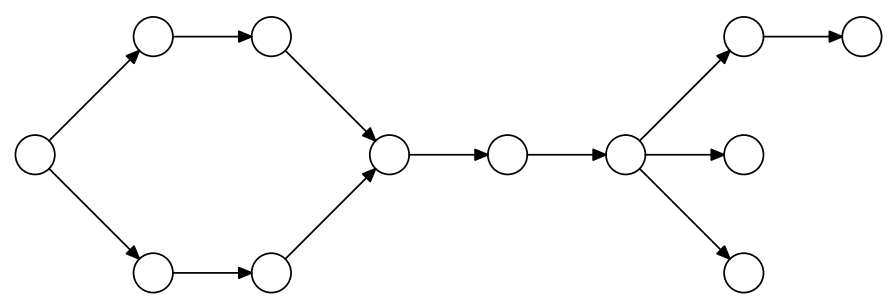

Figura 2.1: Exemplo das restrições de precedências dadas por um DAG de uma possível tarefa do FJSP-SF (retirado de Birgin et al. (2015)).



A book, as many other products of the printing industry, is composed by several parts that can be processed independently



Figura 2.2: Esquema ilustrativo de operações sem precedências entre si (as diferentes signatures e a capa) para a produção de um livro na indústria de impressão (retirado de Birgin et al. (2015)).

Considerando as variações do JSP clássico, que muitas vezes surgem a partir da adaptação do JSP para contextos mais próximos da realidade industrial, a flexibilização de uma operação poder ser processada por um subconjunto das máquinas disponíveis é dita como flexibilidade de roteamento. Esta flexibilização transforma o JSP clássico em FJSP. A flexibilidade de sequenciamento consiste na possibilidade de representar as precedências entre as operações de uma mesma tarefa através de um DAG qualquer. Este trabalho aborda o FJSP com flexibilidade de sequenciamento (FJSP-SF). Poucos trabalhos da literatura lidam com a variação do FJSP com flexibilidade de sequenciamento. No trabalho de Kim et al. (2003) foi proposto um algoritmo evolucionário simbiótico para um JSP com flexibilidade de roteamento, sequenciamento e processamento. Flexibilidade de processamento é a possibilidade de completar uma tarefa, ou produzir um produto, a partir de operações ou sequência de operações diferentes. O trabalho de Lee et al. (2012) lida com um FJSP com flexibilidade de sequenciamento na qual as precedências são do tipo AND/OR. Um problema de sequenciamento de tarefas de uma fábrica de moldes é descrito em Gan e Lee (2002). No problema, vários tipos de flexibilidades, incluindo flexibilidade de sequenciamento, são tratadas de maneira integrada pelo 
método proposto.

O FJSP-SF é tido como NP-Difícil uma vez que ele tem o JSP, que é conhecido como NPDifícil (Garey et al., 1976; Lenstra e Kan, 1979), como um caso particular. Um exemplo do desafio matemático provido pelo JSP clássico é o cenário contendo 10 máquinas e 10 tarefas proposto no trabalho de Fisher e Thompson (1963) que foi debatido e estudado por vários pesquisadores e sua solução só apareceu 20 anos mais tarde. Além disso, Nuijten e Aarts (1996) mostraram que o FJSP está entre os mais difíceis problemas combinatoriais conhecidos. Em contrapartida, as decisões da programação das operações nas máquinas dentro das indústrias geralmente devem ser tomadas em curtos espaços de tempo.

Devido à esta complexidade computacional, somente alguns trabalhos centrados em solucionar o FJSP de forma exata podem ser encontrados na literatura. Nesta linha, Fattahi et al. (2007) propuseram um modelo Linear de Programação Inteiro Misto (MIP) como abordagem para o FJSP e solucionaram instâncias de pequeno porte com um software comercial. Mais tarde, Özgüven et al. (2010) introduziram um MIP mais conciso para o FJSP. Birgin et al. (2014) propuseram um novo MIP que incorpora a flexibilidade de sequenciamento ao FJSP e avaliaram o seu desempenho utilizando instâncias baseadas em cenários da indústria de impressão e também utilizando instâncias para o FJSP e JSP da literatura. De acordo com os experimentos, o CPLEX com o novo MIP obteve resultados melhores quando comparados aos resultados obtidos em Özgüven et al. (2010).

$\mathrm{Na}$ literatura, é possível encontrar vários trabalhos que utilizam heurísticas para minimização do makespan no FJSP. Nesta linha, Brandimarte (1993) é um dos pioneiros e em seu trabalho utiliza uma abordagem de duas fases. Na primeira fase, uma estratégia heurística chamada regra de despacho atribui as operações às máquinas de processamento. Na segunda fase, a definição do sequenciamento das operações nas máquinas é feita utilizando busca tabu. Este tipo de abordagem, de duas fases, é denominado abordagem hierárquica.

Dauzère-Pérès e Paulli (1997) e Mastrolilli e Gambardella (2000) utilizaram busca tabu (TS) para solucionar o FJSP em uma única fase, quer dizer, o método define em quais máquinas as operações serão processadas e o sequenciamento das operações nas máquinas de forma integrada. O algoritmo genético (GA) proposto por Ho et al. (2007), denominado learnable genetic architecture (LEGA), também aborda o FJSP de maneira integrada utilizando um módulo de aprendizagem que influencia a diversidade e a qualidade da população combinado à abordagem evolutiva do GA. Pezzella et al. (2008) desenvolveram um GA tradicional com alguns componentes melhorados da literatura e com uma mutação de atribuição, denominada mutação inteligente. De acordo com os experimentos computacionais, o GA superou os resultados de outros GAs da literatura e obteve soluções competitivas quando comparadas com as soluções da TS de Mastrolilli e Gambardella (2000). Gutiérrez e García-Magariño (2011) propuseram um GA híbrido que soluciona o FJSP de forma hierárquica. Inicialmente, o GA faz atribuições das operações nas máquinas de processamento sem a garantia de viabilidade. Posteriormente, uma heurística de reparo é aplicada às soluções para minimizar as violações. Além disso, a heurística também calcula a qualidade da solução, funcionando assim, no contexto dos GAs, como função de reparo e de fitness. Uma grande quantidade de comparações com trabalhos recentes da literatura, incluindo os resultados de Mastrolilli e Gambardella (2000) e de Brandimarte (1993), foram feitas para mostrar o desempenho do GA. Yuan e Xu (2012) desenvolveram um algoritmo de dois estágios para solucionar o FJSP. No primeiro estágio, um algoritmo evolucionário denominado hybrid harmony search (HHS) é executado até que uma solução que não se é possível realizar melhorias seja encontrada. No segundo estágio, um algoritmo baseado em programação com restrições denominado large neighborhood search (LNS) é utilizado para refinar a solução obtida pelo HHS no primeiro estágio. Utilizando quatro conjuntos de instâncias da literatura os autores concluíram que o HHS/LNS tem um desempenho bastante competitivo quando comparado com outros métodos da literatura. Wang et al. (2012) apresentaram um algoritmo de Colônia Artificial de Abelhas como abordagem para o FJSP utilizando algumas estratégias para balancear a diversificação e a intensificação no processo de busca. Nesse trabalho, para evitar a convergência prematura do método, abelhas sentinelas que controlam o comportamento da busca são adicionadas no processo de atualização da população de abelhas. O desempenho do método foi 
avaliado utilizando instâncias da literatura e se mostrou efetivo quando comparado a outros métodos. Bouazza et al. (2017) utilizam algumas técnicas avançadas de Q-Learning como abordagem para o FJSP parcial. Nesse trabalho, os autores usam alguns "produtos inteligentes" que coletam informações e aprendem a decidir quais as melhores regras de seleção de operação e regras de despacho para realizar a programação de uma dada operação. Bozejko et al. (2012) desenvolveram uma busca tabu utilizando paralelismo em GPU que soluciona o FJSP através da exploração paralela de diversas vizinhanças. No trabalho, inicialmente várias vizinhanças são geradas através de um processo mestre controlado pela CPU e então atribuídas à várias GPUs onde serão exploradas seguindo as regras da busca tabu. Depois de algumas iterações, as soluções são coletadas pelo processo mestre e a melhor dentre elas é então escolhida para a geração das novas vizinhanças.

A presente tese, inicialmente, constrói um método heurístico do tipo list scheduling (LS) que soluciona o FJSP-SF e a partir disso, desenvolve um método Beam Search (BS) baseado nas regras utilizadas pelo LS.

O método Beam Search é uma heurística que realiza enumeração em árvore, semelhante ao método Branch and Bound, que utiliza critérios heurísticos para realizar podas (Rakrouki et al., 2012). A primeira utilização do método BS foi em um trabalho de reconhecimento de linguagens, feito por Lowerre (1976). Mais tarde, Rubins (1978) modificou a maneira conhecida de representação dos pixels para utilizar um BS como método para solucionar um problema de reconhecimento de imagens. Foi neste trabalho que o nome Beam Search se popularizou.

No trabalho de Sabuncuoglu e Bayiz (1999) um método BS foi usado como abordagem para o JSP clássico. Neste trabalho, dois métodos heurísticos, chamados active e nondelay, eram utilizados para a expansão dos níveis na árvore. A qualidade de cada nó era então estimada através de vários LSs da literatura e para cada nó pai, somente o nó filho mais promissor era então mantido.

Este capítulo aborda o FJSP-SF tratado nesta tese e está fortemente baseado em Birgin et al. (2015). Nesta linha, a Seção 2.1 apresenta em detalhes o modelo matemático para este problema. A Seção 2.2 descreve cuidadosamente o algoritmo LS proposto, definindo o conceito de soluções parciais e três regras de seleção de operações para serem programadas e por fim apresentando o pseudocódigo do algoritmo. A Seção 2.3 desenvolve o método BS para o FJSP-SF, descrevendo a origem do método a partir do algoritmo LS. Em seguida então, o pseudocódigo do método BS também é apresentado na Seção 2.3. As Seções 2.4 e 2.5 apresentam os experimentos numéricos realizados e discutem os resultados obtidos.

As principais vantagens dos métodos apresentados neste capítulo são: (a) tempo finito de término de processamento; (b) dependentes de um pequeno conjunto de parâmetros (o LS não possui nenhum parâmetro enquanto o Beam Search possui apenas três); (c) regras de desempates precisamente descritas; (d) resultados computacionais promissores.

\subsection{Modelagem matemática}

Seja $n$ o número de tarefas, o o número de operações e $m$ o número de máquinas. O número de tarefas não tem um papel explícito no modelo, mas é utilizado para entendimento. Para cada operação $i(i=1, \ldots, o)$, seja $F_{i} \subseteq\{1,2, \ldots, m\}\left(F_{i} \neq \emptyset\right)$ o subconjunto de máquinas que pode processar a operação $i$ com tempo de processamento $p_{i k}, k \in F_{i}$. Além disso, seja $A$ o conjunto de pares $(i, j)$ com $i, j \in\{1, \ldots, o\}$ tal que se $(i, j)$ pertence ao conjunto $A$, então a operação $i$ precede a operação $j$, quer dizer, a operação $j$ não pode iniciar processamento antes do término do processamento da operação $i$. O conjunto $A$ define implicitamente as tarefas do problema uma vez que existem precedências somente entre operações de uma mesma tarefa. $\mathrm{O}$ conjunto $A$ define ainda um grafo direcionado acíclico (DAG) onde os vértices estão nomeados de 1 até $o$ e as arestas são os pares $(i, j)$. Dizemos que o problema está bem definido se o DAG definido pelo conjunto $A$ não possui ciclos. O problema consiste em atribuir cada operação $i$ à uma máquina $k \in F_{i}$ e determinar o tempo de início de processamento $s_{i}$ que satisfaz as precedências dadas pelo conjunto $A$. Uma máquina só pode processar uma operação por vez e não é permitido interrupção de processamento. O objetivo é minimizar o makespan, quer dizer, minimizar o tempo de término de processamento 
da última operação no sistema.

O modelo usa variáveis binárias $x_{i k}\left(i=1, \ldots, o, k \in F_{i}\right)$ que indicam se a operação $i$ foi alocada para ser processada na máquina $k\left(x_{i k}=1\right)$ ou não $\left(x_{i k}=0\right)$. O modelo também utiliza variáveis binárias $y_{i j}\left(i, j=1 \ldots, o, F_{i} \cap F_{j} \neq \emptyset\right)$ para indicar, caso duas operações sejam alocadas para processamento numa mesma máquina, qual será processada primeiro. Por exemplo, se duas operações $i$ e $j$ que forem alocadas numa mesma máquina $k$, portanto $x_{i k}=x_{j k}=1$ e $k \in F_{i} \cap F_{j}$, significa que $y_{i j} \neq y_{j i}$ e consequentemente ou $y_{i j}=1$ ou $y_{j i}=1$. Se $y_{i j}=1$ e $y_{j i}=0$ então a operação $i$ é processada na máquina $k$ antes da operação $j$. Se $y_{j i}=1$ e $y_{i j}=0$ então a operação $i$ é processada na máquina $k$ depois do processamento da operação $j$. Por último, o modelo utiliza variáveis $s_{i}(i=1, \ldots, o)$ que representam o instante de início de processamento da operação $i$ na máquina em que ela foi alocada e a variável $C_{\max }$ para representar o makespan. Com tudo isso, o MIP para o FJSP-SF introduzido em Birgin et al. (2014) pode ser escrito como:

$$
\begin{aligned}
& \text { Minimize } \quad C_{\max } \\
& \text { Sujeito a: } \quad \sum_{k \in F_{i}} x_{i k}=1, \quad i=1, \ldots, o \text {, } \\
& p_{i}^{\prime}=\sum_{k \in F_{i}} x_{i k} p_{i k}, \quad i=1, \ldots, o, \\
& C_{\max } \geq s_{i}+p_{i}^{\prime}, \quad i=1, \ldots, o, \\
& s_{i}+p_{i}^{\prime} \leq s_{j}, \quad i, j=1, \ldots, o \text { tal que }(i, j) \in A, \\
& y_{i j}+y_{j i} \geq x_{i k}+x_{j k}-1, \quad i, j=1, \ldots, o, i \neq j, \text { e } k \in F_{i} \cap F_{j}, \\
& s_{i}+p_{i}^{\prime}-\left(1-y_{i j}\right) L \leq s_{j}, \quad i, j=1, \ldots, o \text { e } i \neq j \text { tal que } F_{i} \cap F_{j} \neq \emptyset \text {, } \\
& s_{i} \geq 0, \quad i=1, \ldots, o .
\end{aligned}
$$

A restrição (2.2) determina que cada operação $i$ só pode ser atribuída à somente uma máquina $k \in F_{i}$. A restrição (2.3) define o tempo de processamento $p_{i}^{\prime}$ da operação $i$, que depende da máquina em que a operação foi atribuída. O uso das variáveis $p_{i}^{\prime}$ somente simplifica a representação do modelo. A restrição (2.4) em conjunto com a minimização da função objetivo em (2.1) definem $C_{\max }$ como o makespan. A restrição (2.5) representa as restrições dadas pelo conjunto $A$. Para cada par de operações atribuídas à uma mesma máquina, as restrições (2.6) e (2.7) definem que duas operações não podem ser processadas ao mesmo tempo numa mesma máquina e determinam qual operação será processada primeiro. Além disso, se existe uma intersecção nos subconjuntos de máquinas de processamento de duas operações $i$ e $j$, isto é, se $F_{i} \cap F_{j} \neq \emptyset$ e contudo elas foram atribuídas à máquinas diferentes, implica que $x_{i k}+x_{j k} \leq 1$ e portanto as restrições (2.6) e (2.7) são satisfeitas trivialmente com $y_{i j}+y_{j i}=0$. Em $(2.7), L$ representa um número positivo suficientemente grande. Uma sugestão de valor para $L$ pode ser encontrada em Birgin et al. (2014). Finalmente, a restrição (2.8) determina que o instante de início de processamento das operações não deve ser menor que o início do horizonte de planejamento que, sem perda de generalidade, no MLPIM descrito, é 0 .

\subsection{Algoritmo List Scheduling}

Esta seção descreve o algoritmo list scheduling utilizado como base para a elaboração do método beam search posteriormente. Neste contexto, a Seção 2.2.1 introduz algumas características gerais que facilitarão o entendimento do método. Em seguida, a Seção 2.2.2 descreve como caracterizar uma solução parcial para o problema. Em seguida, a Seção 2.2.3 define as regras de seleção utilizadas para escolher sem ambiguidades qual operação será processada e em qual máquina isto ocorrerá. Para finalizar, a Seção 2.2.4 apresenta o pseudo-código do método, descrevendo suas principais partes. 


\subsubsection{Características gerais}

Será descrito a seguir um algoritmo list scheduling não-hierárquico. Ele é assim caracterizado devido que a cada iteração ele seleciona uma operação, atribui esta operação à uma máquina e define um instante de início de processamento. Este é um processo em contraste aos métodos hierárquicos que normalmente abordam o problema de programação em duas fases: numa primeira fase atribui operações às máquinas e em uma segunda fase determina os instantes de início de processamento de cada operação.

O método proposto itera $o$ vezes e, a cada iteração, seleciona uma operação $i$, atribui a uma máquina $k \in F_{i}$ e determina o instante de início de processamento $s t_{i k}$ da operação $i$ na máquina $k$ de forma construtiva. Neste processo, regras especializadas para o FJSP-SF guiam a seleção das operações e das máquinas. A decisão é gravada fazendo $s[i] \leftarrow s t_{i k}$ e $w[i] \leftarrow k$ que representam o instante de início de processamento da operação $i$ e a máquina $k$ que irá processar a operação $i$. Uma vez que os valores de $s[i]$ e $w[i]$ forem definidos no algoritmo, a operação $i$ será tratada como "manipulada" ou "programada". Dada uma iteração, as operações que são candidatas a serem programadas são aquelas que ainda não foram programadas e que possuem todas as suas operações precedentes programadas.

Os dados de entrada para o algoritmo são os parâmetros $n, m, F_{i}(i=1, \ldots, o), p_{i k}(i=$ $\left.1, \ldots, o, k \in F_{i}\right)$ e $A$, que caracterizam uma instância do FJSP-SF. A partir destes parâmetros, alguns dados auxiliares que ajudam a aplicação do método podem ser computados da seguinte maneira:

(a) Os conjuntos de predecessores e sucessores $\mathcal{P}_{i}$ e $\mathcal{S}_{i}$ dados por

$$
\mathcal{P}_{i}=\{j \in\{1, \ldots, o\} \mid(j, i) \in A\}
$$

$\mathrm{e}$

$$
\mathcal{S}_{i}=\{j \in\{1, \ldots, o\} \mid(i, j) \in A\} .
$$

(b) As médias dos tempos de processamento $\bar{p}_{i}$ dadas por

$$
\bar{p}_{i}=\frac{1}{\left|F_{i}\right|} \sum_{k \in F_{i}} p_{i k} .
$$

(c) O remaining work ou blocked work $R W_{i}$ dado pelo maior caminho partindo da operação $i$ até qualquer outra operação $j$ no DAG formado pelos nós nomeados de $\{1, \ldots, o\}$ com pesos $\bar{p}_{1}, \ldots, \bar{p}_{o}$ e arestas definidas em $A$.

\subsubsection{Soluções parciais}

A cada iteração, o list scheduling mantêm algumas informações que auxiliam a seleção da operação a ser programada, da máquina de processamento e do início de instante de processamento. São elas:

$s[i]$ O instante de início de processamento para cada operação $i$. Se a operação $i$ não foi programada, o valor armazenado é "não determinado".

$\boldsymbol{w}[\boldsymbol{i}]$ A máquina $k \in F_{i}$ que irá processar a operação $i$. Se a operação $i$ não foi programada, o valor armazenado é "não determinado".

$\boldsymbol{C}_{\max } \mathrm{O}$ valor máximo entre os instantes de término de processamento de todas as operações que já foram programadas, quer dizer, o makespan da solução parcial. 
$\boldsymbol{u}[\boldsymbol{i}]$ O valor máximo entre os instantes de término de processamento das operações que precedem $i$ e que já foram programadas.

$\boldsymbol{v}[\boldsymbol{k}]$ O valor máximo entre os instantes de término de processamento de todas as operações que já foram programadas e atribuídas a máquina $k$.

$\boldsymbol{L}[\boldsymbol{k}]$ A soma do tempo de processamento de todas as operações que ainda não foram programadas e que potencialmente poderiam ser atribuídas a máquina $k$. Este valor representa um limitante superior que estima a carga futura da máquina.

$\boldsymbol{\eta}[\boldsymbol{i}]$ A quantidade de operações predecessoras da operação $i$ que ainda não foram programadas.

$\boldsymbol{\Omega}$ Conjunto de operações candidatas a serem programadas, quer dizer, o conjunto de operações não programadas mas que possuem todas as suas precedentes programadas $(\eta[i]=0)$.

$\mathrm{Na}$ abordagem apresentada neste trabalho, uma solução parcial com $\ell$ operações programadas é caracterizada pela tupla $\left(\ell, s, w, C_{\max }, u, v, L, \eta, \Omega\right)$. Esta tupla armazena todas as informações que o algoritmo list scheduling, que será apresentado com mais detalhes na Seção 2.2.4, necessita para gerar uma nova solução parcial com $\ell+1$ operações programadas. Para o caso particular de uma solução parcial com $\ell=0$ operações programadas, a tupla é caracterizada como a seguir:

$$
\left\{\begin{array}{cl}
s[i] \quad & \text { não determinado para } i=1, \ldots, o, \\
w[i] & \text { não determinado para } i=1, \ldots, o, \\
C_{\max } & =0, \\
u[i] & =0 \text { for } i=1, \ldots, o, \\
v[k] & =0 \text { for } k=1, \ldots, m, \\
L[k] & =\sum_{\left\{i \mid k \in F_{i}\right\}} p_{i k} \text { para } k=1, \ldots, m, \\
\eta[i] & =\left|\mathcal{P}_{i}\right| \text { para } i=1, \ldots, o, \\
\Omega & =\left\{i \in\{1, \ldots, o\} \mid \mathcal{P}_{i}=\emptyset\right\} .
\end{array}\right.
$$

Dada uma solução parcial $\left(\ell, s, w, C_{\max }, u, v, L, \eta, \Omega\right)$ com $0 \leq \ell<o$, para escalonar uma operação $i \in \Omega$ para iniciar processamento no instante st na máquina $k \in F_{i}$, a nova tupla com $\ell+1$ operações programadas é $\left(\ell+1, s^{\prime}, w^{\prime}, C_{\max }^{\prime}, u^{\prime}, v^{\prime}, L^{\prime}, \eta^{\prime}, \Omega^{\prime}\right)$ onde $s^{\prime}, w^{\prime}, C_{\max }^{\prime}, u^{\prime}, v^{\prime}, L^{\prime}, \eta^{\prime}$ e $\Omega^{\prime}$ recebem, respectivamente, os valores de $s, w, C_{\max }, u, v, L, \eta$ e $\Omega$ e são atualizados como a seguir:

$$
\left\{\begin{aligned}
s^{\prime}[i] & \leftarrow s t, \\
w^{\prime}[i] & \leftarrow k, \\
C_{\max }^{\prime} & \leftarrow \max \left\{C_{\max }^{\prime}, s t+p_{i k}\right\}, \\
u^{\prime}[j] & \leftarrow \max \left\{u^{\prime}[j], s t+p_{i k}\right\} \text { para todo } j \in S_{i}, \\
v^{\prime}[k] & \leftarrow \max \left\{v^{\prime}[k], s t+p_{i k}\right\}, \\
L^{\prime}[r] & \leftarrow L^{\prime}[r]-p_{\text {ir }} \text { para todo } r \in F_{i}, \\
\eta^{\prime}[j] & \leftarrow \eta^{\prime}[j]-1 \text { para todo } j \in S_{i}, \\
\Omega^{\prime} & \leftarrow \Omega^{\prime} \backslash\{i\} \cup\left\{j \in S_{i} \mid \eta^{\prime}[j]=0\right\} .
\end{aligned}\right.
$$

\subsubsection{Regras de seleção}

Os critérios utilizados para selecionar a operação a ser programada, a máquina de processamento e o instante de início de processamento serão apresentados nesta seção. Nesta linha, inicialmente o 
list scheduling começa com uma solução parcial com $\ell=0$ operações programadas. A cada iteração, o algoritmo escalona uma única operação. Portanto, após $o$ iterações, o algoritmo termina toda a programação e com uma solução viável para a dada instância do FJSP-SF.

A cada iteração, o conjunto $\Psi_{0}$ de pares operação/máquina dado pelas operações possíveis de serem programadas e as suas respectivas máquinas de processamento é dado por

$$
\Psi_{0}=\left\{(i, k) \mid i \in \Omega \text { e } k \in F_{i}\right\} .
$$

A partir do conjunto $\Psi_{0}$, três regras são sequencialmente aplicadas até que um único par operação/máquina $(i, k) \in \Psi_{0}$ seja selecionado e um instante de início de processamento st seja determinado para a programação da operação $i$ na máquina $k$. As três regras são descritas a seguir:

Regra 1: Par operação/máquina com o menor instante de início de processamento possível.

O primeiro critério utilizado para selecionar um par $(i, k) \in \Psi_{0}$ é baseado no menor instante de processamento $s_{i k}$ possível para a operação $i$ na máquina $k$. Este valor pode ser facilmente calculado através do máximo entre dois valores: o máximo entre o instante de término de processamento de todas as operações que precedem a operação $i$, armazenado em $u[i]$ e o instante em que a máquina $k$ termina de processar todas as operações programadas e atribuídas à ela, armazenado em $v[k]$. Assim, calcula-se

$$
s t_{i k}=\max \{u[i], v[k]\}
$$

para todo $(i, k) \in \Psi_{0}$. Seja

$$
\widehat{s t}=\min \left\{s t_{i k} \mid(i, k) \in \Psi_{0}\right\}
$$

o menor instante de início de processamento possível entre todos os pares $(i, k) \in \Psi_{0}$ candidatos. Somente os pares $(i, k)$ tais que $s t_{i k}=\widehat{s t}$ permanecem como possibilidade para programação na iteração e os demais pares são descartados. Portanto, após aplicar a Regra 1, o conjunto de pares candidatos resultante $\Psi_{1}$ é dado por

$$
\Psi_{1}=\left\{(i, k) \mid(i, k) \in \Psi_{0} \text { e } s t_{i k}=\widehat{s t}\right\} .
$$

Regra 2: Máquina de processamento mais rápida.

Seja

$$
\Psi_{1}^{i}=\left\{(j, k) \mid(j, k) \in \Psi_{1} \text { e } j=i\right\} \text { for } i=1, \ldots, o,
$$

quer dizer, $\Psi_{1}^{i}$ é o subconjunto de pares operação/máquina em $\Psi_{1}$ fixando a operação $i$. O objetivo nesta regra é determinar uma única máquina de processamento para as operações $i$ que possuem várias máquinas de processamento como possibilidade, quer dizer, para todo $\Psi_{1}^{i} \operatorname{com} i=1, \ldots, o$ e $\left|\Psi_{1}^{i}\right|>1$, objetiva-se reduzir a cardinalidade para $\left|\Psi_{1}^{i}\right|=1$ escolhendo uma única máquina dentre todas as possíveis.

Seja a operação $i$ tal que $\left|\Psi_{1}^{i}\right|>1$ e sejam os pares $\left(i, k_{1}\right),\left(i, k_{2}\right), \ldots$ os elementos de $\Psi_{1}^{i}$. Cada máquina $k_{1}, k_{2}, \ldots$ está associada a um tempo de processamento $p_{i k_{1}}, p_{i k_{2}}, \ldots$ e a um limitante superior da carga na máquina $L\left[k_{1}\right], L\left[k_{2}\right], \ldots$ Considere as triplas

$$
\left(p_{i k_{1}}, L\left[k_{1}\right], k_{1}\right),\left(p_{i k_{2}}, L\left[k_{2}\right], k_{2}\right), \ldots
$$

Seja o conjunto $\Psi_{1.5}^{i}=\left\{\left(i, k_{\nu}\right)\right\}$ formado por um único par tal que $\left(p_{i k_{v}}, L\left[k_{v}\right], k_{v}\right)$ é a menor tripla da ordem lexicográfica destas triplas. Isto significa que, dentre todas as possíveis máquinas para processar a operação $i$, seleciona-se aquela com o menor tempo de processamento (mais rápida). Caso houver empates, a máquina com o menor valor de limitante superior de carga associada à 
ela é preferida. Caso ainda persista algum empate, a máquina com o menor índice é escolhida com propósito único de definir uma regra determinística. Portanto, após aplicar a Regra 2 no conjunto $\Psi_{1}$, o conjunto $\Psi_{2}$ resultante é dado por

$$
\Psi_{2}=\left\{\cup \Psi_{1}^{i}|| \Psi_{1}^{i} \mid \leq 1\right\} \cup\left\{\cup \Psi_{1.5}^{i}|| \Psi_{1}^{i} \mid>1\right\},
$$

que consiste no subconjunto de $\Psi_{1}$ que contém somente um par de operação/máquina para cada operação. Nos casos onde haviam mais de uma máquina de processamento possível, preserva-se somente os pares associados às máquinas mais rápidas e com a menor estimativa de carga futura.

\section{Regra 3: Operação com o maior remaining (blocked) work.}

O próximo atributo utilizado para reduzir a quantidade de pares candidatos $(i, k) \in \Psi_{2}$ é baseado na estimativa do remaining (blocked) work $R W_{i}$ associado com a operação $i$. Lembre-se que $R W_{i}$ é definido como o maior caminho partindo da operação $i$ para qualquer outra operação $j$ no DAG com conjunto de arcos $A$, conjunto de nós $\{1, \ldots, o\}$ com pesos $\bar{p}_{1}, \ldots, \bar{p}_{o}$. Note que se o maior caminho começa na operação $i$ e termina em uma operação $j$ então $\mathcal{S}_{j}=\emptyset$ e $i$ e $j$ pertencem à uma mesma tarefa $t$. Além disso, $s t_{i k}+R W_{i}$ pode ser visto, na perspectiva do par $(i, k) \in \Psi_{2}$, como uma estimativa do instante de tempo em que a tarefa $t$ irá terminar de ser processada. Contudo, uma vez que $s t_{i k}=\widehat{s t}$ para todo $(i, k) \in \Psi_{2}$ por definição, então considera-se simplesmente o valor de $R W_{i}$ como a estimativa do remaining work associado com a operação $i$.

Para os pares $\left(i_{1}, k_{1}\right),\left(i_{2}, k_{2}\right), \cdots \in \Psi_{2}$ considere as triplas

$$
\left(-R W_{i_{1}},-L\left[k_{1}\right], i_{1}\right),\left(-R W_{i_{2}},-L\left[k_{2}\right], i_{2}\right), \ldots
$$

e seja $\left(-R W_{i_{v}},-L\left[k_{v}\right], i_{v}\right)$ a menor tripla considerando a ordem lexicográfica associada com o par $\left(i_{v}, k_{v}\right)$. Isto significa que a última escolha foi feita e que ela consiste em atribuir a operação $i_{v}$ à máquina $k_{v}$ com instante de início de processamento $\widehat{s t}$. Nesta linha, a operação com o maior remaining work foi selecionada para ser programada. A ideia por trás desta escolha é escalonar rapidamente as operações que emperram o processamento de uma grande quantidade de trabalho. No caso de empate, o limitante superior da carga futura das máquinas é utilizado como critério de desempate, visando minimizar o tempo ocioso de máquinas que tem uma alta carga futura, atribuindo operações o mais cedo possível à elas. Finalmente, no caso de um segundo empate, a operação com o menor índice é escolhida com propósito único de definir uma regra de escolha determinística.

\subsubsection{Pseudo-código}

O algoritmo list scheduling, inteiramente descrito pelo Algoritmo 1, é introduzido nesta seção. Para isto, a inicialização da solução vazia $(\ell=0)$ definida na Seção 2.2.2, a aplicação iterativa das Regras 1-3 da Seção 2.2.3 e a atualização da solução parcial descrita também na Seção 2.2.2 a partir do par operação/máquina escolhido serão utilizados em harmonia e definirão o método proposto.

No Algoritmo 1, os conjuntos $\mathcal{P}_{i}$ e $\mathcal{S}_{i}$ são computados nas linhas 2-3. As médias dos tempos de processamento $\bar{p}_{i}$ são computadas nas linhas 4-7. A estimativa do remaining work $R W_{i}$ são feitas nas linhas 8-16 pelo método de Dijkstra de menor caminho (Cormen et al., 2009) adaptado para calcular o maior caminho no ambiente de várias origens e vários destinos, que é o caso do DAG do FJSP-SF. Os valores de $C_{\max }, u, v, L, \eta$, e o conjunto $\Omega$ são inicializados nas linhas 17-21. O laço principal, da linha 22 até a linha 41, executa $o$ vezes as Regras 1-3. O par operação/máquina de cada iteração é denominado $(\lambda, \theta)$ e o instante de início de processamento determinado é nomeado $\widehat{s t}$. O instante de início de processamento $s[\lambda]$ para a operação $\lambda$ é atribuído na linha 36 assim como a atribuição da máquina $\theta$ para processar a operação $\lambda$, definindo $w[\lambda] \operatorname{como} \theta$. Os valores de $C_{\max }$, $u, v, L, \eta$, e do conjunto $\Omega$ são atualizados nas linhas 36-41. 




\subsubsection{Exemplo}

Para entender melhor o funcionamento do list scheduling proposto nesta seção, considere o cenário dado pela Figura 2.3. De acordo com a figura, o conjunto $\Psi_{0}$ é formado pelos pares $\left(o_{1}, m_{1}\right),\left(o_{1}, m_{2}\right),\left(o_{5}, m_{1}\right),\left(o_{5}, m_{2}\right)$ e $\left(o_{6}, m_{2}\right)$. Esses pares são compostos pelas operações cujas operações precedentes já foram programadas, quer dizer, não possuem precedentes pendentes, em conjunto com as máquinas que possam processá-las. A Figura 2.4 apresenta estas opções em um 
formato de árvore. Na figura, o primeiro nó da árvore representa o início do horizonte de processamento, onde nada ainda foi escolhido para ser programado. O segundo nível da árvore são as opções de pares operação/máquinas representados por $\Psi_{0}$.



Figura 2.3: DAG de precedências e tabela de tempos de processamento de um cenário do FJSP-SF com $o=8$ operações e $m=2$ máquinas.



Figura 2.4: Pares operação/máquinas possíveis de serem programados considerando o cenário inicial da Figura 2.3.

Ao aplicarmos a Regra 1 no conjunto $\Psi_{0}$, todos os pares operação/máquina são selecionados para o conjunto $\Psi_{1}$. Isso acontece pois nenhuma operação foi selecionada ainda para ser programada e portanto, todas as máquinas estão livres no início do horizonte de processamento implicando em todas as operações disponíveis poderem iniciar processamento no mesmo instante. A Figura 2.5 ilustra a árvore de busca dada por $\Psi_{1}$.

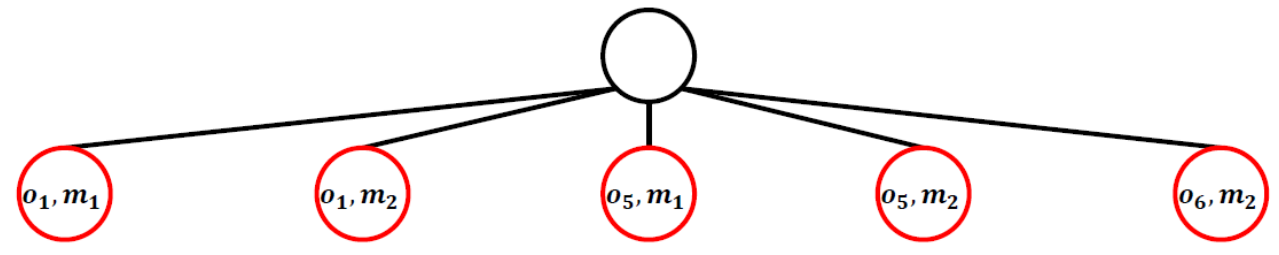

Figura 2.5: Conjunto de pares operação/máquina após a Regra 1 ser aplicada ao cenário descrito pela Figura 2.3.

Agora é a vez da Regra 2 ser aplicada sobre o conjunto $\Psi_{1}$. Neste caso, deseja-se selecionar as máquinas mais rápidas possíveis para cada uma das operações disponíveis e em caso de empates, a máquina com o menor índice será escolhida. A Figura 2.6 ilustra este processo. Na figura, os pares $\left(o_{1}, m_{1}\right),\left(o_{5}, m_{2}\right)$ e $\left(o_{6}, m_{2}\right)$ são os pares selecionados para compor o conjunto $\Psi_{2}$. A operação $o_{1}$ possuía duas máquinas possíveis para ser processadas. Ambas as máquinas poderiam processar a operação em 4 instantes de tempo. A máquina $m_{1}$ foi selecionada pela regra de menor índice. A operação $o_{5}$ também poderia ser processada pela máquina $m_{1}$ e pela máquina $m_{2}$. A máquina 
$m_{2}$ foi selecionada pois processa a operação em menos tempo, quer dizer, mais rapidamente. Por último, a máquina $m_{2}$ foi selecionada para a operação $o_{6}$ pois é a única máquina que pode processar a operação.

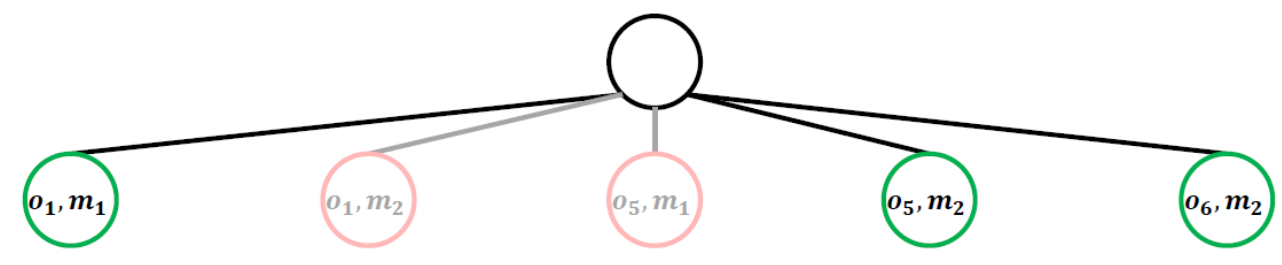

Figura 2.6: Conjunto de pares operação/máquina após a Regra 1 e a Regra 2 serem aplicadas ao cenário descrito pela Figura 2.3.

Por último, a Regra 3 é aplicada sobre o conjunto $\Psi_{2}$. Nesta regra, deseja-se escolher o par operação/máquina vinculado ao maior remaining work. $\mathrm{O}$ valor de $R W_{i}$ é mostrado na Figura 2.3 para cada operação. De acordo com os valores, a operação $o_{1}$ é a operação com o maior valor $R W_{1}=10$ e portanto, o par $\left(o_{1}, m_{1}\right)$ é o o primeiro par selecionado a ser programado. A Figura 2.7 apresenta o gráfico de Gant com o par $\left(o_{1}, m_{1}\right)$ programado. A Figura 2.8 ilustra a árvore de busca com as novas operações possíveis de serem programadas.

\begin{tabular}{|c|c|}
\hline$M_{2}$ & \\
\hline$M_{1}$ & $o_{1}$ \\
\hline
\end{tabular}

Figura 2.7: Gráfico de Gant da programação da operação o $o_{1}$ na máquina $m_{1}$ considerando o cenário descrito pela Figura 2.3.

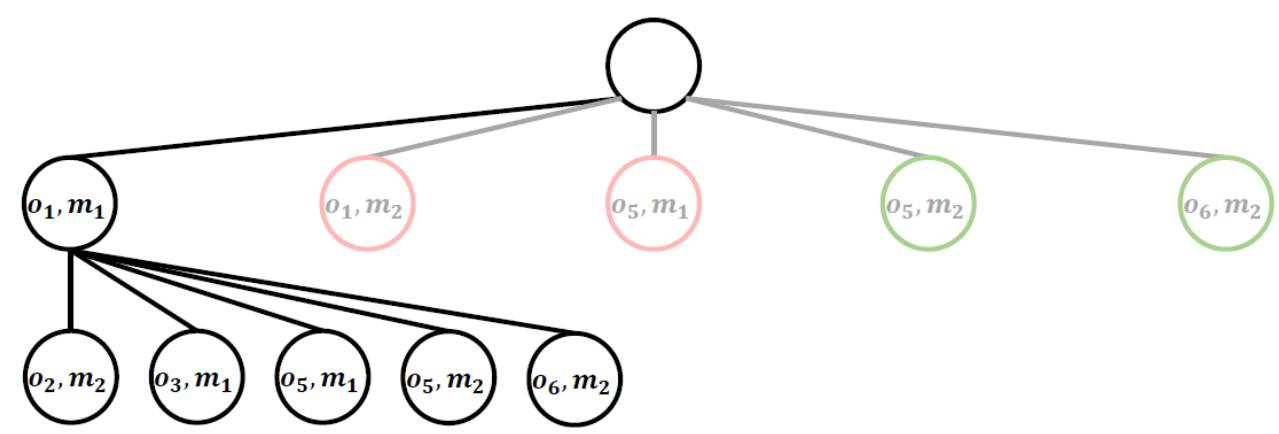

Figura 2.8: Árvore de busca após as Regras 1-3 serem aplicadas no cenário descrito pela Figura 2.3 e a programação do par $\left(o_{1}, m_{1}\right)$.

Portanto, ao aplicarmos as Regras 1-3 o vezes em um cenário qualquer do FJSP-SF, o pares operação/máquinas serão escolhidos e uma programação completa poderá ser feita. Este processo descreve o algoritmo list scheduling proposto nesta seção.

\subsection{Método Beam Search}

O algoritmo list scheduling descrito na Seção 2.2, a cada iteração, seleciona uma única operação, atribui a uma máquina e determina o instante de início de processamento desta operação. Cada uma destas decisões é feita utilizando regras heurísticas. Mesmo que estas decisões fossem decisões 
ótimas, por se tratarem de decisões locais, o algoritmo como um todo não geraria necessariamente uma programação de boa qualidade. Assim, com custo de aumentar a complexidade do algoritmo, faz sentido lidar com a construção de mais de uma programação por vez, selecionando e analisando não somente uma operação por iteração mas sim um pequeno conjunto de operações. Nesta linha, uma solução parcial com $\ell$ operações programadas pode originar várias outras soluções parciais com $\ell+1$ operações programadas desde que diferentes operações sejam manipuladas. Esta descrição encaixa com a descrição da árvore de busca de um método Beam Search. Portanto, é possível dizer que o método Beam Search construído e apresentado nesta seção é uma extensão natural do método list scheduling da seção anterior.

O método Beam Search deste trabalho é um Filtered Beam Search que mantém aproximadamente $f(\beta)$ nós em cada nível da árvore de busca onde $\beta \in(0,1]$ é um parâmetro e $f: \mathbb{R} \rightarrow \mathbb{N}$ é uma função que será determinada posteriormente. Em um dado nível $\ell$ da árvore, cada nó representa uma solução parcial com $\ell$ operações programadas. Utilizando as Regras 1-3 descritas na Seção 2.2.3 é possível selecionar um pequeno conjunto de $g(\alpha)$ operações mais promissoras que poderiam ser programadas, onde $\alpha \in(0,1]$ é um parâmetro do método e $g: \mathbb{R} \rightarrow \mathbb{N}$. No contexto do método Beam Search, este passo é feito com a avaliação local, que é uma avaliação simplificada dos nós e que consome pouco tempo computacional. Portanto, uma solução parcial com $\ell$ operações origina $g(\alpha)$ outras soluções parciais com $\ell+1$ operações programadas. A partir daí, a avaliação global, que é mais cara computacionalmente, é responsável por escolher uma das $g(\alpha)$ soluções parciais. No método apresentado nesta seção, a avaliação global consiste em completar cada solução parcial utilizando o algoritmo list scheduling apresentado na seção anterior e a partir do makespan obtido, selecionar a melhor solução parcial, dentre as $g(\alpha)$, para o próximo nível da árvore. Neste trabalho, a estratégia de manter apenas um filho por nó sugerida em Sabuncuoglu e Bayiz (1999) foi adotada.

\subsubsection{Estratégias de avaliação local e global}

Seja uma solução parcial $\left(\ell, s, w, u, v, L, C_{\max }, \eta, \Omega\right) \operatorname{com} \ell<o$ ou de maneira equivalente, seja um nó do nível $\ell$ da árvore de busca. É possível aplicar as Regras 1-3 da Seção 2.2.3 nesta solução de maneira a selecionar o par operação/máquina $\left(i_{1}, k_{1}\right) \in \Psi_{0}$ e designar um instante de início de processamento $s t_{i_{1} k_{1}}$ e a partir de tudo isso, gerar uma nova solução parcial, ou em termos de árvore de busca: um filho, com $\ell+1$ operações programadas utilizando a estratégia descrita na Seção 2.2.2. Pode-se dizer que do ponto de vista de uma estratégia local utilizando as Regras 1-3, o filho gerado consiste no filho mais promissor possível. Considere agora que o par $\left(i_{1}, k_{1}\right)$ é proibido. Se for aplicado as Regras 1-3 novamente ao mesmo nó $\left(\ell, s, w, u, v, L, C_{\max }, \eta, \Omega\right)$, um novo par operação/máquina $\left(i_{2}, k_{2}\right) \in \Psi_{0}$ será obtido e um novo instante de início de processamento $s t_{i_{2} k_{2}}$ pode ser designado. A partir do mesmo nó, pode-se gerar um novo filho com $\ell+1$ operações programadas utilizando o novo par $\left(i_{2}, k_{2}\right) \in \Psi_{0}$. Pode-se dizer também que este segundo filho é o segundo mais promissor do ponto de vista da mesma estratégia local.

É possível gerar vários filhos a partir do que foi descrito no parágrafo anterior. Contudo, visto que a estratégia global consiste em uma estratégia computacionalmente mais cara quando comparada a estratégia local, aplicar a estratégia global em todos os possíveis filhos gerados seria inviável. Assim, no método proposto, um limite para a quantidade de filhos gerados é imposto de forma a restringir a quantidade de vezes que a estratégia global será utilizada. Este limite é uma função do parâmetro $\alpha \in(0,1]$ que idealmente deveria ser independente do tamanho da instância. Porém, com o intuito de não comprometer o desempenho do método em instâncias de grande porte, o limite dado por $\hat{\alpha_{1}} \equiv \alpha\left\lceil\Psi_{0}\right\rceil$ é adotado. É importante notar que $\hat{\alpha_{1}}$ varia de acordo com o nó em questão visto que $\Psi_{0}$, como definido em (2.9), varia de acordo com o conjunto $\Omega$. Além do limite $\hat{\alpha_{1}}$, um segundo limite $\hat{\alpha_{2}}$ que visa preservar a qualidade dos filhos gerados também é adotado. Para entender este limite, primeiramente note que ao aplicar as Regras 1-3 em um dado nó $\left(\ell, s, w, u, v, L, C_{\max }, \eta, \Omega\right)$ obtêm-se o par operação/máquina $\left(i_{1}, k_{1}\right)$ com instante de início de processamento $s t_{i_{1} k_{1}}$. Ao aplicar as Regras 1-3 novamente no mesmo nó, proibindo o par $\left(i_{1}, k_{1}\right)$, obtêm-se o par operação/máquina $\left(i_{2}, k_{2}\right)$ com instante de início de processamento $s t_{i_{2} k_{2}}$. Contudo, $s t_{i_{1} k_{1}} \leq s t_{i_{2} k_{2}}$ e portanto o par $\left(i_{2}, k_{2}\right)$ não necessariamente é um par com o menor instante de 
início de processamento possível, o que de certa forma, quebra o intuito da Regra 1. Assim, para garantir um limite máximo no instante de início de processamento dos pares selecionados, define-se o conjunto

$$
\Psi_{0.5}=\left\{(i, k) \mid(i, k) \in \Psi_{0} \text { e } s t_{i k} \leq \widehat{s t}+\xi \widehat{p}\right\} \subseteq \Psi_{0},
$$

onde

$$
\widehat{s t}=\min \left\{s t_{i k} \mid(i, k) \in \Psi_{0}\right\} \text { e } \widehat{p}=\max \left\{p_{i k} \mid(i, k) \in \Psi_{0}\right\}
$$

e define-se o limite $\hat{\alpha}_{2} \equiv\left|\Psi_{0.5}\right|$. Desta forma, o limite máximo do instante de início de processamento dos filhos gerados não pode ser maior que $\widehat{s t}+\xi \widehat{p}$, onde $\xi$ é outro parâmetro do método. Finalmente, têm-se que a quantidade máxima de filhos selecionados pela estratégia local a partir de um dado nó na árvore de busca é limitada por $\hat{\alpha}=\min \left\{\hat{\alpha_{1}}, \hat{\alpha_{2}}\right\}$, o que define a função $g(\alpha)$.

A estratégia global adotada neste trabalho consiste em aplicar o algoritmo list scheduling descrito na Seção 2.2 no pequeno conjunto de filhos gerados a partir da estratégia local descrita no parágrafo anterior completando cada programação parcial. A partir de cada programação completa, é possível computar um makespan $C_{\max }^{\text {est }}$ associado com a programação. Finalmente, seleciona-se para a próxima geração da árvore somente o filho do pequeno conjunto com o menor $C_{\max }^{\text {est }}$ obtido completando a programação parcial e todos os outros filhos são "cortados" da árvore de busca. É importante notar que o limite $\hat{\alpha}$ "filtra" a quantidade de vezes que o algoritmo list scheduling será utilizado uma vez que o algoritmo não é aplicado em todos os possíveis filhos de um nó da árvore.

Na próxima seção, o pseudo-código do método Beam Search será apresentado e os detalhes técnicos dos critérios de desempate entre os filhos bem como alguns outros detalhes menores serão descritos.

\subsubsection{Pseudo-código}

Os Algoritmos 2-5 apresentam inteiramente e descrevem com detalhes o método Beam Search proposto neste trabalho. O Algoritmo 2 descreve a rotina BEAMSEARCHIni que faz as inicializações necessárias para o método e constrói o primeiro nível da árvore de busca que é um pouco diferente da construção dos demais níveis. O Algoritmo 3 descreve a rotina CMAXEsT que estima o makespan de uma dada solução parcial completando a sua programação através de uma re-implementação do Algoritmo 1 apresentado na Seção 2.2. Esta re-implementação remove as inicializações e adapta o algoritmo para receber uma solução parcial. O Algoritmo 4 descreve a rotina SELECT que recebe uma solução parcial e seleciona um par operação/máquina que pode ser utilizado para expandir esta solução. Em linhas gerais, as rotinas SELECT e CMAXEsT desempenham o papel das avaliações local e global.

O Algoritmo 2 recebe como entrada $\left(n, m, o, F_{i}(i=1, \ldots, o), p_{i k}\left(i=1, \ldots, o, k \in F_{i}\right)\right.$, $A)$, que são dados que caracterizam uma instância do FJSP-SF, e os valores escalares $\xi>0$, $\alpha, \beta \in(0,1]$. O parâmetro $\xi$ diz respeito a tolerância máxima pelo quanto o instante de início de processamento de um par operação/máquina $(i, k) \in \Psi_{0}$ pode ser superior ao menor instante de início de processamento possível $\widehat{s t}$ entre todos os pares em $\Psi_{0}$, como definido em $(2.13,2.14)$. O parâmetro $\alpha$ está relacionado a quantidade de nós que a estratégia local seleciona para que a busca global seja aplicada. O parâmetro $\beta$ determina a quantidade de nós abertos por nível na árvore.

As linhas 2-16 do Algoritmo 2 inicializam $\mathcal{P}_{i}, \mathcal{S}_{i}, \bar{p}_{i}$, e $R W_{i}(i=1, \ldots, o)$ da mesma maneira que o Algoritmo 1. As linhas 17-21 inicializam $C_{\max }^{\prime}, u^{\prime}, v^{\prime}, L^{\prime}, \eta^{\prime}$ e o conjunto $\Omega^{\prime}$ que estão associados ao nó raiz da árvore, que representa uma solução parcial com nenhuma operação programada. No primeiro nível, as soluções parciais são formadas por apenas uma única operação programada e a qualidade dos nós mais promissores escolhidos impacta diretamente no desempenho do método. Além disso, a ramificação deste nível é feita apenas a partir do nó raiz e o máximo de filhos possíveis do nó raiz é dado por $\mathrm{nm}$. Visto tudo isso, nenhum filtro é utilizado na construção do primeiro nível da árvore de busca e todos os pares operação/máquina são avaliados pela estratégia global que por sua vez seleciona os nós mais promissores para comporem o primeiro nível. As linhas 22-34 consideram cada par operação/máquina, como definido em (2.9), constroem a respectiva solução parcial, estimam o makespan através da busca global da linha 33 e salva a solução parcial com o 
respectivo makespan estimado no conjunto $\mathcal{N}^{\prime}$. Nas linhas 35 e 36, a fração $\beta$ das soluções parciais mais promissoras de $\mathcal{N}^{\prime}$ são copiadas em $\mathcal{N}$ que por sua vez constitui de fato o primeiro nível da árvore. É importante notar que, em caso de empates, a cardinalidade de $\mathcal{N}$ pode ser maior que $\hat{\beta} \equiv\left\lceil\beta\left|\mathcal{N}^{\prime}\right|\right\rceil$. Finalmente, na linha 37, a rotina BEAMSEARCH recebe o primeiro nível, constrói todo o restante da árvore e retorna a melhor folha encontrada.

O Algoritmo 5 descreve a rotina BEAMSEARCH que itera $o-1$ vezes e cada iteração inicia com o conjunto $\mathcal{N}$ composto pelos nós do nível atual da árvore de busca. Estes nós são temporariamente rotulados de $1, \ldots,|\mathcal{N}|$ e esse rótulo é guardado na variável nid. Para cada nó, um conjunto de filhos denominado $\mathcal{D}_{\text {nid }}$ é construído. As linhas 6-16 implementam o limite de filhos para cada nó, como discutido na Seção 2.3.1. Posteriormente, um único filho para cada nó é selecionado e copiado no conjunto $\mathcal{N}^{\prime}$. A iteração termina substituindo $\mathcal{N}$ por $\mathcal{N}^{\prime}$. É importante notar que somente os nós do nível atual da árvore são necessários para o método visto que cada nó carrega toda a informação da representação da solução parcial. Dessa forma, nenhuma estrutura de árvore é de fato implementada.

Os filhos de um dado nó são construídos nas linhas 17-28 e salvos no conjunto $\mathcal{D}_{\text {nid }}$, que é inicializado como um conjunto vazio na linha 17 . Um total de $\min \left\{\hat{\alpha}_{1}, \hat{\alpha}_{2}\right\}$ filhos são gerados, onde $\hat{\alpha}_{1}$ é computado na linha 6 e $\hat{\alpha}_{2}$ é computado nas linhas 12-16. Na linha 19, uma cópia $\left(s^{\prime}, w^{\prime}, C_{\max }^{\prime}, u^{\prime}, v^{\prime}, L^{\prime}, \eta^{\prime}, \Omega^{\prime}\right)$ da solução $\left(s, w, C_{\max }, u, v, L, \eta, \Omega\right)$ é feita. Na linha 20, um par operação/máquina $(\lambda, \theta)$ é selecionado através da rotina SELECT. Nas linhas 21-26, o par $(\lambda, \theta)$ é escalonado e uma nova solução parcial é construída. A chamada da rotina CMAXEsT na linha 27 estima o valor do makespan $C_{\max }^{\text {est }}$ da nova solução parcial. Finalmente, a solução parcial com $(\lambda, \theta)$ já escalonados é colocada no conjunto $\mathcal{D}_{\text {nid }}$, juntamente com o par $(\lambda, \theta)$ e o valor de $C_{\text {max }}^{\text {est }}$. Para que nas próximas chamadas da rotina SELECT um par diferente de $(\lambda, \theta)$ seja devolvido e consequentemente um filho diferente seja gerado, o par $(\lambda, \theta)$ é adicionado ao conjunto $\mathcal{F}$. Todos os pares no conjunto $\mathcal{F}$ são tidos como proibidos nas próximas chamadas de SELECT. Dessa forma, é importante notar que a rotina SELECT implementa as Regras 1-3 com a única diferença que os pares são escolhidos a partir de $\Psi_{0} \backslash \mathcal{F}$.

Portanto, cada nó do nível possui um conjunto de filhos, quer dizer, cada nó está vinculado a um nid que por sua vez está vinculado a um conjunto de filhos $\mathcal{D}_{\text {nid }}$. Contudo, apenas um filho de cada conjunto deve ser escolhido para o próximo nível da árvore. Esta escolha é feita baseada no valor do $C_{\max }^{\text {est }}$ de cada solução parcial e utiliza os pares $(\lambda, \theta)$ como critério de desempate. Além da tarefa de simplificar cada conjunto de filhos a um único filho por nó, existe outra tarefa que requer inspecionar todos os conjuntos $\mathcal{D}_{\text {nid }}$ simultaneamente: evitar filhos idênticos de diferentes soluções parciais em $\mathcal{N}$. Note que no primeiro nível da árvore de busca, todos os nós são obrigatoriamente diferentes visto que consistem em soluções parciais oriundas de uma única operação programada a partir de pares operação/máquina diferentes. Contudo, duas diferentes soluções parciais quando expandidas podem se tornar idênticas. Além disso, se em algum nível da árvore de busca existem soluções idênticas, as expansões dessas soluções serão idênticas até as folhas e portanto, gerarão soluções completas idênticas, afetando diretamente e de maneira negativa a diversidade do método. Para evitar esta situação, antes de escolher um único filho por nó, soluções parciais idênticas são removidas da $\cup \mathcal{D}_{\text {nid }}$. Caso soluções parciais idênticas existam, a solução com o menor $\lambda$ é preservada e em caso de empate, a solução com o menor $\theta$. Esta eliminação de soluções parciais idênticas é feita no laço da linha 30 , onde os valores de $C_{\max }^{\text {est }}$ destas soluções são marcados como $\infty$. Na linha 37 as soluções marcadas são de fato removidas. Finalmente, o melhor filho de cada pai é escolhido nas linhas 38 e 39 e copiados no conjunto $\mathcal{N}^{\prime}$ que se torna o conjunto de nós $\mathcal{N}$ na linha 40, no final da iteração do laço principal. 


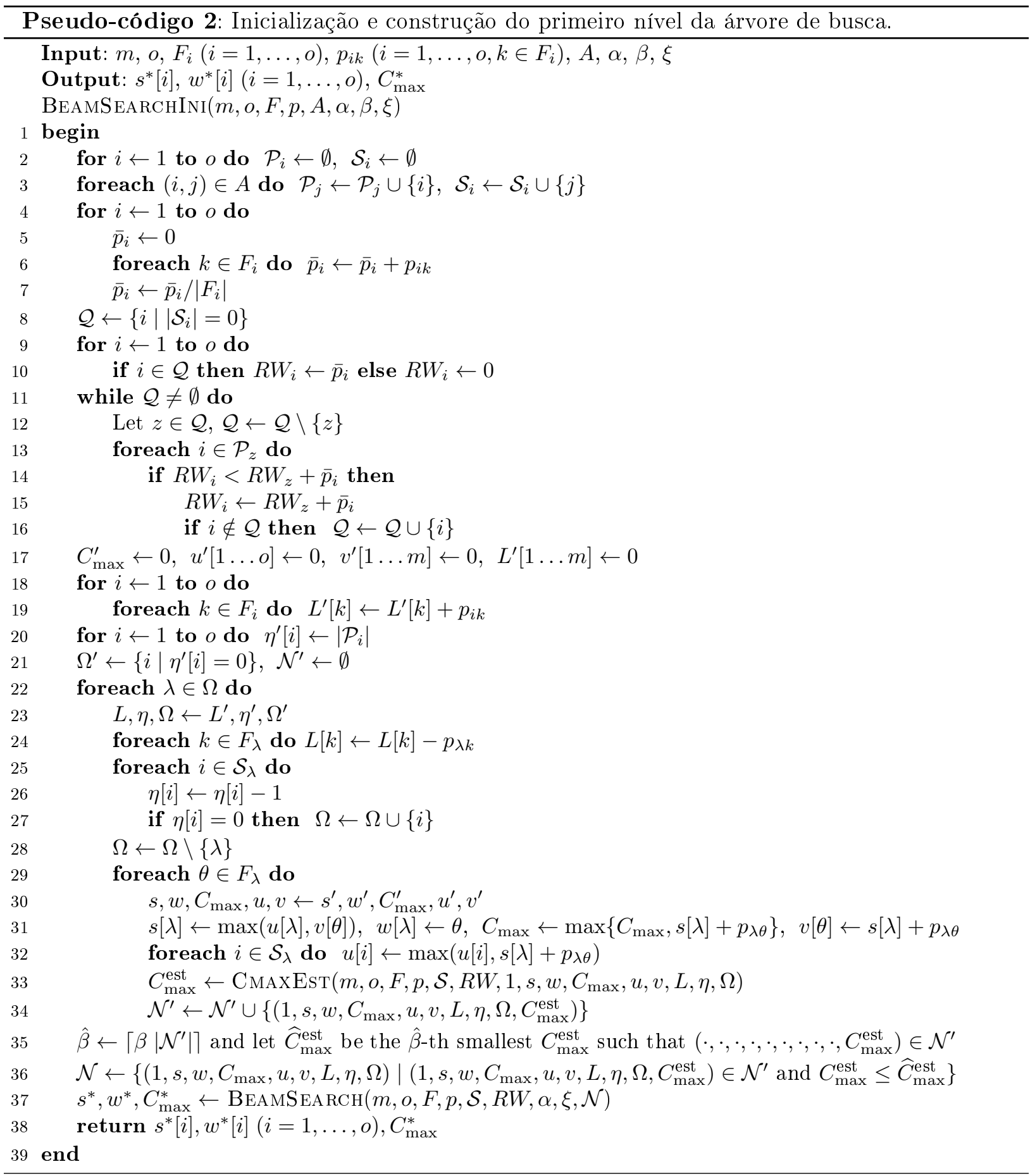



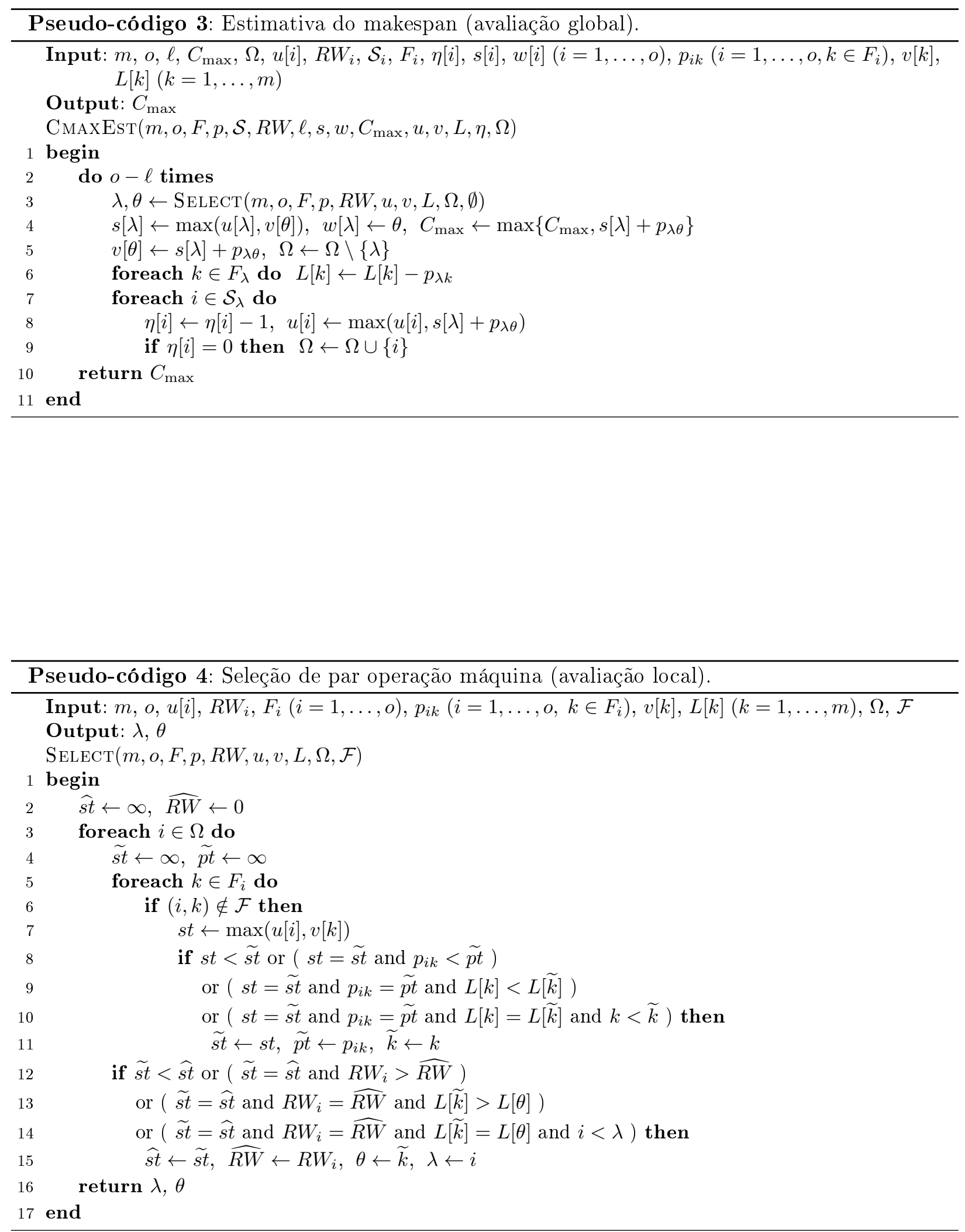







\subsection{Experimentos Numéricos}

O algoritmo list scheduling e o método Beam Search, Algoritmos 1 e 2-5, foram implementados em $\mathrm{C} / \mathrm{C}++$ e os códigos fonte estão disponíveis em http://www.ime.usp.br/ egbirgin/. A compilação dos códigos foi feita utilizando o compilador g ++ do GCC, versão 4.7.2 Debian 4.7.2-5, utilizando a opção de otimização "-O3". Todos os testes foram feitos utilizando um computador Intel(R) Xeon(R) E5645 2.40GHz com 132 GB de memória RAM e executados no sistema operacional Debian 7, kernel 3.7.6 SMP x86_64. A descrição detalhada dos resultados numéricos em conjunto com a descrição completa das soluções obtidas também estão disponibilizadas com os códigos fontes.

Para poder avaliar o comportamento dos métodos propostos quando aplicados em instâncias de pequeno e médio porte, um solver exato foi aplicado em 50 instâncias do modelo (2.1-2.8) introduzidas em Birgin et al. (2014). Estas instâncias são divididas em dois grupos de 20 e 30 instâncias, denominados de YFJS e instâncias DAFJS respectivamente. As instâncias YFJS01YFJS20 são instâncias compostas por duas sequências de operações seguidas por uma operação de montagem que une os dois componentes já processados, denominados como Y-jobs em Birgin et al. (2014). As instâncias DAFJS01-DAFJS30 são instâncias compostas por tarefas cujas as precedências são dadas por um DAG arbitrário. As Tabelas 2.1 e 2.2 descrevem as principais características de cada instância. As Figuras 2.9 e 2.10 ilustram os tipos de precedências das operações de cada um dos conjuntos de instâncias, quer dizer, ilustram a flexibilidade de sequenciamento de cada um dos conjuntos. Como solver exato foi utilizado o IBM ILOG CPLEX $12.1 \mathrm{com}$ as seguintes configurações: 1 para o número máximo de threads e 2048MB de memória disponível. Todos os demais parâmetros foram deixados como o padrão. Duas baterias de testes com o solver foram feitas. Na primeira, o tempo de CPU foi de 1 hora. Na segunda, foram consideradas somente as instâncias nas quais as soluções ótimas não foram encontradas e foi dado 10 horas de tempo de CPU. As Tabelas 2.3 e 2.4 apresentam os resultados para os conjuntos de instâncias YFJS01-YFJS20 e DAFJS01-DAFJS30, respectivamente. Nas tabelas, "mks" é o makespan e "CPU(s)" é o tempo de CPU em segundos. Nos casos onde o solver atingiu o limite de tempo sem encontrar uma solução ótima, a tabela apresenta o limitante inferior obtido e o gap relativo, dado pela diferença entre os limitantes dividido pelo limitante superior. Na Tabela 2.3 o solver conseguiu encontrar soluções ótimas para 14 (YFJS01-YFJS14) das 20 instâncias do conjunto e também um gap menor que $1 \%$ foi obtido em 2 outras instâncias (YFJS15 e YFJS16). Por outro lado, a Tabela 2.4 mostra que o solver encontrou soluções ótimas em 4 das 30 instâncias e gap menor que $1 \%$ em apenas 1 instância, quando o conjunto DAFJS01-DAFJS30 foi considerado. Estes resultados também ajudam a explicar as "melhorias" obtidas pelos métodos heurísticos propostos neste trabalho quando comparados às soluções obtidas pelo solver com limite de tempo de CPU.



Figura 2.9: Representação gráfica das precedências entre as operações da instância YFJS02. 


\begin{tabular}{|c|cc|ccc|ccc|ccc|}
\hline \multirow{2}{*}{ Nome } & \multirow{2}{*}{$n$} & \multirow{2}{*}{$m$} & \multicolumn{3}{|c|}{ \# de operações } & \multicolumn{3}{|c|}{$\left|F_{i}\right|$} & \multicolumn{3}{c|}{ de precedências } \\
\cline { 4 - 11 } & & & $\min$ & $\max$ & $\sum$ & $\min$ & $\max$ & $\sum$ & $\min$ & $\max$ & $\sum$ \\
\hline \hline YFJS01 & 4 & 7 & 10 & 10 & 40 & 1 & 3 & 104 & 0 & 2 & 36 \\
YFJS02 & 4 & 7 & 10 & 10 & 40 & 1 & 3 & 104 & 0 & 2 & 36 \\
YFJS03 & 6 & 7 & 4 & 4 & 24 & 2 & 3 & 63 & 0 & 2 & 18 \\
YFJS04 & 7 & 7 & 4 & 4 & 28 & 2 & 3 & 71 & 0 & 2 & 21 \\
YFJS05 & 8 & 7 & 4 & 4 & 32 & 2 & 3 & 81 & 0 & 2 & 24 \\
YFJS06 & 9 & 7 & 4 & 4 & 36 & 2 & 3 & 95 & 0 & 2 & 27 \\
YFJS07 & 9 & 7 & 4 & 4 & 36 & 2 & 3 & 93 & 0 & 2 & 27 \\
YFJS08 & 9 & 12 & 4 & 4 & 36 & 2 & 3 & 100 & 0 & 2 & 27 \\
YFJS09 & 9 & 12 & 4 & 4 & 36 & 4 & 8 & 219 & 0 & 2 & 27 \\
YFJS10 & 10 & 12 & 4 & 4 & 40 & 1 & 3 & 113 & 0 & 2 & 30 \\
YFJS11 & 10 & 10 & 5 & 5 & 50 & 1 & 3 & 134 & 0 & 2 & 40 \\
YFJS12 & 10 & 10 & 5 & 5 & 50 & 2 & 3 & 133 & 0 & 2 & 40 \\
YFJS13 & 10 & 10 & 5 & 5 & 50 & 1 & 3 & 137 & 0 & 2 & 40 \\
YFJS14 & 13 & 26 & 17 & 17 & 221 & 2 & 3 & 641 & 0 & 2 & 208 \\
YFJS15 & 13 & 26 & 17 & 17 & 221 & 2 & 3 & 648 & 0 & 2 & 208 \\
YFJS16 & 13 & 26 & 17 & 17 & 221 & 2 & 3 & 633 & 0 & 2 & 208 \\
YFJS17 & 17 & 26 & 17 & 17 & 289 & 3 & 5 & 1328 & 0 & 2 & 272 \\
YFJS18 & 17 & 26 & 17 & 17 & 289 & 3 & 5 & 1362 & 0 & 2 & 272 \\
YFJS19 & 17 & 26 & 17 & 17 & 289 & 3 & 5 & 1347 & 0 & 2 & 272 \\
YFJS20 & 17 & 26 & 17 & 17 & 289 & 3 & 5 & 1343 & 0 & 2 & 272 \\
\hline
\end{tabular}

Tabela 2.1: Descrição das instâncias com Y-jobs.

O primeiro conjunto de testes objetivou avaliar o desempenho do algoritmo list scheduling proposto. As Tabelas 2.5 e 2.6 mostram os resultado de aplicar o algoritmo list scheduling aos conjuntos de instâncias YFJS01-YFJS20 e DAFJS01-DAFJS30, respectivamente. Para cada instância, as tabelas mostram o makespan da solução obtida pelo algoritmo. Uma comparação com o makespan obtida pela heurística EST apresentada em Birgin et al. (2014) e o makespan obtido pelo solver com o limite de tempo de CPU de 1 hora e 10 horas também são apresentados. Em cada caso, se $v_{1}$ é o valor do makespan encontrado pelo list scheduling e $v_{2}$ é o valor encontrado pelo outro método, a diferença relativa "diff" é dada por diff $=\left(v_{1}-v_{2}\right) / v_{2}$. Isto significa que valores negativos de "diff" indicam que o algoritmo list scheduling encontrou uma solução de melhor qualidade. Ambas as tabelas mostram que o list scheduling proposto encontra soluções melhores que a heurística construtiva determinística EST apresentada em Birgin et al. (2014). Quando o list scheduling é comparado contra o solver exato, os dois conjuntos de instâncias devem ser considerados separadamente. No conjunto de instâncias YFJS01-YFJS20, o qual o solver encontrou soluções ótimas na maioria dos casos, a diferença relativa é de aproximadamente 35\%. No conjunto de instâncias DAFJS01-DAFJS30, o qual o solver não foi capaz de encontrar soluções ótimas na maioria dos casos, a distância é de aproximadamente $7 \%$. Contudo, é importante notar que o algoritmo list scheduling proposto é executado em menos de uma fração de segundo para cada instância. Em todo caso, não objetiva-se comparar a heurística proposta com o solver simplesmente avaliando a qualidade das soluções obtidas.

O segundo conjunto de experimentos objetivou avaliar o desempenho do método Beam Search proposto. Similarmente a todo método heurístico, também é relevante avaliar a influência dos parâmetros do método em relação ao seu desempenho. Com este propósito, todas as 80 combinações de $\alpha \in\{0.25,0.5,0.75,1\}, \beta \in\{0.25,0.5,0.75,1\}$ e $\xi \in\{0,0.25,0.5,0.75,1\}$ foram consideradas. Para cada combinação de parâmetros, o método foi executado sobre os dois conjuntos de instâncias e calculou-se a média da diferença relativa à solução obtida pelo solver executado com limite de tempo de CPU definido em 1 hora e 10 horas. As Tabelas 2.7 e 2.8 mostram estas médias das distâncias relativas para os conjuntos de instâncias YFJS01-YFJS20 e DAFJS01-DAFJS30 respec- 


\begin{tabular}{|c|cc|ccc|ccc|ccc|}
\hline \multirow{2}{*}{ Nome } & \multirow{2}{*}{$n$} & $m$ & \multicolumn{2}{|c|}{ \# de operações } & \multicolumn{3}{|c|}{$\left|F_{i}\right|$} & \multicolumn{3}{|c|}{ de precedências } \\
\cline { 4 - 10 } & & & $\min$ & $\max$ & $\sum$ & $\min$ & $\max$ & $\sum$ & $\min$ & $\max$ & $\sum$ \\
\hline \hline DAFJS01 & 4 & 5 & 5 & 9 & 26 & 2 & 4 & 82 & 0 & 3 & 26 \\
DAFJS02 & 4 & 5 & 5 & 7 & 25 & 2 & 4 & 79 & 0 & 3 & 23 \\
DAFJS03 & 4 & 10 & 10 & 17 & 55 & 3 & 7 & 279 & 0 & 2 & 52 \\
DAFJS04 & 4 & 10 & 9 & 14 & 43 & 3 & 7 & 220 & 0 & 2 & 40 \\
DAFJS05 & 6 & 5 & 5 & 13 & 39 & 2 & 4 & 104 & 0 & 3 & 34 \\
DAFJS06 & 6 & 5 & 5 & 13 & 44 & 2 & 4 & 136 & 0 & 3 & 41 \\
DAFJS07 & 6 & 10 & 7 & 23 & 85 & 3 & 7 & 431 & 0 & 3 & 82 \\
DAFJS08 & 6 & 10 & 6 & 23 & 85 & 3 & 7 & 403 & 0 & 3 & 82 \\
DAFJS09 & 8 & 5 & 4 & 9 & 45 & 2 & 4 & 135 & 0 & 3 & 42 \\
DAFJS10 & 8 & 5 & 4 & 11 & 58 & 2 & 4 & 168 & 0 & 3 & 52 \\
DAFJS11 & 8 & 10 & 10 & 23 & 113 & 3 & 7 & 534 & 0 & 3 & 108 \\
DAFJS12 & 8 & 10 & 9 & 22 & 117 & 3 & 7 & 603 & 0 & 3 & 114 \\
DAFJS13 & 10 & 5 & 5 & 11 & 62 & 2 & 4 & 193 & 0 & 3 & 55 \\
DAFJS14 & 10 & 5 & 4 & 10 & 69 & 2 & 4 & 206 & 0 & 3 & 62 \\
DAFJS15 & 10 & 10 & 8 & 19 & 120 & 3 & 7 & 595 & 0 & 3 & 117 \\
DAFJS16 & 10 & 10 & 6 & 20 & 120 & 3 & 7 & 602 & 0 & 3 & 114 \\
DAFJS17 & 12 & 5 & 4 & 11 & 82 & 2 & 4 & 246 & 0 & 3 & 77 \\
DAFJS18 & 12 & 5 & 5 & 9 & 74 & 2 & 4 & 231 & 0 & 2 & 64 \\
DAFJS19 & 8 & 7 & 7 & 13 & 70 & 3 & 5 & 283 & 0 & 3 & 66 \\
DAFJS20 & 10 & 7 & 6 & 17 & 92 & 3 & 5 & 361 & 0 & 3 & 87 \\
DAFJS21 & 12 & 7 & 5 & 16 & 107 & 3 & 5 & 425 & 0 & 3 & 102 \\
DAFJS22 & 12 & 7 & 5 & 17 & 116 & 3 & 5 & 450 & 0 & 3 & 109 \\
DAFJS23 & 8 & 9 & 6 & 17 & 76 & 4 & 6 & 367 & 0 & 3 & 71 \\
DAFJS24 & 8 & 9 & 6 & 25 & 92 & 4 & 6 & 463 & 0 & 2 & 87 \\
DAFJS25 & 10 & 9 & 9 & 19 & 123 & 4 & 6 & 619 & 0 & 3 & 119 \\
DAFJS26 & 10 & 9 & 8 & 17 & 119 & 4 & 6 & 606 & 0 & 3 & 116 \\
DAFJS27 & 12 & 9 & 7 & 22 & 127 & 4 & 6 & 625 & 0 & 3 & 118 \\
DAFJS28 & 8 & 10 & 8 & 15 & 91 & 3 & 7 & 457 & 0 & 3 & 89 \\
DAFJS29 & 8 & 10 & 7 & 19 & 95 & 3 & 7 & 468 & 0 & 3 & 94 \\
DAFJS30 & 10 & 10 & 8 & 19 & 98 & 3 & 7 & 509 & 0 & 3 & 94 \\
\hline
\end{tabular}

Tabela 2.2: Descrição das instâncias nas quais as relações de precedências entre as operações são dadas por um DAG qualquer.

tivamente. Esta mesma informação também é apresentada graficamente nas Figuras 2.11a e 2.11b respectivamente. Além disso, as Figuras 2.12a e 2.12b apresentam a média do tempo de CPU, em segundos, que o Beam Search utilizou para cada combinação de parâmetros.

Com foco na comparação do método Beam Search com as soluções obtidas pelo solver exato com limite de tempo de CPU de 1 hora (a outra comparação é similar), a Tabela 2.7 mostra que o método Beam Search com a combinação $(\alpha, \beta, \xi)=(1,1,1)$ obtém média das diferenças relativas de $3.5 \%$ no conjunto de instâncias YFJS01-YFJS20. Este é um resultado interessante se recordarmos que, para este conjunto de instâncias, o solver exato encontrou soluções ótimas na maioria dos casos. No outro extremo da tabela, com parâmetros $(\alpha, \beta, \xi)=(0.25,0.25,0)$, a média das diferenças relativas é de $8.86 \%$. Por outro lado, a Figura 2.12a mostra que a primeira combinação de parâmetros citada utiliza quase 2650 segundos por instância em média enquanto a última combinação de parâmetros utiliza aproximadamente 5 segundos por instância em média. Além disso, a tabela mostra que, considerando todas as 80 combinações de parâmetros, a diferença de $3.5 \%$ para $9.03 \%$ aponta uma importante característica: o desempenho do método não é fortemente dependente de uma escolha precisa de parâmetros. A relação entre a escolha de parâmetros e o desempenho do método pode 


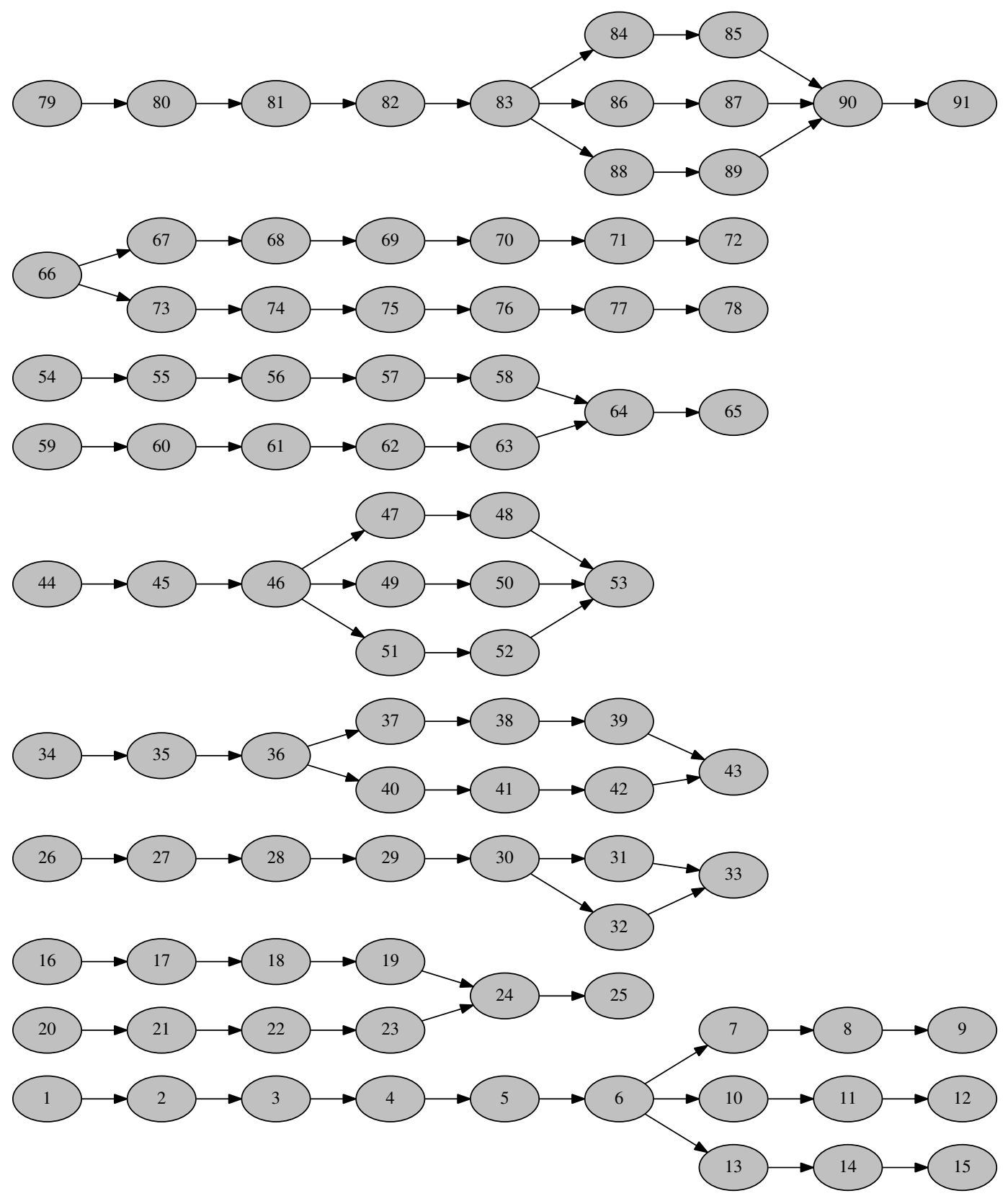

Figura 2.10: Representação gráfica das precedências entre as operações da instância DAFJS28.

ser facilmente vista nas Figuras 2.11a e 2.11b. Para valores fixos de $\alpha$ e $\beta$, o melhor valor para o parâmetro $\xi$ é sempre maior ou igual a 0.5. Além disso, como esperado, quanto maior os valores de $\alpha$ e $\beta$, maior o espaço de busca e em consequência, o método obtém soluções de melhor qualidade em troca de mais tempo de CPU.

Analisando a Tabela 2.8 é possível notar que, no conjunto de instâncias DAFJS01-DAFJS30, o qual o solver exato não foi capaz de encontrar soluções ótimas na maioria dos casos, o método Beam Search supera em qualidade as soluções do solver apresentando diferenças que vão de $-4.94 \%$, para a combinação de parâmetros $(\alpha, \beta, \xi)=(0.5,0.25,0)$, até $-6.36 \%$, para a combinação de parâmetros $(\alpha, \beta, \xi)=(1,1,0.5)$. No primeiro caso, considerando as instâncias DAFJS01-DAFJS30 individualmente, a diferença vai de $12.94 \%$ até $-17.18 \%$ com média de tempo de CPU de 1.18 segundos. No segundo caso, a diferença vai de $8.95 \%$ até $-17.52 \%$ com média de tempo de CPU de 115.04 segundos por instância. É importante lembrar que esta comparação é realizada em relação ao solver exato que utilizou um limite de tempo de CPU máximo de 1 hora e que este limite foi 


\begin{tabular}{|c|c|c|c|c|}
\hline \multirow{2}{*}{ Instância } & \multicolumn{2}{|c|}{ CPLEX com 1h de limite de tempo } & \multicolumn{2}{c|}{ CPLEX com 10h de limite de tempo } \\
\cline { 2 - 5 } & $\mathrm{mks}$ & CPU(s) & mks & CPU(s) \\
\hline \hline YFJS01 & 773 & 10.83 & - & - \\
YFJS02 & 825 & 9.25 & - & - \\
YFJS03 & 347 & 3.50 & - & - \\
YFJS04 & 390 & 3.31 .38 & - & - \\
YFJS05 & 445 & 3600 & - & 15072.02 \\
YFJS06 & {$[427 ; 447] 4.47 \%$} & 1277.35 & 446 & - \\
YFJS07 & 444 & 0.59 & - & - \\
YFJS08 & 353 & 13.09 & - & - \\
YFJS09 & 242 & 3.83 & - & - \\
YFJS10 & 399 & 168.72 & - & - \\
YFJS11 & 526 & 2843.79 & - & - \\
YFJS12 & 512 & 1427.47 & - & - \\
YFJS13 & 405 & 3378.27 & - & 36000 \\
YFJS14 & 1317 & 3600 & {$[1239 ; 1244] 0.40 \%$} & 36000 \\
YFJS15 & {$[1239 ; 1244] 0.40 \%$} & 3600 & {$[1222 ; 1231] 0.73 \%$} & 36000 \\
YFJS16 & {$[1222 ; 1243] 1.69 \%$} & 3600 & {$[1133 ; 1290] 12.17 \%$} & 3600 \\
YFJS17 & {$[1133 ; 1622] 30.15 \%$} & 3600 & {$[1220 ; 1499] 18.61 \%$} & 3600 \\
YFJS18 & {$[1220 ; 2082] 41.40 \%$} & 3600 & 36000 \\
YFJS19 & {$[926 ; 1525] 39.28 \%$} & 3600 & {$[926 ; 1333] 30.53 \%$} & 3600 \\
YFJS20 & {$[968 ; 2020] 52.08 \%$} & 3600 & {$[968 ; 1279] 24.32 \%$} & $36 \%$ \\
\hline
\end{tabular}

Tabela 2.3: Resultados numéricos da aplicação do CPLEX ao modelo (2.1-2.8) com as instâncias YFJS01-YFJS20. Como em Birgin et al. (2014), uma solução viável inicial é computada com a heurística EST (Birgin et al., 2014) e passada ao CPLEX.

atingido na maioria dos casos, como mostrado na Tabela 2.4. Portanto, isto significa que o método Beam Search foi capaz de encontrar melhores soluções utilizando menos tempo de CPU.

\subsection{Experimentos com FJSP clássico}

Uma vez que o FJSP clássico é um caso particular do FJSP-SF abordado neste trabalho, alguns experimentos com o método Beam Search deste trabalho utilizando instâncias clássicas do FJSP foram realizados. Para estes experimentos, foram considerados os conjuntos de instâncias introduzidos em Brandimarte (1993), em Dauzère-Pérès e Paulli (1997), em Barnes e Chambers (1996) e em Hurink et al. (1994). A Tabela 2.9 apresenta o erro relativo (RE) em relação aos limitantes inferiores disponíveis em http://people.idsia.ch/ monaldo/fjspresults/fjsp_result.ps. O RE é dado por

$$
R E=100 \% \times\left(m k s-m k s_{L B}\right) / m k s_{L B}
$$

onde $m k s$ é o makespan obtido pelo método Beam Search e $m k s_{L B}$ é o limitante inferior. Na tabela, "\# inst." é a quantidade de instâncias em cada conjunto.

Para meios de comparação, a Tabela 2.10 apresenta os resultados do algoritmo genético considerado estado da arte de Pezzella et al. (2008) para o FJSP. É possível ver que, mesmo quando aplicado à instâncias do FJSP clássico, o método Beam Search obteve resultados competitivos. De acordo com as tabelas, o Beam Search supera todos os três GA's nos conjuntos de instâncias Dauzére-Péérés and Paulli e Hurink VData. É importante dizer que, estes dois conjuntos onde o Beam Search supera os resultados dos GA's, possuem a maior flexibilidade nas máquinas, quer dizer, são os conjuntos dos quais cada operação possui a maior quantidade de possíveis máquinas de processamento.

É importante apontar alguns aspectos para a comparação dos resultados apresentados nesta 


\begin{tabular}{|c|c|c|c|c|}
\hline \multirow{2}{*}{ Instância } & \multicolumn{2}{|c|}{ CPLEX com $1 \mathrm{~h}$ de limite de tempo } & \multicolumn{2}{|c|}{ CPLEX com $10 \mathrm{~h}$ de limite de tempo } \\
\hline & mks & $\mathrm{CPU}(\mathrm{s})$ & mks & $\mathrm{CPU}(\mathrm{s})$ \\
\hline DAFJS01 & 257 & 50.17 & - & - \\
\hline DAFJS02 & 289 & 794.66 & - & - \\
\hline DAFJS03 & 576 & 10.22 & - & - \\
\hline DAFJS04 & 606 & 0.76 & - & - \\
\hline DAFJS05 & {$[351.57 ; 402] 12.54 \%$} & 3600 & {$[381 ; 394] 3.30 \%$} & 36000 \\
\hline DAFJS06 & {$[326 ; 431] 24.36 \%$} & 3600 & {$[326 ; 410] 20.49 \%$} & 36000 \\
\hline DAFJS07 & {$[497.90 ; 565] 11.88 \%$} & 3600 & {$[498.22 ; 547] 8.92 \%$} & 36000 \\
\hline DAFJS08 & {$[628 ; 631] 0.48 \%$} & 3600 & {$[628 ; 631] 0.48 \%$} & 36000 \\
\hline DAFJS09 & {$[315 ; 484] 34.92 \%$} & 3600 & {$[316.74 ; 471] 32.75 \%$} & 36000 \\
\hline DAFJS10 & {$[336 ; 569] 40.95 \%$} & 3600 & {$[336 ; 538] 37.55 \%$} & 36000 \\
\hline DAFJS11 & {$[658 ; 708] 7.06 \%$} & 3600 & {$[658 ; 701] 6.13 \%$} & 36000 \\
\hline DAFJS12 & {$[530 ; 720] 26.39 \%$} & 3600 & {$[530 ; 720] 26.39 \%$} & 36000 \\
\hline DAFJS13 & {$[304 ; 710] 57.18 \%$} & 3600 & {$[304 ; 683] 55.49 \%$} & 36000 \\
\hline DAFJS14 & {$[358.95 ; 838] 57.17 \%$} & 3600 & {$[358.95 ; 775] 53.68 \%$} & 36000 \\
\hline DAFJS15 & {$[512 ; 818] 37.41 \%$} & 3600 & {$[512 ; 796] 35.68 \%$} & 36000 \\
\hline DAFJS16 & {$[640 ; 831] 22.98 \%$} & 3600 & {$[640 ; 798] 19.80 \%$} & 36000 \\
\hline DAFJS17 & {$[300 ; 904] 66.81 \%$} & 3600 & {$[300 ; 902] 66.74 \%$} & 36000 \\
\hline DAFJS18 & {$[322 ; 951] 66.14 \%$} & 3600 & {$[322 ; 878] 63.33 \%$} & 36000 \\
\hline DAFJS19 & {$[512 ; 595] 13.95 \%$} & 3600 & {$[512 ; 585] 12.48 \%$} & 36000 \\
\hline DAFJS20 & {$[434 ; 815] 46.75 \%$} & 3600 & {$[434 ; 810] 46.42 \%$} & 36000 \\
\hline DAFJS21 & {$[504 ; 965] 47.77 \%$} & 3600 & {$[504 ; 959] 47.45 \%$} & 36000 \\
\hline DAFJS22 & {$[464 ; 902] 48.56 \%$} & 3600 & {$[464 ; 896] 48.21 \%$} & 36000 \\
\hline DAFJS23 & [450;541] $16.82 \%$ & 3600 & {$[450 ; 537] 16.20 \%$} & 36000 \\
\hline DAFJS24 & {$[476 ; 660] 27.88 \%$} & 3600 & {$[476 ; 648] 26.54 \%$} & 36000 \\
\hline DAFJS25 & {$[584 ; 897] 34.89 \%$} & 3600 & {$[584 ; 879] 33.56 \%$} & 36000 \\
\hline DAFJS26 & {$[565 ; 903] 37.43 \%$} & 3600 & {$[565 ; 898] 37.08 \%$} & 36000 \\
\hline DAFJS27 & {$[503 ; 981] 48.73 \%$} & 3600 & {$[503 ; 981] 48.73 \%$} & 36000 \\
\hline DAFJS28 & {$[535 ; 662] 19.18 \%$} & 3600 & {$[535 ; 572] 6.47 \%$} & 36000 \\
\hline DAFJS29 & {$[609 ; 720] 15.42 \%$} & 3600 & {$[609 ; 710] 14.23 \%$} & 36000 \\
\hline DAFJS30 & {$[467 ; 637] 26.69 \%$} & 3600 & {$[467 ; 615] 24.07 \%$} & 36000 \\
\hline
\end{tabular}

Tabela 2.4: Resultados numéricos da aplicação do CPLEX ao modelo (2.1-2.8) com as instâncias DAFJS01-DAFJS30. Como em Birgin et al. (2014), uma solução factivel inicial é computada com a heurística EST Birgin et al. (2014) e passada ao CPLEX.

seção. Primeiramente, o método Beam Search é um método determinístico, sem aleatoriedade e com tempo finito para conclusão. Os GA's apresentados foram executados cinco vezes e apenas o melhor resultado foi apresentado. Um outro aspecto importante é o fato de que o método Beam Search foi executado fixando os parâmetros $(\alpha, \beta, \xi)=(1,1,1)$ enquanto para os experimentos do FJSP-SF, 80 combinações diferentes foram avaliadas. Os métodos utilizados para comparação foram executados em diferentes máquinas e o tempo de CPU dos GA's de Pezzella et al. (2008) não foram reportados. Por último e não menos importante, vale a pena destacar que o método Beam Search deste trabalho foi desenvolvido para ser aplicado ao FJSP-SF, que possui como caso particular o FJSP clássico.

\subsection{Conclusão}

Este capítulo aborda uma extensão do FJSP na qual as precedências entre as operações de uma tarefa são definidas através de um DAG qualquer. Inicialmente, um algoritmo LS baseado em regras que exploram várias características do problema para determinar um par operação/máquina para 


\begin{tabular}{|c|c|cc|cc|cc|}
\hline \multirow{2}{*}{ Instância } & \multirow{2}{*}{ mks } & \multicolumn{2}{|c|}{ heuristica EST } & \multicolumn{2}{c|}{ CPLEX (limite 1h) } & \multicolumn{2}{c|}{ CPLEX (limite 10h) } \\
\cline { 3 - 8 } & & mks & diff & mks & diff & mks & diff \\
\hline \hline YFJS01 & 1130 & 1318 & -14.26 & 773 & 46.18 & 773 & 46.18 \\
YFJS02 & 1133 & 1243 & -8.85 & 825 & 37.33 & 825 & 37.33 \\
YFJS03 & 575 & 439 & 30.98 & 347 & 65.71 & 347 & 65.71 \\
YFJS04 & 576 & 569 & 1.23 & 390 & 47.69 & 390 & 47.69 \\
YFJS05 & 608 & 566 & 7.42 & 445 & 36.63 & 445 & 36.63 \\
YFJS06 & 633 & 633 & 0.00 & 447 & 41.61 & 446 & 41.93 \\
YFJS07 & 628 & 628 & 0.00 & 444 & 41.44 & 444 & 41.44 \\
YFJS08 & 485 & 531 & -8.66 & 353 & 37.39 & 353 & 37.39 \\
YFJS09 & 402 & 506 & -20.55 & 242 & 66.12 & 242 & 66.12 \\
YFJS10 & 513 & 541 & -5.18 & 399 & 28.57 & 399 & 28.57 \\
YFJS11 & 745 & 740 & 0.68 & 526 & 41.63 & 526 & 41.63 \\
YFJS12 & 744 & 813 & -8.49 & 512 & 45.31 & 512 & 45.31 \\
YFJS13 & 553 & 717 & -22.87 & 405 & 36.54 & 405 & 36.54 \\
YFJS14 & 1555 & 2055 & -24.33 & 1317 & 18.07 & 1317 & 18.07 \\
YFJS15 & 1690 & 2296 & -26.39 & 1244 & 35.85 & 1244 & 35.85 \\
YFJS16 & 1769 & 2006 & -11.81 & 1243 & 42.32 & 1231 & 43.70 \\
YFJS17 & 1734 & 2408 & -27.99 & 1622 & 6.91 & 1290 & 34.42 \\
YFJS18 & 1735 & 2082 & -16.67 & 2082 & -16.67 & 1499 & 15.74 \\
YFJS19 & 1604 & 2038 & -21.30 & 1525 & 5.18 & 1333 & 20.33 \\
YFJS20 & 1700 & 2369 & -28.24 & 2020 & -15.84 & 1279 & 32.92 \\
\hline \hline \multicolumn{2}{|c|}{ Média } & \multicolumn{7}{|c|}{-10.26} & 32.40 & & 38.68 \\
\hline
\end{tabular}

Tabela 2.5: Resultados do algoritmo list scheduling aplicado ao conjunto de instâncias YFJS01-YFJS20.

ser programado a cada iteração foi cuidadosamente desenvolvido. A partir daí, uma extensão natural do LS que explora um conjunto de pares operação/máquina, ao invés de apenas um par, originou o BS deste trabalho. Para avaliar o desempenho dos métodos propostos, uma solução inicial foi gerada a partir de uma heurística construtiva proposta em Birgin et al. (2014) e então um solver exato foi aplicado ao modelo (2.1-2.8) introduzido também em Birgin et al. (2014). O solver foi executado com um limite de 1 e de 10 horas. Um total de 50 instâncias para o FJSP-SF foram utilizadas nos experimentos numéricos. Os resultados obtidos demostram a eficiência das heurísticas propostas que, em linhas gerais, conseguem obter soluções melhores utilizando menos tempo de CPU quando comparados ao único método encontrado na literatura para o problema.

Além disso, 6 conjuntos clássicos de instâncias do FJSP também foram avaliados. Os resultados foram comparados com os algoritmos genéticos (GA) considerados estado da arte em Pezzella et al. (2008). Os GAs foram executados 5 vezes e somente o melhor resultado foi considerado. Em linhas gerais, o método BS proposto nesta tese se mostrou competitivo e até mesmo superou todos os GAs nos conjuntos mais complexos.

Todos os métodos e instâncias deste capítulo estão disponíveis em http://www.ime.usp.br/ egbirgin/. 


\begin{tabular}{|c|c|cc|cc|cc|}
\hline \multirow{2}{*}{ Instância } & \multirow{2}{*}{ mks } & \multicolumn{2}{|c|}{ heuristica EST } & \multicolumn{2}{|c|}{ CPLEX (limite 1h) } & \multicolumn{2}{l|}{ CPLEX (limite 10h) } \\
\cline { 3 - 8 } & & mks & diff & mks & diff & mks & diff \\
\hline \hline DAFJS01 & 321 & 327 & -1.83 & 257 & 24.90 & 257 & 24.90 \\
DAFJS02 & 350 & 382 & -8.38 & 289 & 21.11 & 289 & 21.11 \\
DAFJS03 & 631 & 710 & -11.13 & 576 & 9.55 & 576 & 9.55 \\
DAFJS04 & 607 & 653 & -7.04 & 606 & 0.17 & 606 & 0.17 \\
DAFJS05 & 505 & 482 & 4.77 & 402 & 25.62 & 394 & 28.17 \\
DAFJS06 & 497 & 489 & 1.64 & 431 & 15.31 & 410 & 21.22 \\
DAFJS07 & 632 & 717 & -11.85 & 565 & 11.86 & 547 & 15.54 \\
DAFJS08 & 706 & 847 & -16.65 & 631 & 11.89 & 631 & 11.89 \\
DAFJS09 & 533 & 535 & -0.37 & 484 & 10.12 & 471 & 13.16 \\
DAFJS10 & 621 & 629 & -1.27 & 569 & 9.14 & 538 & 15.43 \\
DAFJS11 & 767 & 708 & 8.33 & 708 & 8.33 & 701 & 9.42 \\
DAFJS12 & 727 & 720 & 0.97 & 720 & 0.97 & 720 & 0.97 \\
DAFJS13 & 768 & 766 & 0.26 & 710 & 8.17 & 683 & 12.45 \\
DAFJS14 & 888 & 871 & 1.95 & 838 & 5.97 & 775 & 14.58 \\
DAFJS15 & 788 & 818 & -3.67 & 818 & -3.67 & 796 & -1.01 \\
DAFJS16 & 808 & 831 & -2.77 & 831 & -2.77 & 798 & 1.25 \\
DAFJS17 & 935 & 910 & 2.75 & 904 & 3.43 & 902 & 3.66 \\
DAFJS18 & 939 & 951 & -1.26 & 951 & -1.26 & 878 & 6.95 \\
DAFJS19 & 598 & 601 & -0.50 & 595 & 0.50 & 585 & 2.22 \\
DAFJS20 & 854 & 815 & 4.79 & 815 & 4.79 & 810 & 5.43 \\
DAFJS21 & 937 & 965 & -2.90 & 965 & -2.90 & 959 & -2.29 \\
DAFJS22 & 826 & 902 & -8.43 & 902 & -8.43 & 896 & -7.81 \\
DAFJS23 & 548 & 632 & -13.29 & 541 & 1.29 & 537 & 2.05 \\
DAFJS24 & 687 & 674 & 1.93 & 660 & 4.09 & 648 & 6.02 \\
DAFJS25 & 885 & 897 & -1.34 & 897 & -1.34 & 879 & 0.68 \\
DAFJS26 & 915 & 903 & 1.33 & 903 & 1.33 & 898 & 1.89 \\
DAFJS27 & 982 & 981 & 0.10 & 981 & 0.10 & 981 & 0.10 \\
DAFJS28 & 633 & 703 & -9.96 & 662 & -4.38 & 572 & 10.66 \\
DAFJS29 & 800 & 760 & 5.26 & 720 & 11.11 & 710 & 12.68 \\
DAFJS30 & 640 & 657 & -2.59 & 637 & 0.47 & 615 & 4.07 \\
\hline \hline Média & & & -2.20 & & 5.73 & & 8.40 \\
\hline
\end{tabular}

Tabela 2.6: Resultados do algoritmo list scheduling aplicado ao conjunto de instâncias DAFJS01-DAFJS30. 


\begin{tabular}{|c|c|c|c|c|c|c|c|c|c|}
\hline \multirow{3}{*}{$\alpha$} & \multirow{3}{*}{$\xi$} & \multirow{2}{*}{\multicolumn{4}{|c|}{$\begin{array}{c}\text { diff vs. CPLEX (limite 1h) } \\
\beta\end{array}$}} & \multirow{2}{*}{\multicolumn{4}{|c|}{$\begin{array}{l}\text { diff vs. CPLEX (limite 10h) } \\
\beta\end{array}$}} \\
\hline & & & & & & & & & \\
\hline & & 0.25 & 0.50 & 0.75 & 1.00 & 0.25 & 0.50 & 0.75 & 1.00 \\
\hline \multirow{5}{*}{0.25} & 0.00 & 8.86 & $\overline{8.87}$ & $\overline{8.42}$ & 8.28 & $\overline{13.94}$ & 13.91 & 13.47 & 13.32 \\
\hline & 0.25 & 7.73 & 7.17 & 6.51 & 6.25 & 12.72 & 12.15 & 11.45 & 11.18 \\
\hline & 0.50 & 7.17 & 6.42 & 5.70 & 5.68 & 12.09 & 11.32 & 10.59 & 10.56 \\
\hline & 0.75 & 7.76 & 7.02 & 6.54 & 6.22 & 12.74 & 11.98 & 11.49 & 11.17 \\
\hline & 1.00 & 8.32 & 7.68 & 6.80 & 6.46 & 13.31 & 12.65 & 11.75 & 11.41 \\
\hline \multirow{5}{*}{0.50} & 0.00 & 8.76 & 7.45 & 7.41 & 7.24 & 13.80 & 12.46 & 12.41 & 12.24 \\
\hline & 0.25 & 7.14 & 6.00 & 5.22 & 5.05 & 12.05 & 10.88 & 10.07 & 9.90 \\
\hline & 0.50 & 6.97 & 5.80 & 5.69 & 5.23 & 11.93 & 10.74 & 10.62 & 10.17 \\
\hline & 0.75 & 6.82 & 6.11 & 5.65 & 5.21 & 11.72 & 11.00 & 10.54 & 10.09 \\
\hline & 1.00 & 6.51 & 5.80 & 5.46 & 5.50 & 11.46 & 10.75 & 10.40 & 10.43 \\
\hline \multirow{5}{*}{0.75} & 0.00 & 9.03 & 6.86 & 6.87 & 6.51 & 14.04 & 11.87 & 11.88 & 11.54 \\
\hline & 0.25 & 6.44 & 4.80 & 4.24 & 4.19 & 11.35 & 9.69 & 9.09 & 9.04 \\
\hline & 0.50 & 6.01 & 5.11 & 4.80 & 4.48 & 10.91 & 9.99 & 9.68 & 9.35 \\
\hline & 0.75 & 5.50 & 4.53 & 4.32 & 3.81 & 10.44 & 9.45 & 9.24 & 8.73 \\
\hline & 1.00 & 4.99 & 4.08 & 4.00 & 3.82 & 9.93 & 8.99 & 8.91 & 8.73 \\
\hline \multirow{5}{*}{1.00} & 0.00 & 9.03 & 6.85 & 7.11 & 6.86 & 14.05 & 11.86 & 12.09 & 11.87 \\
\hline & 0.25 & 6.53 & 4.63 & 4.59 & 4.42 & 11.43 & 9.48 & 9.44 & 9.27 \\
\hline & 0.50 & 5.41 & 4.66 & 4.43 & 4.00 & 10.30 & 9.54 & 9.31 & 8.86 \\
\hline & 0.75 & 5.12 & 4.32 & 4.01 & 3.87 & 9.99 & 9.19 & 8.84 & 8.71 \\
\hline & 1.00 & 4.56 & 3.79 & 3.92 & 3.50 & 9.49 & 8.70 & 8.80 & 8.38 \\
\hline
\end{tabular}

Tabela 2.7: Médias dos desvios relativos obtidos na comparação dos resultados do método Beam Search, com diferentes escolhas de parâmetros para $\alpha, \beta, e \xi$, em relação ao CPLEX solver com dois limites de tempo de CPU (1 hora e 10 horas), quando aplicados às instâncias YFJS01-YFJS20. 


\begin{tabular}{|c|c|c|c|c|c|c|c|c|c|}
\hline & \multirow[b]{3}{*}{ ( } & \multirow{2}{*}{\multicolumn{4}{|c|}{$\frac{\text { diff vs. CPLEX (limite 1h) }}{\beta}$}} & \multirow{2}{*}{\multicolumn{4}{|c|}{$\frac{\text { diff vs. CPLEX (limite 10h) }}{\beta}$}} \\
\hline \multirow{2}{*}{$\alpha$} & & & & & & & & & \\
\hline & & 0.25 & 0.50 & 0.75 & 1.00 & 0.25 & 0.50 & 0.75 & 1.00 \\
\hline \multirow{5}{*}{0.25} & 0.00 & -5.07 & -5.57 & -5.75 & -5.72 & -2.69 & -3.20 & -3.38 & -3.35 \\
\hline & 0.25 & -5.18 & -5.74 & -5.95 & -6.01 & -2.80 & -3.37 & -3.60 & -3.65 \\
\hline & 0.50 & -5.24 & -5.70 & -5.81 & -5.91 & -2.86 & -3.33 & -3.44 & -3.55 \\
\hline & 0.75 & -5.20 & -5.67 & -5.85 & -5.90 & -2.81 & -3.30 & -3.49 & -3.54 \\
\hline & 1.00 & -5.19 & -5.66 & -5.87 & -6.03 & -2.80 & -3.28 & -3.50 & -3.68 \\
\hline \multirow{5}{*}{0.50} & 0.00 & -4.94 & -5.56 & -5.75 & -5.77 & -2.55 & -3.19 & -3.39 & -3.41 \\
\hline & 0.25 & -5.53 & -6.01 & -6.14 & -6.19 & -3.16 & -3.65 & -3.79 & -3.84 \\
\hline & 0.50 & -5.71 & -6.07 & -6.13 & -6.19 & -3.36 & -3.72 & -3.78 & -3.85 \\
\hline & 0.75 & -5.43 & -5.91 & -6.06 & -6.11 & -3.05 & -3.54 & -3.70 & -3.75 \\
\hline & 1.00 & -5.11 & -5.86 & -5.98 & -5.96 & -2.72 & -3.49 & -3.62 & -3.60 \\
\hline \multirow{5}{*}{0.75} & 0.00 & -5.19 & -5.62 & -5.70 & -5.77 & -2.81 & -3.25 & -3.33 & -3.40 \\
\hline & 0.25 & -5.88 & -6.02 & -6.07 & -6.25 & -3.52 & -3.66 & -3.72 & -3.90 \\
\hline & 0.50 & -5.82 & -6.06 & -6.27 & -6.32 & -3.46 & -3.71 & -3.93 & -3.98 \\
\hline & 0.75 & -5.68 & -5.93 & -6.16 & -6.25 & -3.32 & -3.58 & -3.81 & -3.90 \\
\hline & 1.00 & -5.74 & -6.07 & -6.19 & -6.34 & -3.37 & -3.71 & -3.83 & -3.99 \\
\hline \multirow{5}{*}{1.00} & 0.00 & -5.19 & -5.65 & -5.70 & -5.77 & -2.81 & -3.27 & -3.33 & -3.40 \\
\hline & 0.25 & -5.90 & -6.10 & -6.30 & -6.33 & -3.53 & -3.74 & -3.95 & -3.98 \\
\hline & 0.50 & -6.01 & -6.28 & -6.36 & -6.36 & -3.65 & -3.94 & -4.02 & -4.02 \\
\hline & 0.75 & -5.72 & -6.04 & -6.19 & -6.29 & -3.36 & -3.69 & -3.84 & -3.95 \\
\hline & 1.00 & -5.65 & -6.01 & -6.13 & -6.22 & -3.28 & -3.65 & -3.77 & -3.86 \\
\hline
\end{tabular}

Tabela 2.8: Médias dos desvios relativos obtidos na comparação dos resultados do método Beam Search, com diferentes escolhas de parâmetros para $\alpha, \beta$, e $\xi$, em relação ao CPLEX solver com dois limites de tempo de CPU (1 hora e 10 horas), quando aplicados às instâncias DAFJS01-DAFJS30.

\begin{tabular}{|l|c|r|}
\hline Instâncias & \# inst. & RE \\
\hline \hline Brandimarte & 10 & 26.54 \\
Dauzére-Péérés and Paulli & 18 & 6.21 \\
Barnes and Chambers & 21 & 32.09 \\
Hurink EData & 43 & 8.52 \\
Hurink RData & 43 & 4.75 \\
Hurink VData & 43 & 0.26 \\
\hline
\end{tabular}

Tabela 2.9: Erros relativos obtidos pelo método Beam Search aplicado à vários conjuntos clássicos de instâncias do FJSP.

\begin{tabular}{|l|c|ccc|}
\hline Instâncias & \# inst. & Pezzella et al. (2008) & Chen et al. (1999) & Jia et al. (2003) \\
\hline \hline Brandimarte & 10 & 17.53 & 19.55 & 19.11 \\
Dauzére-Péérés and Paulli & 18 & 7.63 & 7.91 & 10.62 \\
Barnes and Chambers & 21 & 29.56 & 38.64 & 29.75 \\
Hurink EData & 43 & 6.00 & 5.59 & 9.01 \\
Hurink RData & 43 & 4.42 & 4.41 & 8.34 \\
Hurink VData & 43 & 2.04 & 2.59 & 3.24 \\
\hline
\end{tabular}

Tabela 2.10: Melhores erros relativos obtidos por algoritmos genéticos clássicos executados cinco vezes em cada conjunto de instância do FJSP. 


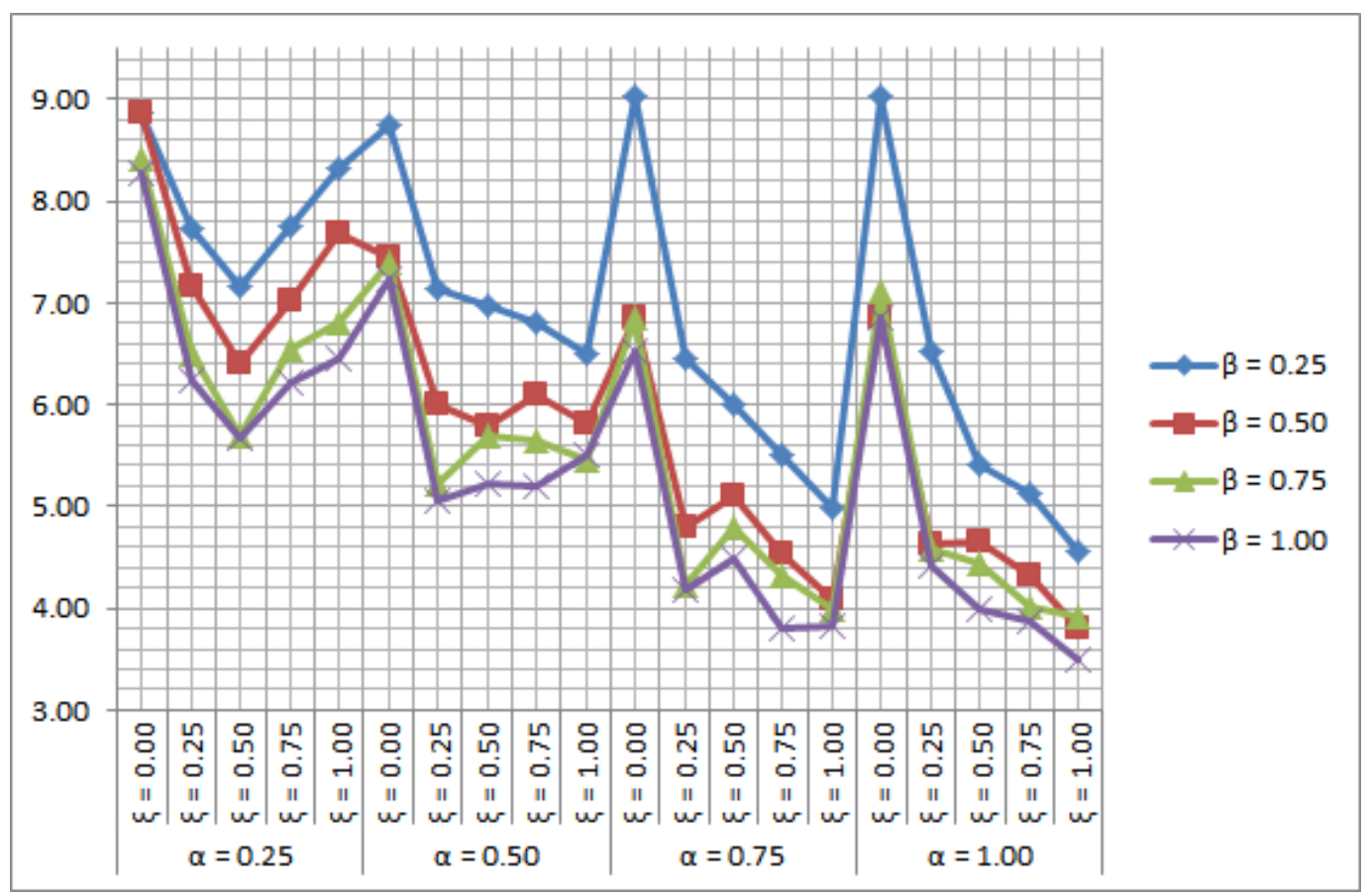

(a)

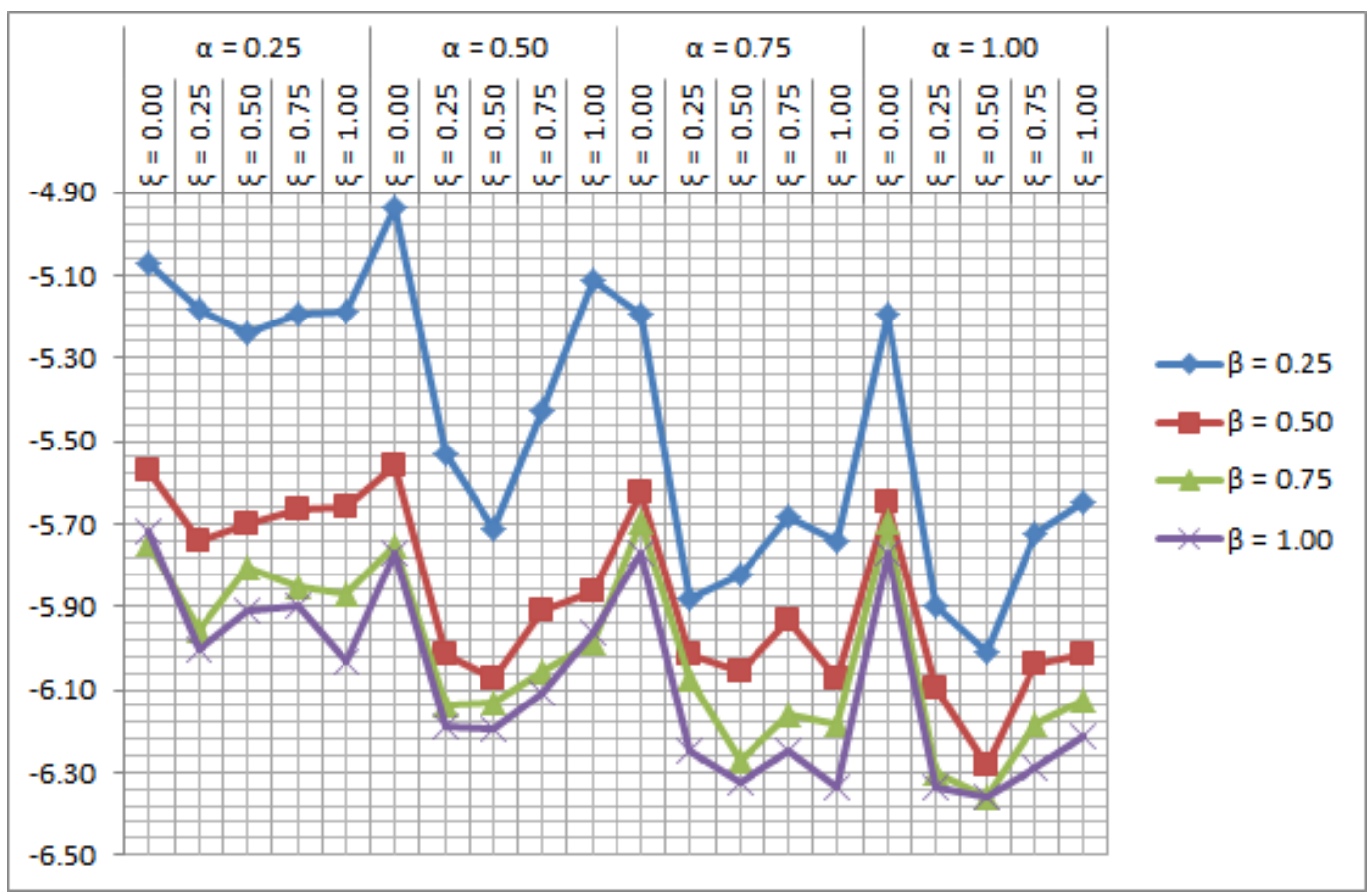

(b)

Figura 2.11: Representação gráfica do desempenho do método Beam Search, com diferentes escolhas de parâmetros $\alpha, \beta$ e $\xi$, quando comparados aos resultados obtidos pelo solver CPLEX executado com limite de tempo de 1 hora. (a) Instâncias YFJS01-YFJS20. (b) Instâncias DAFJS01-DAFJS30. 


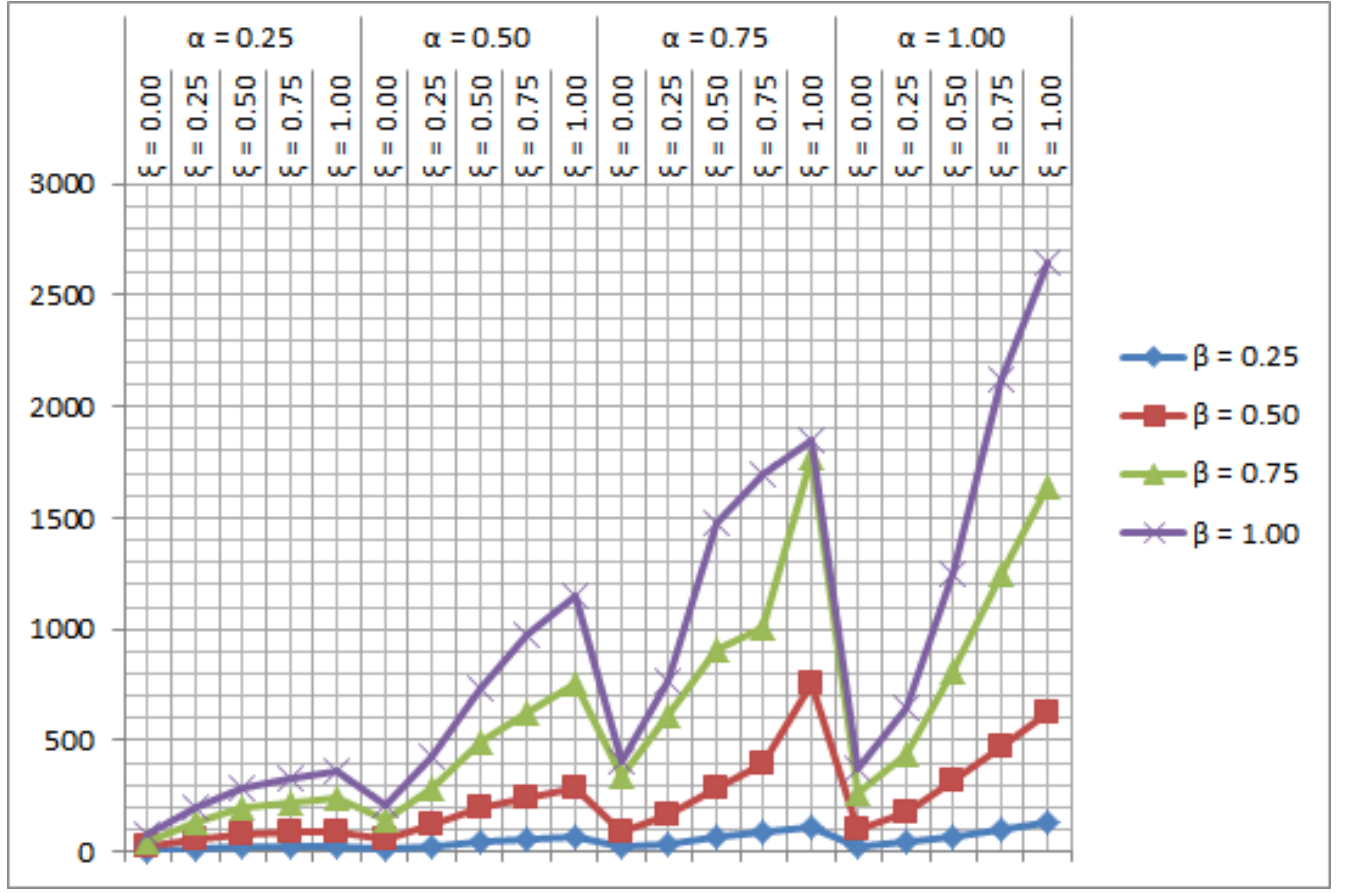

(a)

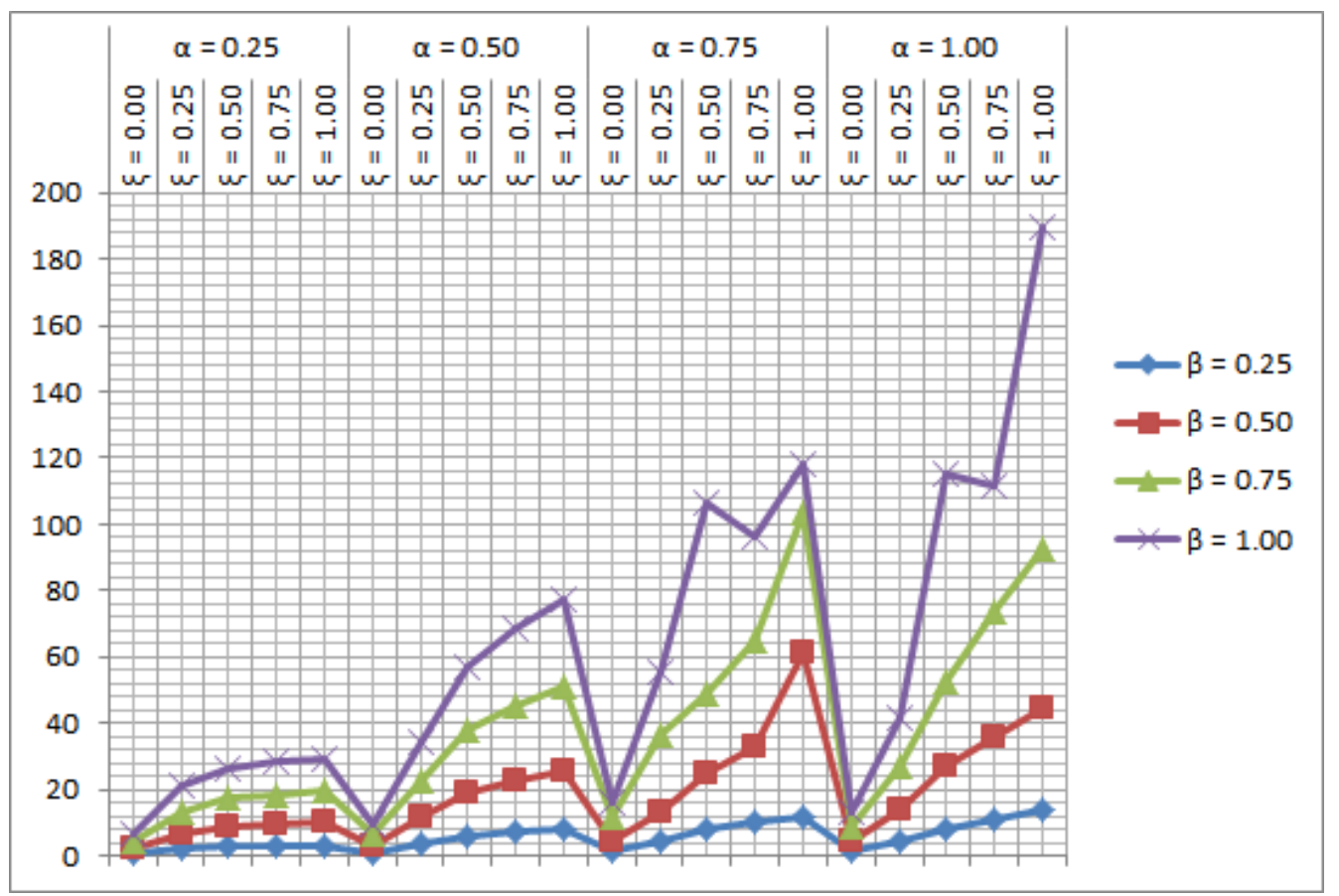

(b)

Figura 2.12: Representação gráfica do tempo de CPU em segundos requerido pelo método Beam Search, com diferentes escolhas de parâmetros $\alpha, \beta$ e $\xi$. (a) Instâncias YFJS01-YFJS20. (b) Instâncias DAFJS01DAFJS30. 


\section{Capítulo 3}

\section{Flowshop Scheduling Problem com Tempos de Espera}

O Flowshop Scheduling Problem (FSP) é um problema de produção onde $n$ tarefas devem ser processadas em $m$ máquinas. Cada tarefa é composta por $m$ operações e todas as tarefas possuem uma mesma rota de processamento através das máquinas, quer dizer, a $k$-ésima operação de uma tarefa $i$ qualquer sempre será processada pela $k$-ésima máquina do sistema com tempo de processamento $p_{i k}, k=1, \ldots, m$. Cada máquina pode processar as $n$ tarefas em uma mesma ordem ou não. Os cenários onde obrigatoriamente as $n$ tarefas devem ser processadas numa mesma ordem são chamados de permutation flowshops ou flowshops com permutação de sequência (Pinedo, 2008; Sakuraba et al., 2009). Em linhas gerais, o problema consiste em definir instantes de início de processamento $s_{i k}$ para cada tarefa $i$ na máquina $k$, respeitando a rota de processamento, de forma a não sobrepor nenhum dos processamentos nas máquinas e visando a minimização de algum objetivo.

É importante ressaltar que a rota de uma tarefa é o caminho que ela precisa percorrer entre as máquinas de processamento para que ela seja finalizada.

Pinedo (2008) aponta que o FSP pode possuir ou não capacidade ilimitada de armazenamento entre as máquinas. Esta característica é comum em indústrias que lidam com pequenos produtos, como a indústria de circuitos ou indústria de drogas farmacêuticas. Além disso, quando a capacidade entre as máquinas não é ilimitada, esse armazenamento pode ficar cheio e causar bloqueio no processamento das tarefas.

Alguns dos possíveis objetivos para o FSP consistem na minimização da somatória dos términos de processamento de todas as tarefas, na minimização do término de processamento da última tarefa do sistema (também conhecido como makespan), na minimização dos atrasos nas entregas das tarefas do sistema ou ainda na minimização da somatória dos adiantamentos e dos atrasos. Problemas que consideram adiantamentos ou atrasos consistem em problemas em que cada tarefa $i$ possui uma data de entrega $d_{i}, i=1, \ldots, n$, e assim, o adiantamento $E_{i}$ desta tarefa é definido como $\max \left\{0, d_{i}-C_{i m}\right\}$, onde $C_{i m}$ é o instante de término de processamento da tarefa $i$ na última máquina $m$. De maneira similar, o atraso $T_{i}$ é definido como $\max \left\{0, C_{i m}-d_{i}\right\}$. Pinedo (2008) menciona que problemas com datas de entrega são mais difíceis de se obter soluções do que os problemas que consideram makespan. Hall e Posner (1991) mostraram que o FSP com minimização da somatória dos adiantamentos e dos atrasos em uma única máquina é NP-Difícil, mesmo que todas as tarefas possuam a mesma data de entrega. O trabalho de Wan e Yuan (2013) prova que na verdade o problema é fortemente NP-Difícil.

O FSP com tempos de espera (FSP-WT) deste trabalho é uma variante do FSP clássico, descrito nos parágrafos anteriores, que considera: (a) a minimização dos adiantamentos e dos atrasos; (b) data de entrega única para todas as tarefas; (c) capacidade ilimitada de armazenamento entre as máquinas; (d) permutação de sequência; (e) proibição na interrupção do processamento de uma tarefa; (f) os tempos entre o término do processamento de uma tarefa em uma máquina até o início de processamento na próxima máquina. O objetivo é escolher dentre as soluções que minimizam os adiantamentos e os atrasos aquela que gere o menor tempo de espera das tarefas entre as máquinas. 
Fernandez-Viagas et al. (2016) classificam esse tipo de objetivo como um objetivo Just in Time.

Devido a complexidade do problema, somente alguns trabalhos com abordagens exatas (ou aproximadas) para o FSP com adiantamentos e atrasos podem ser encontrados na literatura. Nesta linha, Sarper (1995) propôs um MIP para o problema com duas máquinas e minimização do desvio médio absoluto das somatórias dos adiantamentos e atrasos. Nesse trabalho, Sarper (1995) só conseguiu encontrar resultados ótimos para instâncias com 5 e 6 tarefas. Moslehi et al. (2009) desenvolveram um algoritmo do tipo Branch and Bound para o FSP com duas máquinas e com minimização da somatória dos adiantamentos e atrasos. Moslehi et al. (2009) criaram um total de 380 instâncias, divididas em dois grupos de 220 e 160 respectivamente. No primeiro grupo, as instâncias possuíam data de entrega comum mais apertada enquanto no segundo grupo as instâncias possuíam data de entrega comum mais folgada. De acordo com os resultados obtidos por Moslehi et al. (2009), o algoritmo proposto conseguiu resolver problemas com até 1000 tarefas do primeiro grupo de instâncias mas somente com 20 tarefas no segundo grupo com tempo razoável. Chandra et al. (2009) fizeram uma análise sobre as diferentes datas de entrega para o FSP e afirmaram que datas de entrega muito apertadas transformam o problema de minimização dos adiantamentos e atrasos em um problema de minimização dos tempos de término de processamento das tarefas. Fernandez-Viagas et al. (2016) reforçaram essa afirmação e ainda mencionaram que datas de entrega muito apertadas geram funções objetivos opostas à datas de entrega folgadas.

Contudo, diversas abordagens heurísticas foram desenvolvidas para o FSP. Kanet (1981) desenvolveu uma heurística que encontra soluções ótimas para o FSP com uma única máquina e com adiantamentos e atrasos desde que a data de entrega seja maior que a somatória dos tempos de processamento das tarefas. Chandra et al. (2009) mostraram uma extensão natural deste resultado para o problema com $m$ máquinas. Sakuraba et al. (2009) combinaram uma heurística NEH (Nawaz et al., 1983) com uma busca tabu para construir um sequenciamento de tarefas no FSP com duas máquinas. Nesse trabalho, os instantes de início de processamento de cada operação era determinado por uma adaptação do algoritmo timing proposto em Hendel e Sourd (2007) para o problema com duas máquinas e data de entrega única. Sakuraba et al. (2009) analisaram ainda várias regras de despacho para servir como base para a heurística e concluíram que a regra do tipo shortest processing time (SPT) utilizando a somatória dos tempos de processamento obtinha melhores resultados quando comparada a outras 8 regras. Chandra et al. (2009) também propuseram uma busca tabu para o FSP com $m$ máquinas. Nesse trabalho, três diferentes categorias de datas de entrega são consideradas: restritiva, muito restritiva e não restritiva. As datas muito restritivas fazem com que todas as tarefas do sistema fiquem atrasadas. As datas não restritivas não restringem o sequenciamento das tarefas. As datas restritivas são datas que ficam entre as datas muito restritivas e as não restritivas. Como solução inicial para a busca tabu, Chandra et al. (2009) propuseram uma heurística construtiva baseada em uma regra de despacho que considerava a máquina gargalo do sistema em conjunto com as datas de entrega para escolher a tarefa da sequência e determinar os instantes de início de processamento. Um total de 1200 instâncias foram geradas pelos autores e os resultados obtidos pelo método proposto comparados com as soluções ótimas obtidas nos casos onde a data de entrega era não restritiva ou no casos onde as instâncias eram de pequeno porte. No geral, a distância não foi mais do que $1.71 \%$ em relação aos ótimos. Fernandez-Viagas et al. (2016) propuseram uma heurística construtiva para o FSP com minimização da somatória dos adiantamentos e atrasos com datas de entrega diferentes. A heurística, a cada iteração, seleciona uma tarefa baseada em uma lista de prioridades dinâmica que considera os tempos ociosos, os instantes de término de processamento, os adiantamentos e os atrasos de cada tarefa. A heurística foi combinada com uma busca local iterativa e os resultados do método foram comparados com outras heurísticas que também utilizam lista de prioridade dinâmica.

Neste trabalho, o FSP com tempos de espera será abordado com a mesma metodologia utilizada no Capítulo 2. Inicialmente, um MIP para o FSP-WT é descrito na Seção 3.1. A Seção 3.2 propõe um método list scheduling que utiliza uma regra de seleção baseada no encaixe das tarefas nas janelas da programação. Além disso, a Seção 3.2 ainda descreve uma rotina para definir os instantes de início de processamento de uma dada sequência de tarefas. O método Beam Search é então definido 
na Seção 3.3. Os resultados computacionais obtidos pelos métodos propostos são reportados e discutidos na Seção 3.4. Por último, a Seção 3.5 apresenta as conclusões deste capítulo.

\subsection{Modelagem matemática}

O FSP-WT é definido como segue. Sejam $n$ tarefas para serem processadas em $m$ máquinas. Todas as tarefas possuem uma mesma data de entrega $d$ e devem passar em cada uma das máquinas numa mesma rota de processamento, quer dizer, toda tarefa $i, i=1, \ldots, n$, será processada pela $k$-ésima máquina, $k=1, \ldots, m$, com tempo de processamento $p_{i k}$, no seu $k$-ésimo estágio de processamento. Portanto, o problema se resume em definir instantes de início de processamento $s_{i k}$, ou equivalentemente definir instantes de término de processamento $C_{i k}$, para cada um dos estágios de todas as tarefas, garantindo que nenhum processamento se sobreponha e que a rota através das máquinas seja respeitada. Além disso, neste trabalho considera-se o problema com permutação de sequência, quer dizer, a sequência do processamento das tarefas deve ser a mesma em todas as máquinas. Uma vez iniciado o processamento de um estágio de uma tarefa, este processamento não pode ser interrompido e a capacidade de armazenamento entre as máquinas é infinita. O objetivo é a minimização dos adiantamentos $E_{i}=d-C_{i, m}$ e dos atrasos $T_{i}=C_{i, m}-d$. Em complemento, deseja-se escolher dentre as soluções que minimizam os adiantamentos e os atrasos, aquela que possui menor somatória dos tempos de espera entre os estágios de processamento. As equações (3.1)-(3.10) descrevem um MIP para o FSP-WT. As Tabelas 3.1 e 3.2 descrevem as variáveis e os parâmetros utilizados no MIP.

\begin{tabular}{ll}
\hline \multicolumn{1}{c}{ Variáveis } \\
\hline$E_{i}$ & Adiantamento da tarefa $i$. \\
$T_{i}$ & Atraso da tarefa $i$. \\
$C_{i k}$ & Instante de término de processamento da tarefa $i$ na máquina $k$. \\
$z_{i j}$ & 1 se o job $i$ precede o job $j$ na sequência. 0 caso contrário. \\
$W_{i k}$ & Tempo de espera da tarefa $i$ entre a máquina $k$ e $k+1$. \\
\hline
\end{tabular}

Tabela 3.1: Variáveis do modelo matemático do FSP-WT.

\begin{tabular}{ll}
\hline & \multicolumn{1}{c}{ Parâmetros } \\
\hline$d$ & Data de entrega das tarefas. \\
$p_{i k}$ & Tempo de processamento da tarefa $i$ na máquina $k$. \\
$P$ & Soma dos tempos de processamento calculada como $\sum_{i=1}^{n} \sum_{k=1}^{m} p_{i k}$ \\
$M$ & Constante positiva suficientemente grande. \\
\hline
\end{tabular}

Tabela 3.2: Parâmetros do modelo matemático do FSP-WT. 
Minimize $n(d+P)\left(\sum_{i=1}^{n}\left(E_{i}+T_{i}\right)\right)+\sum_{i=1}^{n} \sum_{k=1}^{m-1} W_{i k}$

Sujeito a:

$$
\begin{aligned}
T_{i} \geq C_{i m}-d, & i=1, \ldots, n, \\
E_{i} \geq d-C_{i m}, & i=1, \ldots, n, \\
C_{i 1} \geq p_{i 1}, & i=1, \ldots, n, \\
C_{i, k+1}=p_{i, k+1}+C_{i k}+W_{i k}, & i=1, \ldots, n ; k=1, \ldots, m-1, \\
C_{i k} \geq p_{i k}+C_{j k}-M z_{i j}, & i=1, \ldots, n ; j=i+1, \ldots, n, k=1, \ldots, m \\
C_{j k} \geq p_{j k}+C_{i k}-M\left(1-z_{i j}\right), & i=1, \ldots, n ; j=i+1, \ldots, n, k=1, \ldots, m, \\
z_{i j} \in\{0,1\}, & i=1, \ldots, n ; j=i+1, \ldots, n, \\
T_{i}, E_{i} \geq 0, & i=1, \ldots, n, \\
W_{i k} \geq 0, & i=1, \ldots, n ; k=1, \ldots, m-1 .
\end{aligned}
$$

A função objetivo (3.1) relaciona a soma dos adiantamentos e dos atrasos com os tempos de espera das tarefas entre as máquinas. Nela, a constante $n(d+P)$ é um limitante superior da soma dos tempos de espera para o conjunto de soluções que minimizam a soma dos adiantamentos e dos atrasos. A constante garante que o modelo nos leve à soluções que minimizam o tempo de espera das tarefas entre as máquinas $\left(\sum_{i=1}^{n} \sum_{k=1}^{m-1} W_{i k}\right)$ dentre aquelas que minimizam os adiantamentos e atrasos $\left(\sum_{i=1}^{n} E_{i}+T_{i}\right)$. As restrições (3.2) e (3.3) calculam os adiantamentos e os atrasos para cada tarefa. A restrição (3.4) garante que o primeiro estágio de processamento de cada tarefa seja processado por pelo menos o seu tempo de processamento. A restrição (3.5) relaciona os tempos de espera de cada tarefa com os instantes de término de processamento e os tempos de processamento. Finalmente, as restrições (3.6) e (3.7) garantem que as tarefas sejam processadas pelas máquinas na ordem estabelecida pela variável binária $z$ e que os processamentos não se sobreponham.

Para entender o limitante superior $n(d+P)$, primeiramente considere a restrição (3.5). Com ela, é fácil ver que o tempo de espera de uma tarefa $i$ entre a máquina $k$ e a máquina $k+1$ é dado por $W_{i k}=C_{i, k+1}-C_{i k}-p_{i k}$. Com isso, a soma dos tempos de espera do sistema pode ser escrita como $\sum_{i=1}^{n} \sum_{j=1}^{m-1} C_{i, k+1}-C_{i k}-p_{i k}$ ou de forma equivalente como

$$
\sum_{i=1}^{n}\left(C_{i m}-C_{i 1}\right)-\sum_{i=1}^{n} \sum_{k=2}^{m} p_{i k} .
$$

Uma vez que $\sum_{i=1}^{n} \sum_{k=2}^{m} p_{i k}$ é uma constante, minimizar a soma dos tempos de espera é o mesmo que minimizar

$$
\sum_{i=1}^{n}\left(C_{i m}-C_{i 1}\right)
$$

Assumindo que os dados do problema, quer dizer, os tempos de processamento $p_{i k}$ e a data de entrega $d$, são valores inteiros, então toda solução ótima para o problema de somente minimizar os adiantamentos e atrasos é tal que $E_{i}$ e $T_{i}$ também são valores inteiros e portanto a soma $\sum_{i=1}^{n}\left(E_{i}+T_{i}\right)$ também é um valor inteiro. Assim, dado qualquer solução ótima do problema de somente minimizar os adiantamentos e atrasos, é verdade que

$$
\sum_{i=1}^{n} C_{i m}<n(d+P),
$$

Demonstração. Considere uma solução viável para o problema de minimização dos adiantamentos 
e atrasos $E_{i}$ e $T_{i}$ tal que as tarefas são processadas na mesma ordenação de seus índices. A tarefa 1 inicia processamento no instante $d$ e possui tempo de término de processamento $C_{1 m}=d+\sum_{k=1}^{m} p_{1 k}$ e para as demais tarefas $i=2, \ldots, n$, a tarefa $i$ inicia processamento no instante $C_{i-1, m}$ e possui tempo de término de processamento no instante $C_{i m}=C_{i-1, m}+\sum_{k=1}^{m} p_{i k}$. Nesta solução viável, $\sum_{i=1}^{n} C_{i m}=n d+\sum_{i=1}^{n}\left((n-i+1) \sum_{k=1}^{m} p_{i k}\right)<n d+n \sum_{i=1}^{n} \sum_{k=1}^{m} p_{i k}=n(d+P)$.

Portanto, de (3.11) temos

$$
\sum_{i=1}^{n}\left(C_{i m}-C_{i 1}\right) \leq \sum_{i=1}^{n} C_{i m}<n(d+P),
$$

ou, equivalentemente

$$
\frac{\sum_{i=1}^{n}\left(C_{i m}-C_{i 1}\right)}{n(d+P)}<1 .
$$

Assim, somar a fração do lado esquerdo de (3.12) na função objetivo do problema de somente minimizar a soma dos adiantamentos e atrasos é inócuo. Além disso, a minimização da função objetivo que combina a soma dos adiantamentos e atrasos e o lado esquerdo de (3.12) tem o efeito de dentre as soluções que minimizam os adiantamentos e os atrasos escolher aquela com menor soma dos tempos de espera.

\subsection{Algoritmo List Scheduling}

O algoritmo list scheduling (LS) para o FSP-WT deste trabalho consiste em um algoritmo heurístico que constrói a sequência em que as tarefas devem ser programadas nas máquinas baseado em uma lista dinâmica de prioridades. Em palavras, a filosofia do algoritmo é a de priorizar as tarefas que preenchem da melhor maneira possível as janelas deixadas pela programação das tarefas anteriores.

Para entender o significado de "janela", suponha um cenário com $n=3$ tarefas e $m=2$ máquinas. Os tempos de processamento são dados de acordo com a Tabela 3.3. A Figura 3.1 ilustra a janela entre as máquinas $M_{1}$ e $M_{2}$ provocada pela programação da tarefa $J_{1}$.

\begin{tabular}{|c|cc|}
\hline \multirow{2}{*}{ Tarefas } & \multicolumn{2}{|c|}{ Máquinas } \\
\cline { 2 - 3 } & $M_{1}$ & $M_{2}$ \\
\hline \hline$J_{1}$ & 5 & 5 \\
$J_{2}$ & 2 & 2 \\
$J_{3}$ & 3 & 2 \\
\hline
\end{tabular}

Tabela 3.3: Tempos de processamento para um problema com $n=3$ tarefas e $m=2$ máquinas.

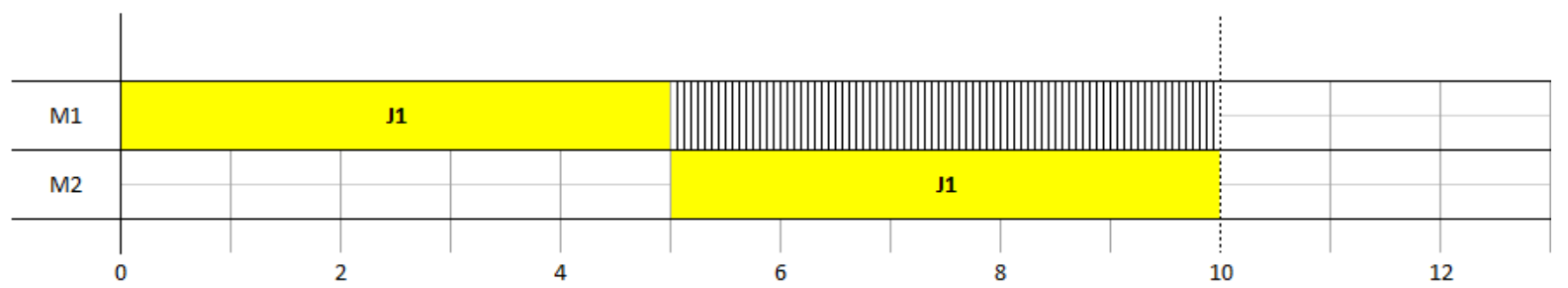

Figura 3.1: Programação da tarefa $J_{1}$ nas máquinas $M_{1}$ e $M_{2}$ de acordo com o problema descrito na Tabela 3.3. A parte hachurada representa a janela provocada pela programação.

A filosofia do LS proposto vai de encontro com as características do problema, visto que os tempos de espera das tarefas entre as máquinas estão diretamente relacionados com as janelas 
provocadas pela programação da sequência. Nesta linha, em uma programação ideal, todas as janelas seriam totalmente preenchidas e não haveriam tempos de espera. As Figuras 3.2 e 3.3 ilustram respectivamente a programação das tarefas $J_{2}$ e $J_{3}$ após $J_{1}$ para o exemplo descrito na Tabela 3.3. Nas figuras, a parte hachurada é a porção não preenchida da janela de $J_{1}$. Comparando as programações, note que escolher a tarefa $J_{3}$ ao invés da tarefa $J_{2}$ como a próxima tarefa da sequência produz uma programação que preenche melhor a janela e também com menores tempos de espera, quer dizer, $J_{3}$ se "encaixa" melhor do que $J_{2}$. Suponha que então a tarefa $J_{3}$ seja escolhida para seguir a tarefa $J_{1}$ na programação. A Figura 3.4 mostra a janela atualizada, dada a programação da sequência $J_{1}, J_{3}$.

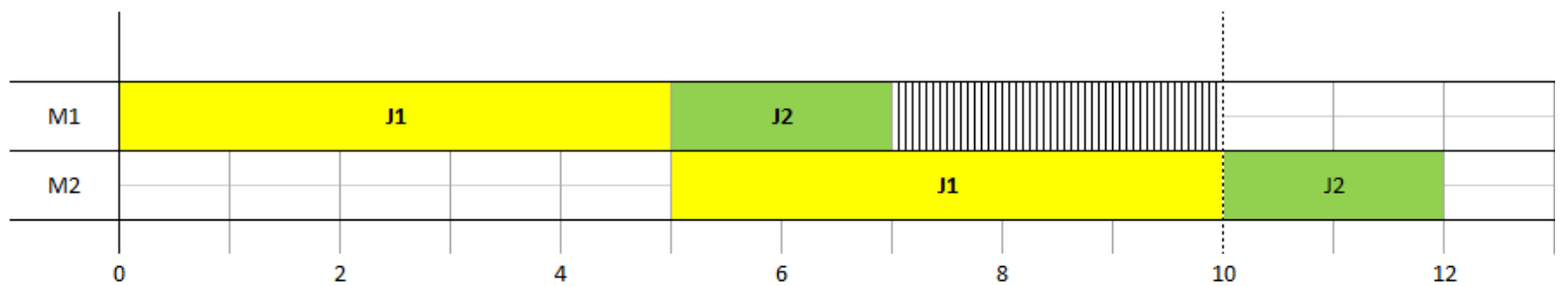

Figura 3.2: Programação das tarefas $J_{1}$ e $J_{2}$ nas máquinas $M_{1}$ e $M_{2}$ de acordo com o problema descrito na Tabela 3.3. A parte hachurada representa o restante da janela de $J_{1}$.

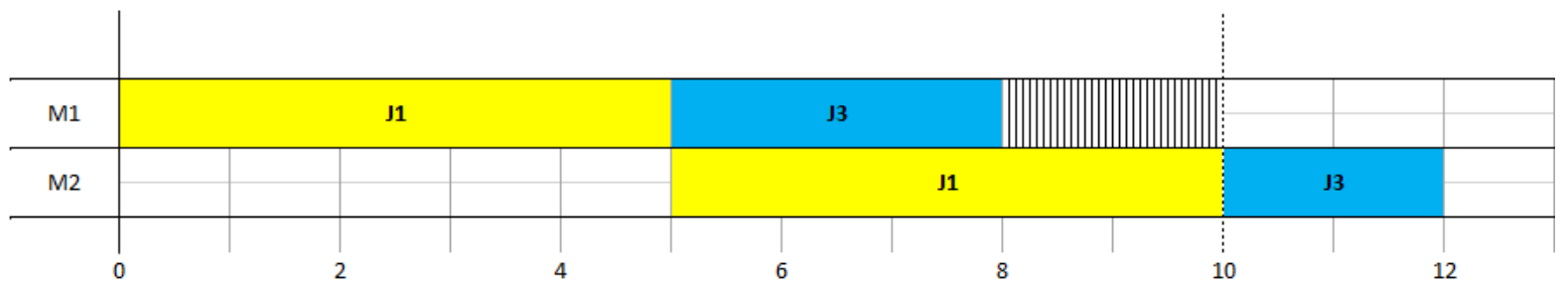

Figura 3.3: Programação das tarefas $J_{1}$ e $J_{3}$ nas máquinas $M_{1}$ e $M_{2}$ de acordo com o problema descrito na Tabela 3.3. A parte hachurada representa o restante da janela de $J_{1}$.

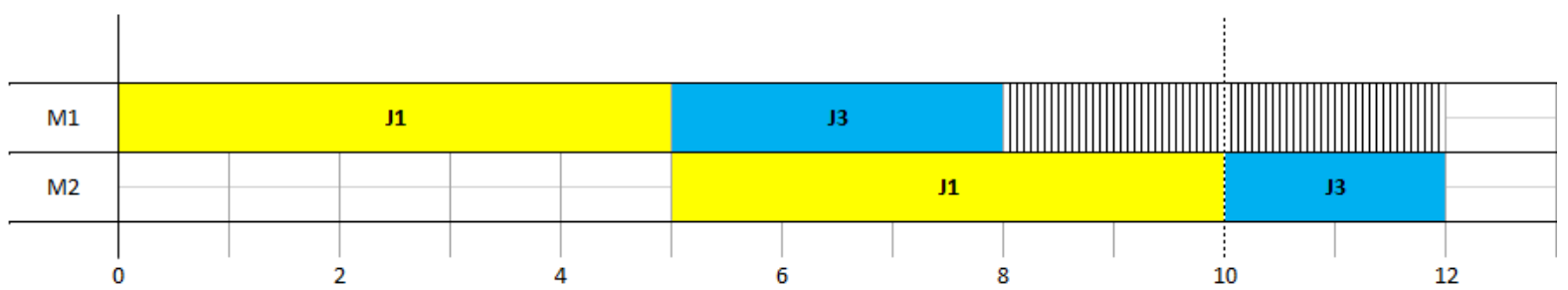

Figura 3.4: Programação das tarefas $J_{1}$ e $J_{3}$ nas máquinas $M_{1}$ e $M_{2}$ de acordo com o problema descrito na Tabela 3.3. A parte hachurada representa o restante da janela de $J_{1}$.

A Figura 3.5 ilustra a programação de $J_{2}$ e $J_{3}$ nas máquinas $M_{1}$ e $M_{2}$ de acordo com o problema descrito na Tabela 3.3. É possível ver na figura que na máquina $M_{1}$, a janela de $J_{2}$ é preenchida com sobra por $J_{3}$. Em um cenário onde a data de entrega fosse maior que 4 para este problema, essa sobra seria indesejada visto que ela provocaria tempo de espera entre as máquinas para $J_{2}$.

Duas programações ótimas possíveis para a soma dos adiantamentos e dos atrasos considerando o problema da Tabela 3.3 e data de entrega comum $d=10$ são ilustradas pela Figura 3.6. Nas programações, a soma dos adiantamentos e dos atrasos valem $\sum_{i=1}^{n} E_{i}+T_{i}=0+0+0+0+2+4=6$. Para a primeira programação (programação de cima), as somas dos tempos de espera entre as 


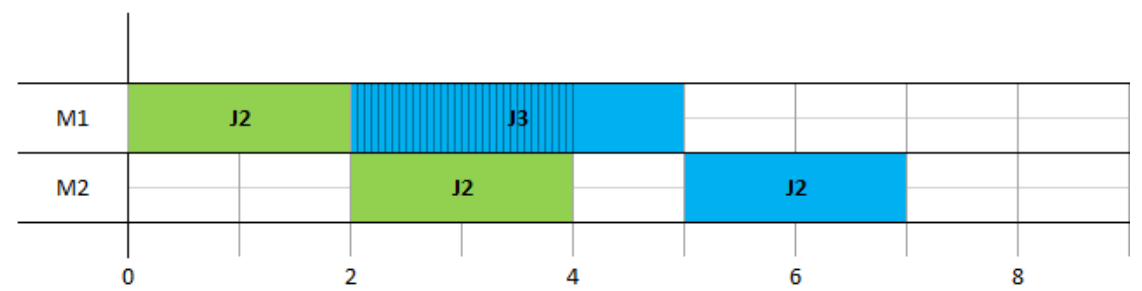

Figura 3.5: Programação das tarefas $J_{2}$ e $J_{3}$ nas máquinas $M_{1}$ e $M_{2}$ de acordo com o problema descrito na Tabela 3.3. A parte hachurada representa a janela de $J_{2}$.

máquinas valem $\sum_{k=1}^{m-1} W_{i k}=0+3+2=5$. Na segunda programação (programação de baixo), a soma dos tempos de espera entre as máquinas valem $\sum_{k=1}^{m-1} W_{i k}=0+1+0=1$. Note que é possível chegar na segunda programação se simplesmente as tarefas $J_{2}$ e $J_{3}$ forem empurradas dois instantes de tempo na primeira máquina.

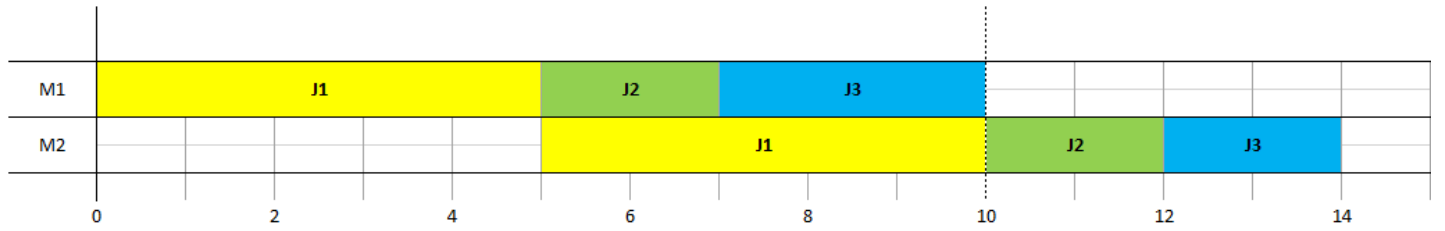

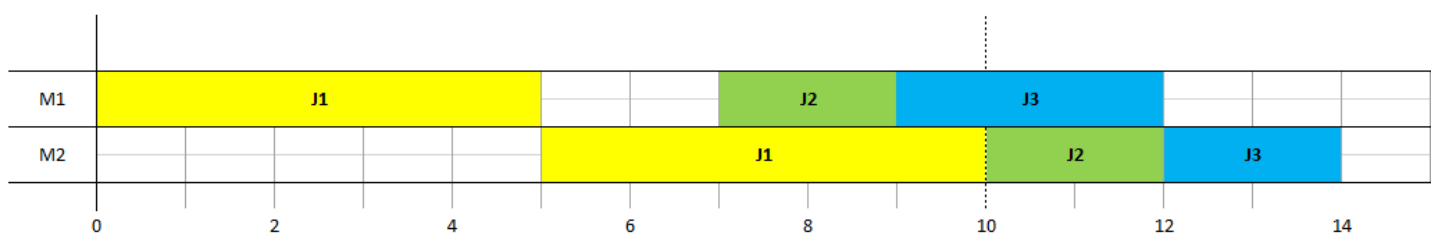

Figura 3.6: Duas programações possiveis considerando o cenário descrito na Tabela 3.3 e data de entrega comum $d=10$. A programação de baixo é o resultado de empurrar as tarefas $J_{2}$ e $J_{3}$ dois instantes de tempo na programação de cima. Este processo mantêm as somas dos adiantamentos e dos atrasos mas diminui a soma dos tempos de espera.

O Algoritmo 6 descreve o LS proposto nesta seção. Nas linhas 2-5, o algoritmo calcula a somatória dos tempos de processamentos das tarefas e escolhe a tarefa com menor somatória para ser a primeira tarefa da sequência. As linhas 6-9 faz a programação da então sequência parcial com uma única tarefa, escolhendo os menores instantes de términos de processamento possíveis. As linhas 10-23 descrevem o laço principal do método. A cada iteração, o método calcula as janelas da programação (linha 11) e para cada tarefa ainda não escolhida, calcula os encaixes nas janelas (linha 15). Para escolher a próxima tarefa a fazer parte da sequência, o LS utiliza uma estimativa que considera os encaixes e a somatória dos tempos de processamento de cada tarefa candidata e depois seleciona aquela com menor estimativa e caso haja empates, o algoritmo utiliza o menor índice das tarefas como critério de desempate (linhas 17-18). Uma vez que a escolha é feita, a sequência e a programação são então atualizadas (linhas 18-22). O método escolhe uma tarefa para o sequenciamento por iteração. No final das iterações do laço principal, todas as $n$ tarefas estarão no sequenciamento.

É importante dizer que o LS descrito pelo Algoritmo 6 apenas calcula uma sequência e a programação utilizada no processo é descartada. A programação final é calculada por uma versão estendida para $m$ máquinas do algoritmo timing apresentado em Sakuraba et al. (2009). O algoritmo timing foi inicialmente proposto por Hendel e Sourd (2007) para um problema de minimização dos adiantamentos e atrasos com máquina única e datas de liberação. Datas de liberação significam que as tarefas só podem iniciar processamento na máquina após um determinado instante de tempo $r_{i}, i=1, \ldots, n$. O algoritmo retorna a programação ótima para qualquer sequência dada conside- 


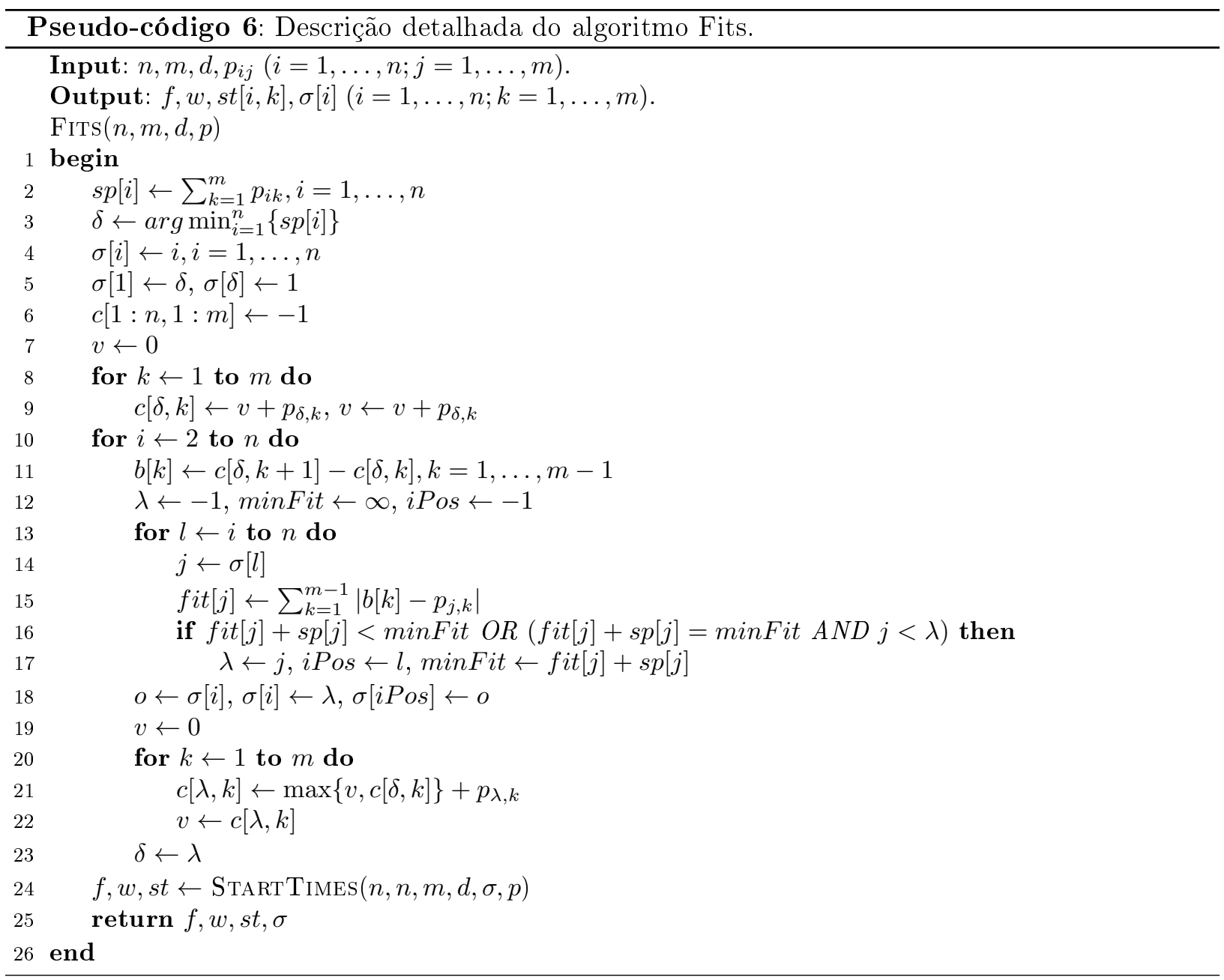

rando o problema de minimizar as somas dos adiantamentos e dos atrasos (Hendel e Sourd, 2007; Sakuraba et al., 2009). Sakuraba et al. (2009) estenderam o algoritmo para o problema com 2 máquinas programando o mais cedo possível as tarefas na primeira máquina e utilizando os instantes de término de processamento na primeira como datas de liberação para a segunda máquina e então aplicando o algoritmo de timing original. Neste trabalho, dado uma sequência qualquer, o cálculo da programação é feito por uma extensão natural da ideia de Sakuraba et al. (2009) para $m$ máquinas onde a programação nas $m-1$ primeiras máquinas é feita o mais cedo possível e depois o algoritmo timing calcula a programação ótima para a máquina $m$ utilizando os instantes de término de processamento na máquina $m-1$ como instantes de liberação. O pseudocódigo para esta extensão é descrito pelo Algoritmo 7. O algoritmo recebe os parâmetros $\lambda, n, m, d, \sigma, p$ como entrada onde $\lambda$ é a quantidade de tarefas na sequência $\sigma, n, m$ e $d$ são o número de tarefas, número de máquinas e data de entrega comum respectivamente. $\sigma$ é a sequência de tarefas e $p$ é a matriz de tempos de processamento. No pseudocódigo, as linhas 2-10 calculam os menores instantes de início de processamento para as tarefas em $\sigma$ nas $m-1$ primeiras máquinas. O algoritmo Timing calcula então os instantes de início de processamento para a máquina $m$ na linha 11 . As linhas $12-17$ empurram as tarefas nas $m-1$ primeiras máquinas, como no exemplo da Figura 3.6. A soma dos tempos de espera é então calculada nas linhas 18-21. Por fim, a soma dos adiantamentos e dos atrasos para cada tarefa é calculada nas linhas 22-24.

Portanto, dado uma sequência de tarefas qualquer, o algoritmo STARTTimes (Algoritmo 7) calcula os inícios de processamento ótimos na última máquina para o problema de minimização dos adiantamentos e dos atrasos e empurra a programação nas $m-1$ primeiras máquinas para possivelmente reduzir os tempos de espera. Contudo, este processo não é ótimo para o FSP-WT. Esta afirmação é demonstrada na Figura 3.7. A figura apresenta três programações ótimas para 


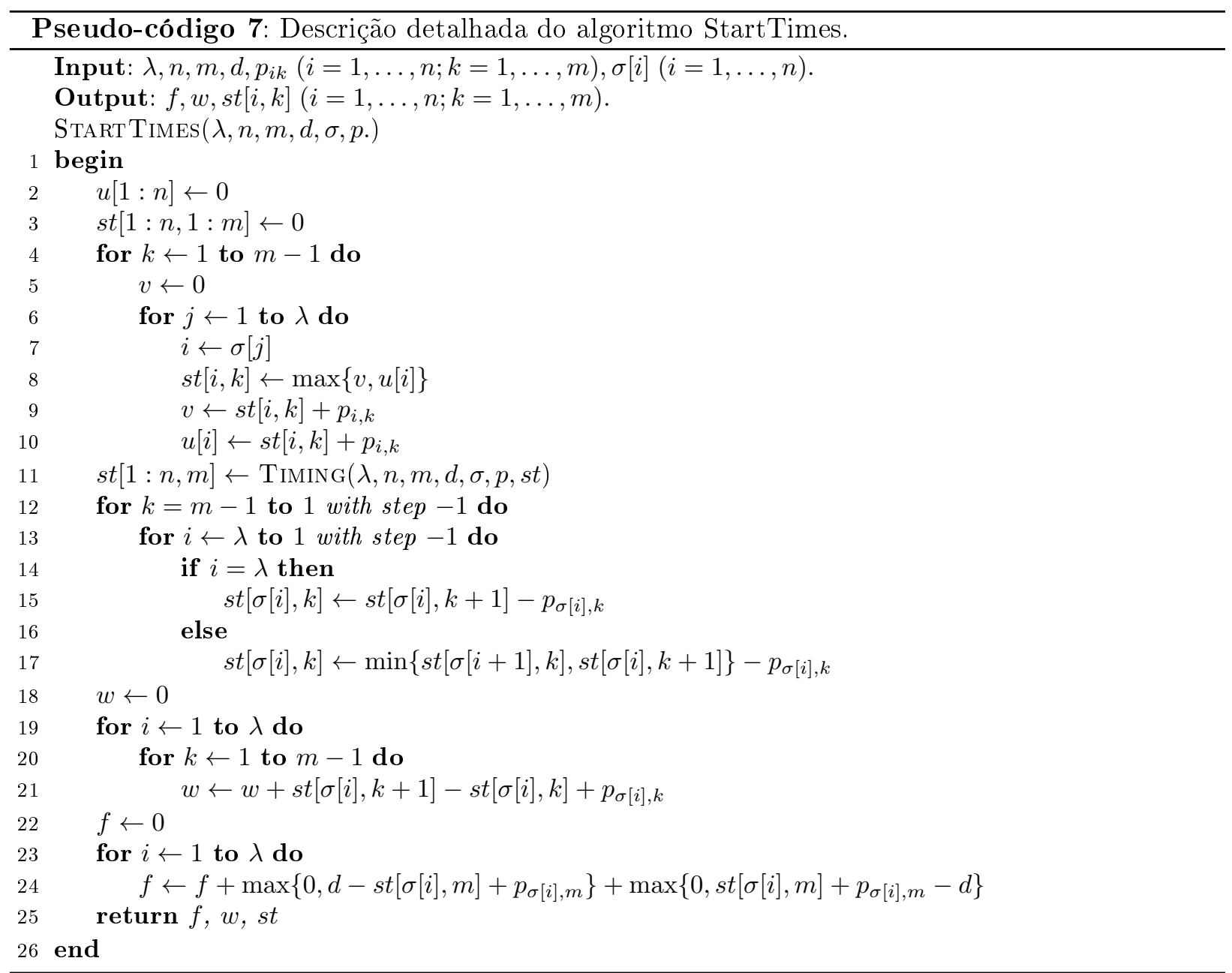

a soma dos adiantamentos e atrasos para o problema descrito na Tabela $3.4 \mathrm{com}$ data de entrega comum $d=7$. A programação $(a)$ é o resultado da chamada do Algoritmo $7 \mathrm{com}$ a sequência $J_{1}, J_{2}, J_{3}$ sem o processo de empurrar (linhas 12-14) e possui soma dos adiantamentos e atrasos igual a 4 e soma dos tempos de espera igual a 2 . A programação (b) é o resultado da chamada com o processo de empurrar e possui soma dos adiantamentos e atrasos e soma dos tempos de espera também iguais a 4 e 2 respectivamente. A programação $(c)$ é a programação ótima para o cenário descrito considerando o FPS-WT e possui soma dos adiantamentos e atrasos igual a 4 mas soma dos tempos de espera igual a 0 .

Contudo, diferentes sequências podem gerar diferentes programações com o mesmo valor de soma de adiantamentos e atrasos mas diferentes valores para os tempos de espera. A Figura 3.8 ilustra isso com três programações para o cenário descrito pela Tabela 3.5 com data de entrega comum $d=7$. Na figura, a programação (a) é a programação da sequência $J_{1}, J_{3}, J_{2}$ com os instantes de início de processamento calculados pelo Algoritmo 7 sem o processo de empurrar. Em (a), a soma dos adiantamentos e atrasos é igual a 2 e a soma dos tempos de espera é igual a 3 . A programação (b) é a programação da sequência $J_{1}, J_{3}, J_{2}$ com os instantes de início de processamento calculados pelo Algoritmo 7 com o processo de empurrar. Em (b), a soma dos adiantamentos e atrasos é igual a 2 e a soma dos tempos de espera é igual a 1. A programação (c) é a programação da sequência $J_{1}, J_{2}, J_{3}$ com os instantes de início de processamento calculados pelo Algoritmo 7 com o processo de empurrar. Em (c), a soma dos adiantamentos e atrasos é igual a 2 e a soma dos tempos de espera é igual a 0 . 


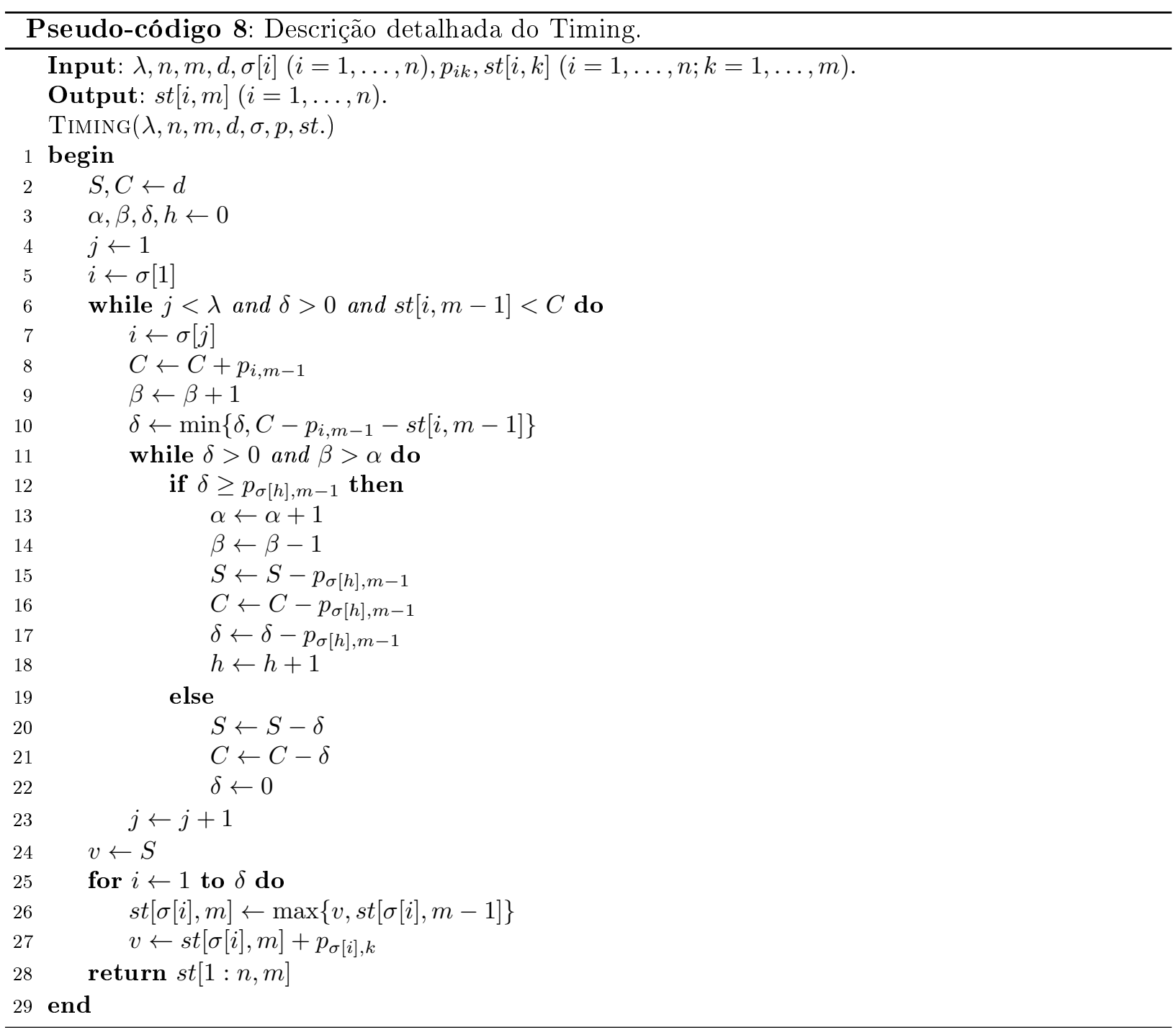

\begin{tabular}{|c|ccc|}
\hline \multirow{2}{*}{ Tarefas } & \multicolumn{3}{|c|}{ Máquinas } \\
\cline { 2 - 4 } & $M_{1}$ & $M_{2}$ & $M_{3}$ \\
\hline \hline$J_{1}$ & 1 & 2 & 3 \\
$J_{2}$ & 3 & 2 & 1 \\
$J_{3}$ & 3 & 1 & 2 \\
\hline
\end{tabular}

Tabela 3.4: Tempos de processamento para um problema com $n=3$ tarefas e $m=3$ máquinas.

\begin{tabular}{|c|ccc|}
\hline \multirow{2}{*}{ Tarefas } & \multicolumn{3}{|c|}{ Máquinas } \\
\cline { 2 - 4 } & $M_{1}$ & $M_{2}$ & $M_{3}$ \\
\hline \hline$J_{1}$ & 1 & 2 & 3 \\
$J_{2}$ & 2 & 1 & 1 \\
$J_{3}$ & 1 & 2 & 1 \\
\hline
\end{tabular}

Tabela 3.5: Tempos de processamento para um problema com $n=3$ tarefas e $m=3$ máquinas. 
(a)

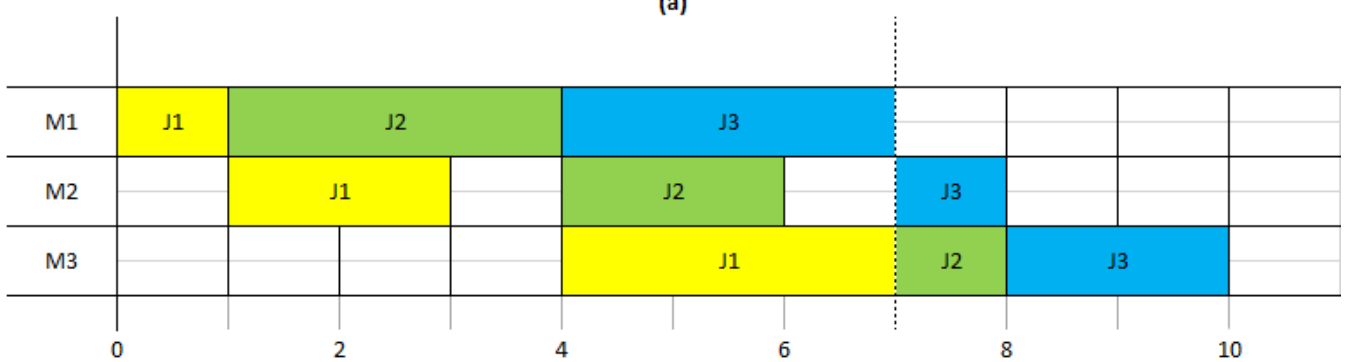

(b)

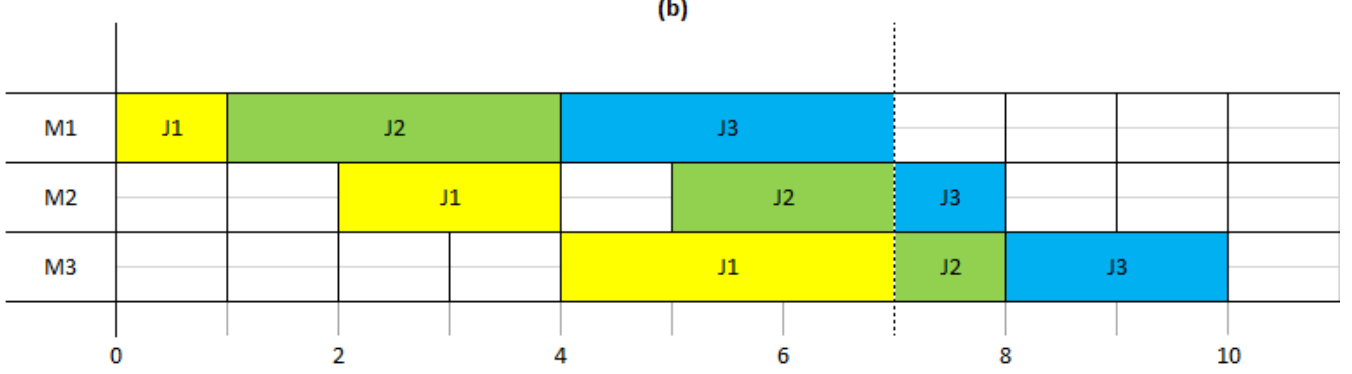

(c)

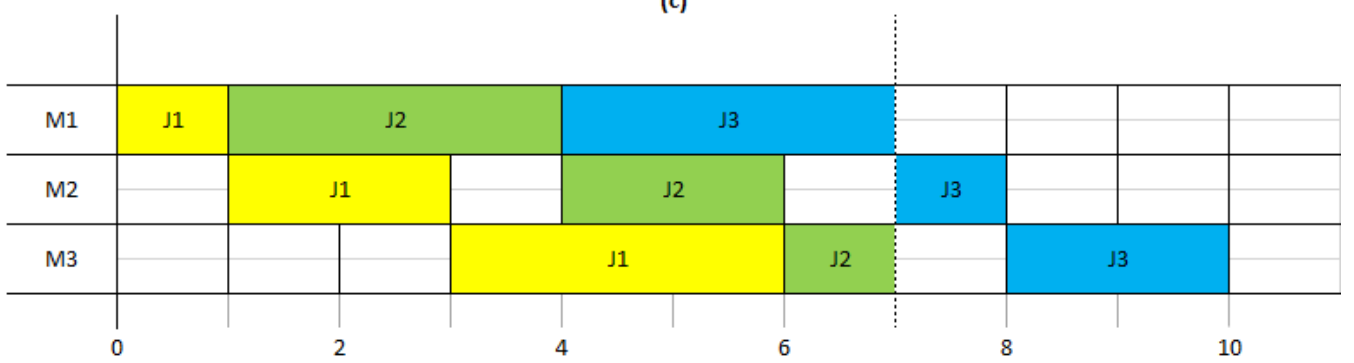

Figura 3.7: Três programações possiveis para a sequência de tarefas $J_{1}, J_{2}, J_{3}$ considerando o cenário descrito na Tabela 3.4 e data de entrega comum $d=7$. A programação (a) é o resultado da chamada do Algoritmo 7 sem o processo de empurrar. A programação (b) é o resultado da chamada com o processo de empurrar. A programação (c) é a programação ótima para o FSP-WT.

\subsection{Método Beam Search}

O método Beam Search (BS) para o FSP-WT neste trabalho foi desenvolvido utilizando a mesma metodologia do método BS para o FJSP-SF do Capítulo 2. Assim, como no capítulo anterior, ele também é uma extensão natural do LS apresentado na Seção 3.2 que explora várias tarefas a cada iteração ao invés de somente uma.

Nesta linha, o método BS aqui descrito realiza podas na árvore de busca utilizando dois mecanismos heurísticos: a busca local e a busca global. A busca local consiste em uma avaliação rápida e local dos nós da árvore de busca, enquanto a busca global é uma avaliação mais profunda e consequentemente, mais demorada. A busca local tem o papel de filtrar as possibilidades e escolher quais os $\alpha$ nós da árvore valem a pena serem explorados pela busca global. A busca global determina quais os $\beta$ nós da árvore vão permanecer para a próxima iteração do método. Uma vez que o método utiliza o mecanismo de filtro de soluções, ele também é conhecido como filtered Beam Search.

Portanto, são três os principais processos do filtered BS: a expansão dos níveis, a busca local e a busca global. As Figuras 3.9 e 3.10 ilustram o funcionamento destes três processos na expansão do nível 1 para o nível 2 utilizando $\alpha=\beta=2$. A Figura 3.9 mostra todos os possíveis nós de cada pai, ou seja, a expansão do nível. Na Figura 3.10 a busca local do método é aplicada, avaliando os filhos do primeiro pai em 5, 8 e 3 respectivamente e os filhos do segundo pai em 1, 9 e 6 respectivamente. Os filhos hachurados são os nós escolhidos pela busca local para serem avaliados pela busca global. Note que somente 2 dos 3 filhos de cada pai foram escolhidos. Por fim, na Figura 3.11 a busca global 
(a)

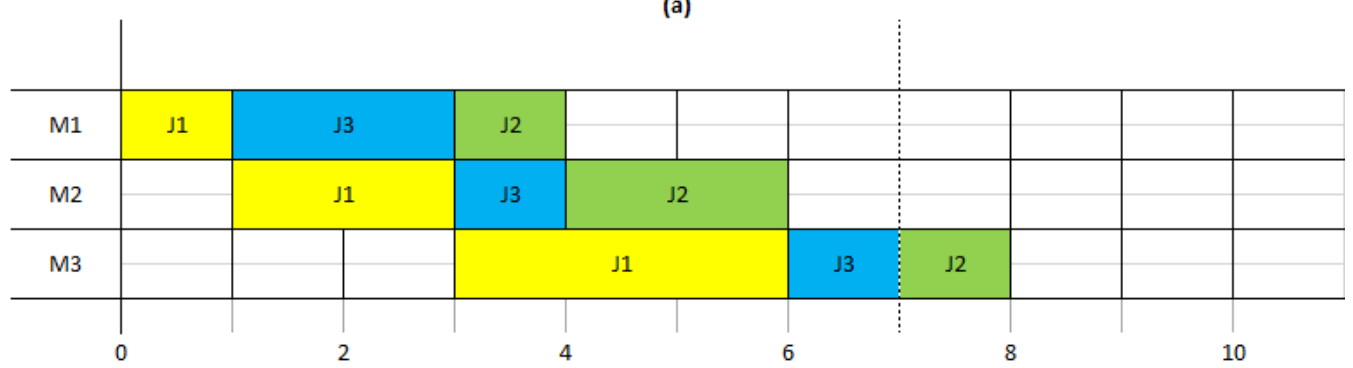

(b)

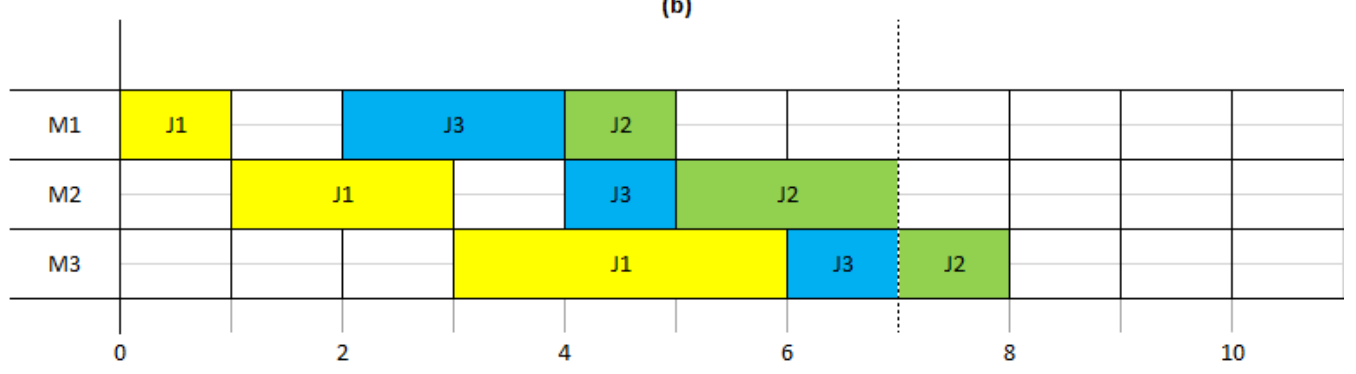

(c)

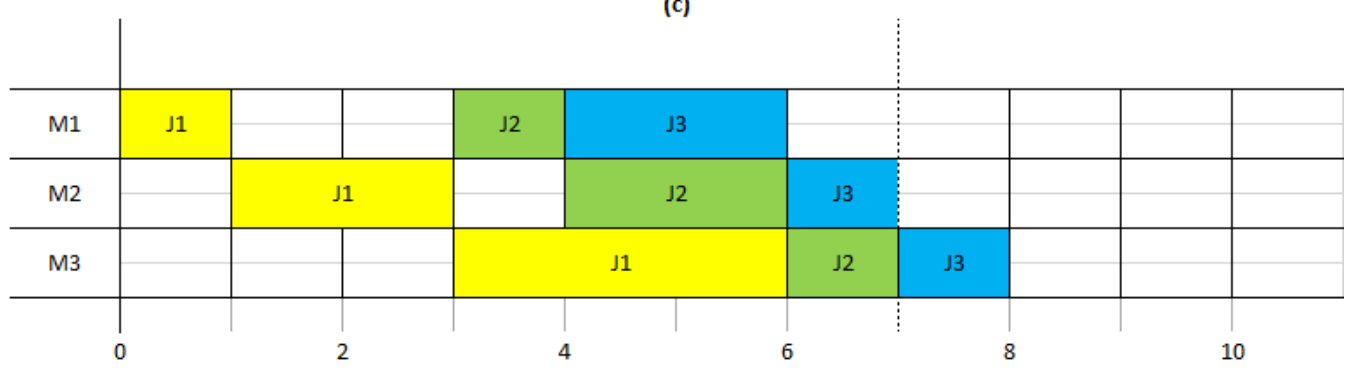

Figura 3.8: Três programações ótimas para a soma dos adiantamentos e atrasos considerando o cenário descrito na Tabela 3.5 e data de entrega comum $d=7$ mas com diferentes soma dos tempos de espera. A programação (a) é o resultado da programação da sequência $J_{1}, J_{3}, J_{2}$ utilizando o Algoritmo 7 sem o processo de empurrar. A programação (b) é o resultado da programação da sequência $J_{1}, J_{3}, J_{2}$ utilizando o Algoritmo 7 com o processo de empurrar. A programação (c) é o resultado da programação da sequência $J_{1}, J_{2}, J_{3}$ utilizando o Algoritmo 7 com o processo de empurrar. (c) é ótimo para o FSP-WT.

avalia os filhos em 1, 2, 1 e 3 respectivamente e seleciona somente os dois melhores candidatos para a próxima iteração do método.

Cada nó na árvore do BS proposto representa uma solução parcial para o FSP-WT. Além disso, um nó do nível $\ell$ da árvore já possui $\ell$ tarefas escolhidas no sequenciamento que ele representa. Portanto, cada nó é formado pela tupla $(i, \lambda, f, w, \sigma, \phi)$ onde

$i$ A última tarefa que foi adicionada na sequência parcial que o nó representa.

$\boldsymbol{\lambda}$ Quantidade de tarefas já escolhidas para o sequenciamento ou equivalentemente, o nível que o nó pertence.

$f$ Valor da soma dos adiantamentos e dos atrasos do sequenciamento representado pelo nó e calculado com o uso do Algoritmo 7.

$\boldsymbol{w}$ Valor da soma dos tempos de espera do sequenciamento representado pelo nó e calculado com o uso do Algoritmo 7.

$\boldsymbol{\sigma}$ Vetor de inteiros de tamanho $n$ com as $\lambda$ primeiras posições preenchidas com valores entre $[1, n]$ que guarda o sequenciamento das tarefas representado pelo nó. 


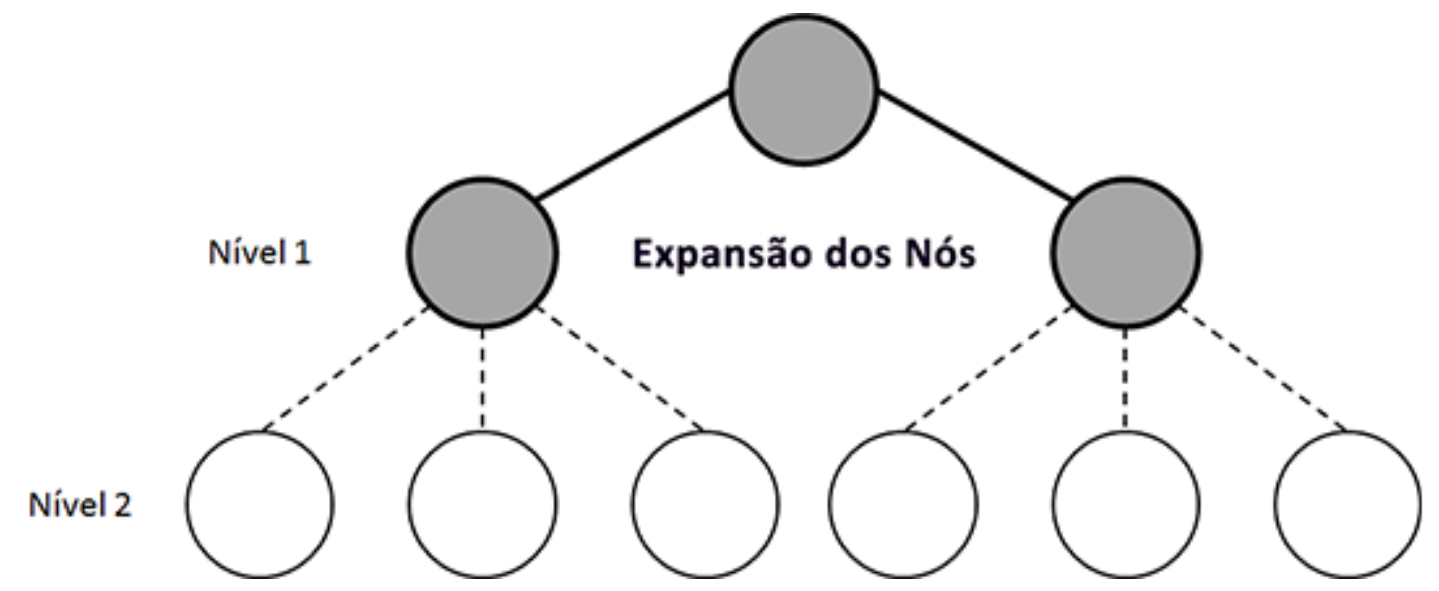

Figura 3.9: Esquema da expansão do nível 1 para o nível 2 na árvore de busca do BS. Cada nó pai possui três filhos candidatos para a expansão.

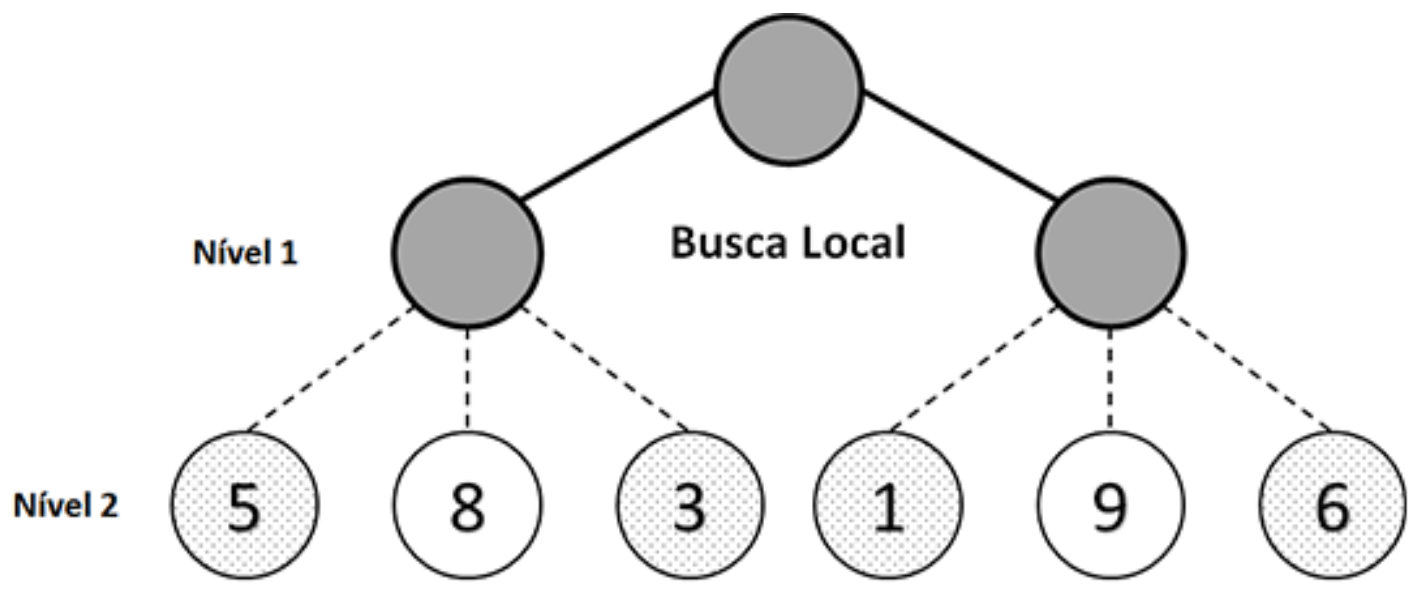

Figura 3.10: Aplicação da busca local com $\alpha=2$ no esquema ilustrado pela Figura 3.9. Cada nó pai possui três filhos candidatos para a expansão. Os filhos candidatos são avaliados pela busca local e somente os dois melhores (nós hachurados) são escolhidos para serem avaliados pela busca global.

$\phi$ vetor binário de tamanho $n$ que para cada posição $j$ do vetor, com $j=1, \ldots, n, \phi[j]=$ true caso a tarefa $j$ já tenha sido escolhida para o sequenciamento e está em alguma posição de $\sigma$ ou $\phi=$ false caso contrário.

Neste trabalho, a busca local proposta para o BS recebe um nó $(i, \lambda, f, w, \sigma, \phi)$ e avalia as tarefas candidatas, de acordo com os critérios de seleção de tarefas do LS da seção anterior que se baseia na janela da programação do sequenciamento parcial, no encaixe das tarefas candidatas e nas somas dos tempos de processamento. A busca global recebe um nó $(i, \lambda, f, w, \sigma, \phi)$ completa o sequenciamento parcial primeiramente com a tarefa candidata e em seguida utilizando o LS da seção anterior e avalia a sequência completa utilizando a soma dos adiantamentos, dos atrasos e dos tempos de espera são calculados pelo Algoritmo 7.

O Algoritmo 9 apresenta o pseudocódigo do BS para o FSP-WT. O pseudocódigo recebe $\eta, \kappa, \alpha, \beta, n, m, d$ e $p$ como parâmetros sendo $\eta$ e $\kappa$ parâmetros que influenciam a expansão dos primeiros níveis, que será discutida mais tarde, $\alpha$ e $\beta$ representam parâmetros da busca local e da busca global, respectivamente. $n, m, d$ e $p$ são a quantidade de tarefas, a quantidade de máquinas, a data de entrega comum e o tempo de processamento de cada tarefa em cada máquina. A quantidade de nós que serão mantidos e explorados a cada nível da árvore é calculada na linha 2. A quantidade de nós candidatos que serão escolhidos pela busca local para serem avaliados pela busca local é 


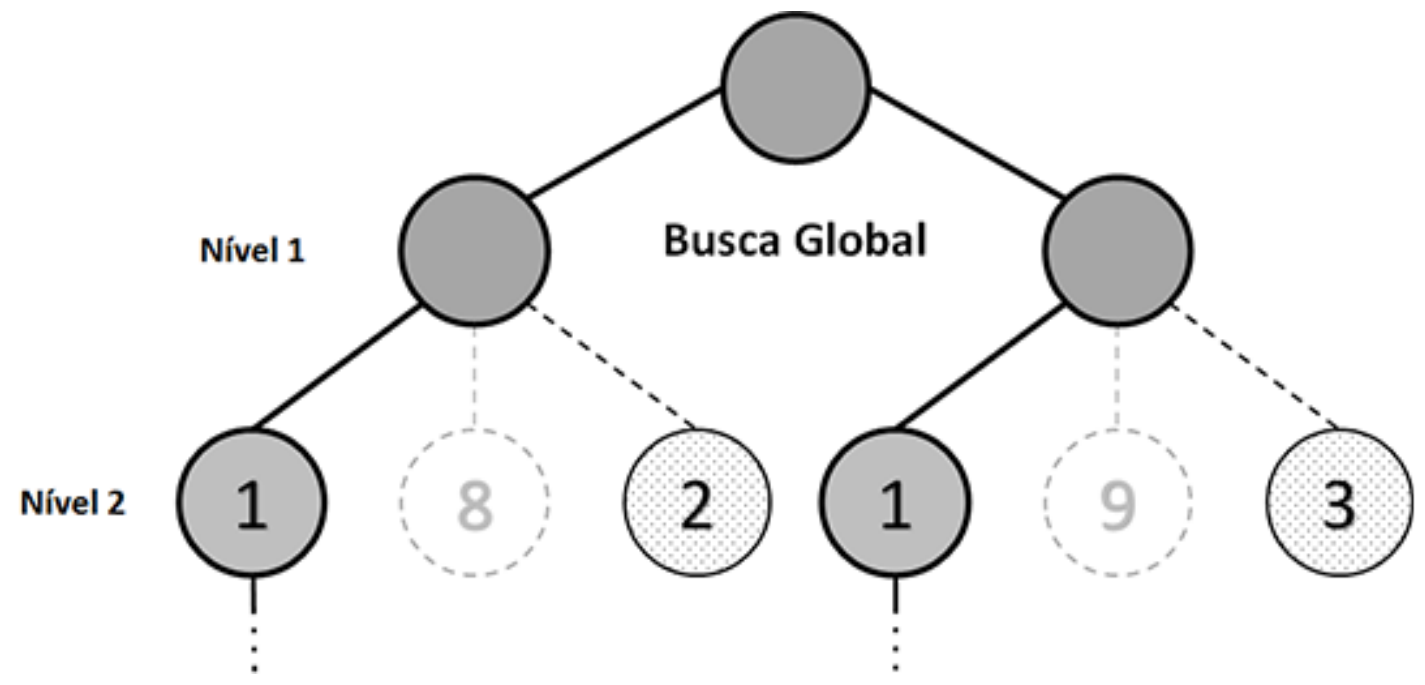

Figura 3.11: Aplicação da busca global com $\beta=2$ no esquema ilustrado pela Figura 3.10. Cada nó pai possui três filhos candidatos para a expansão. Os filhos candidatos escolhidos pela busca local são avaliados pela busca global e somente os dois melhores (nós coloridos) são mantidos na árvore para a próxima iteração do método.

calculada na linha 3. Note que ambos os valores são calculados em função da quantidade de tarefas $n$. No presente trabalho, adota-se que $\alpha, \beta \in(0,1]$. A linha 4 realiza a expansão dos primeiros $\eta$ níveis da árvore, quer dizer, após a execução da linha 4 , a árvore possuirá $\ddot{\beta}$ nós que representam um sequenciamento parcial com $\eta$ tarefas já escolhidas. A expansão dos primeiros níveis, como discutido no Capítulo 2, é extremamente importante para o sucesso do método. Em linhas gerais, nos primeiros níveis da árvore, o método aplica a busca global em todos os nós candidatos e mantêm $\kappa$ candidatos para o próximo nível. Ao atingir o $\eta$-ésimo nível, o método seleciona os $\ddot{\beta}$ melhores nós, de acordo com a busca global, e entra no laço principal da linha 5. O laço principal itera por $n-\eta$ vezes e para cada nó da árvore, a cada iteração, escolhe uma nova tarefa para fazer parte do seu sequenciamento. A variável $\mathcal{N}$ da linha 4 , é um conjunto que guarda os nós ativos da árvore. No laço principal, a linha 6 faz a expansão de cada nó em $\mathcal{N}$ e aplica a busca local em cada um dos candidatos escolhendo somente $\ddot{\alpha}$ para serem avaliados pela busca global. $\mathcal{B}$ é um conjunto de $\mathcal{N}$ 's e cada $\mathcal{N} \in \mathcal{B}$ possui $\ddot{\alpha}$ tuplas do tipo $(i, \ell, \lambda, f, w, \sigma, \phi)$ que representam os nós candidatos oriundos da expansão do nível $\ell$ e da seleção da busca local. A linha 7 aplica a busca global em todos os nós em $\mathcal{B}$ e seleciona os $\ddot{\beta}$ melhores nós para continuarem a serem explorados. Após o laço principal, nas linhas 8-12, o método então retorna a melhor solução com $n$ tarefas sequenciadas em $\mathcal{N}$.

O Algoritmo 10 descreve a expansão dos $\eta$ primeiros níveis da árvore. Nestes níveis, para cada nó ativo, todas as possibilidades de expansão são considerados. Isso significa que todas as tarefas ainda não sequenciadas pelo nó serão avaliadas pela busca global. Inicialmente, as sequências parciais não possuem nenhuma tarefa. A primeira tarefa é escolhida baseada na regra SPT com soma dos tempos de processamento descrita em Sakuraba et al. (2009). O método LS da seção anterior também seleciona a primeira tarefa da sequência utilizando este critério. Contudo, o BS considera as $\min \{n, \kappa\}$ tarefas seguindo este critério. Para isso, a linha 2 calcula a somatória dos tempos de processamento para cada tarefa. A linha 3 cria um vetor de pares $(i, s p[i])$ onde $i$ é o índice de uma tarefa e $s p[i]$ é a somatória dos tempos de processamento da tarefa $i$. O vetor é então ordenado de acordo com os tempos de processamento do par e em caso de empates, de acordo com o menor índice da tarefa. O laço da linha 4 cria sequências com uma única tarefa, baseado na ordenação das tarefas pelos tempos de processamento, e completa esta sequência utilizando a rotina ComplETEWithFits. Essa rotina será descrita mais tarde neste texto, mas em palavras ela recebe uma sequência parcial qualquer, completa essa sequência utilizando os mesmos critérios do LS apresentado na seção anterior e então calcula os tempos de início de processamento para a sequência utilizando o Algoritmo 7. O laço então na linha 10 cria um conjunto $\mathcal{N}$ de tuplas $(1, f, w, \sigma, \phi)$ com sequências parciais de somente 


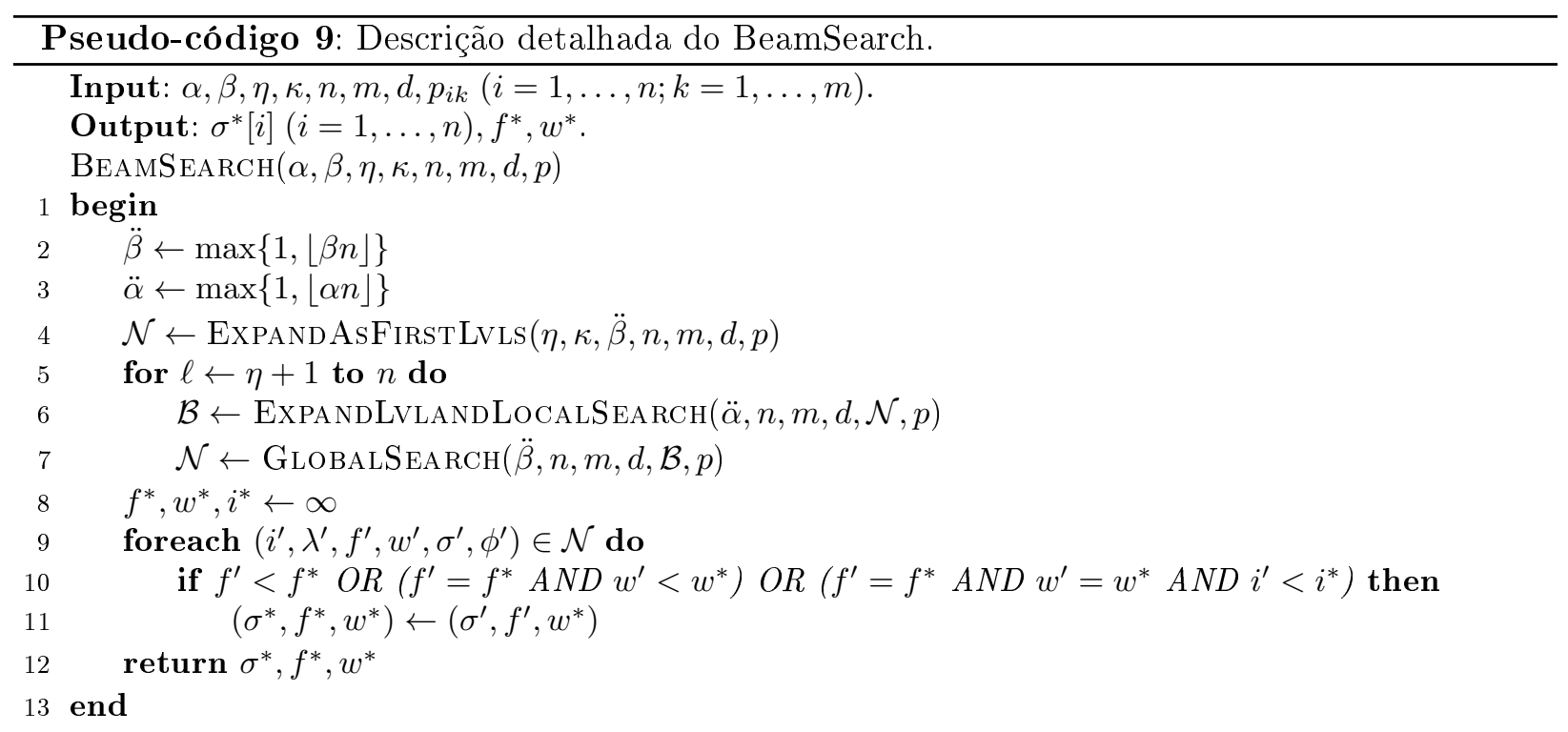

uma tarefa e valores $f$ e $w$ estimados pela rotina CompleteWithFits. O conjunto $\mathcal{N}$ é então ordenado na linha 11 de acordo com os valores estimados da soma dos adiantamentos e atrasos $f_{i}$. Em caso de empates, a soma dos atrasos $w_{i}$ é utilizada como desempate e como último critério de desempate, o índice da última tarefa sequenciada $j_{i}$. O laço das linhas $12-26$ itera $\eta-1$ vezes e a cada iteração, para cada nó em $\mathcal{N}$, gera todas as possibilidades de sequenciamento considerando todas as tarefas ainda não programadas (linhas 14-19), avalia as possibilidades (linha 20) e as armazena em $\mathcal{N}^{\prime}$. É importante perceber que os nós armazenados em $\mathcal{N}^{\prime}$ possuem uma tarefa a mais na sequência quando comparados com os nós de $\mathcal{N}$ neste ponto. Na linha 22 o conjunto $\mathcal{N}^{\prime}$ é ordenado de acordo com os valores de $f_{i}, w_{i}$ e $j_{i}$. Uma vez ordenado, os primeiros $\kappa$ nós de $\mathcal{N}^{\prime}$ são escolhidos para permanecerem na árvore e são adicionados em um novo $\mathcal{N}$. Após todas as iterações do laço da linha $12, \mathcal{N}$ terá até $\kappa$ nós com $\eta$ operações sequenciadas. O laço da linha 27 constrói então um conjunto $\hat{\mathcal{N}}$ com os $\beta$ melhores nós de $\mathcal{N}$ para ser o retorno do método.

o Algoritmo 11 descreve a rotina que completa uma solução parcial utilizando os critérios dos LS da seção anterior e em seguida calcula os inícios de processamento, a soma dos adiantamentos e atrasos e a soma dos tempos de espera entre as máquinas para a sequência obtida. A rotina recebe $\lambda, n, m, d, \sigma, \phi$ e $p$ como parâmetros. Nas linhas $2-10$ as $\lambda$ tarefas da sequência parcial $\sigma$ são programadas o mais cedo possível, quer dizer, instantes de tempo de término de processamento $c$ são calculados como os mais cedo possível para a sequência. As linhas 11 e 12 fazem uma cópia da sequência parcial $\sigma$ e do vetor $\phi$. O laço das linhas 13-21 então completa a sequência parcial escolhendo sempre a melhor tarefa possível com o auxílio da rotina OnERoundFiTs. A cada iteração do laço, a rotina OnERoundFits recebe a solução parcial atual $\sigma^{\prime}$ e devolve a melhor tarefa $i$ que satisfaça os mesmos critérios de seleção do LS da seção anterior tal que $\phi^{\prime}[i]=$ false. Nas linhas 15 e 16 a tarefa $i$ é adicionada à sequência $\sigma^{\prime}$ e marcada como já sequenciada. Instantes de término de processamento para a tarefa $i$ são então definidos no laço das linhas 19-21. Este laço itera $n-\lambda$ vezes e uma vez que em cada iteração adiciona uma tarefa à sequência de tamanho $\lambda$, no final $\sigma^{\prime}$ terá todas as tarefas adicionadas à sequência. Por fim, a linha 22 utiliza o Algoritmo 7 para calcular valores para a soma dos adiantamentos e dos atrasos $f$, para a soma dos tempos de espera $w$ e para os instantes de início de processamento st para a sequência $\sigma^{\prime}$.

O Algoritmo 12 descreve a rotina OneRoundFits. A rotina recebe um sequenciamento parcial $\sigma \operatorname{com} \lambda$ tarefas e o vetor $\phi$ que indica quais tarefas já foram sequenciadas. Além disso, a rotina também recebe os instantes de término de processamento $c$ de todas as tarefas que já estão em $\sigma$. A somatória dos tempos de processamento das tarefas é calculada na linha 2. Uma cópia $\sigma^{\prime}$ de tamanho $\lambda$ do sequenciamento parcial $\sigma$ é feita na linha 3. O laço das linhas 5-8 completa $\sigma^{\prime}$ a partir da posição $\lambda+1$ até a posição $n$ com as tarefas candidatas em ordem crescente, quer dizer, 


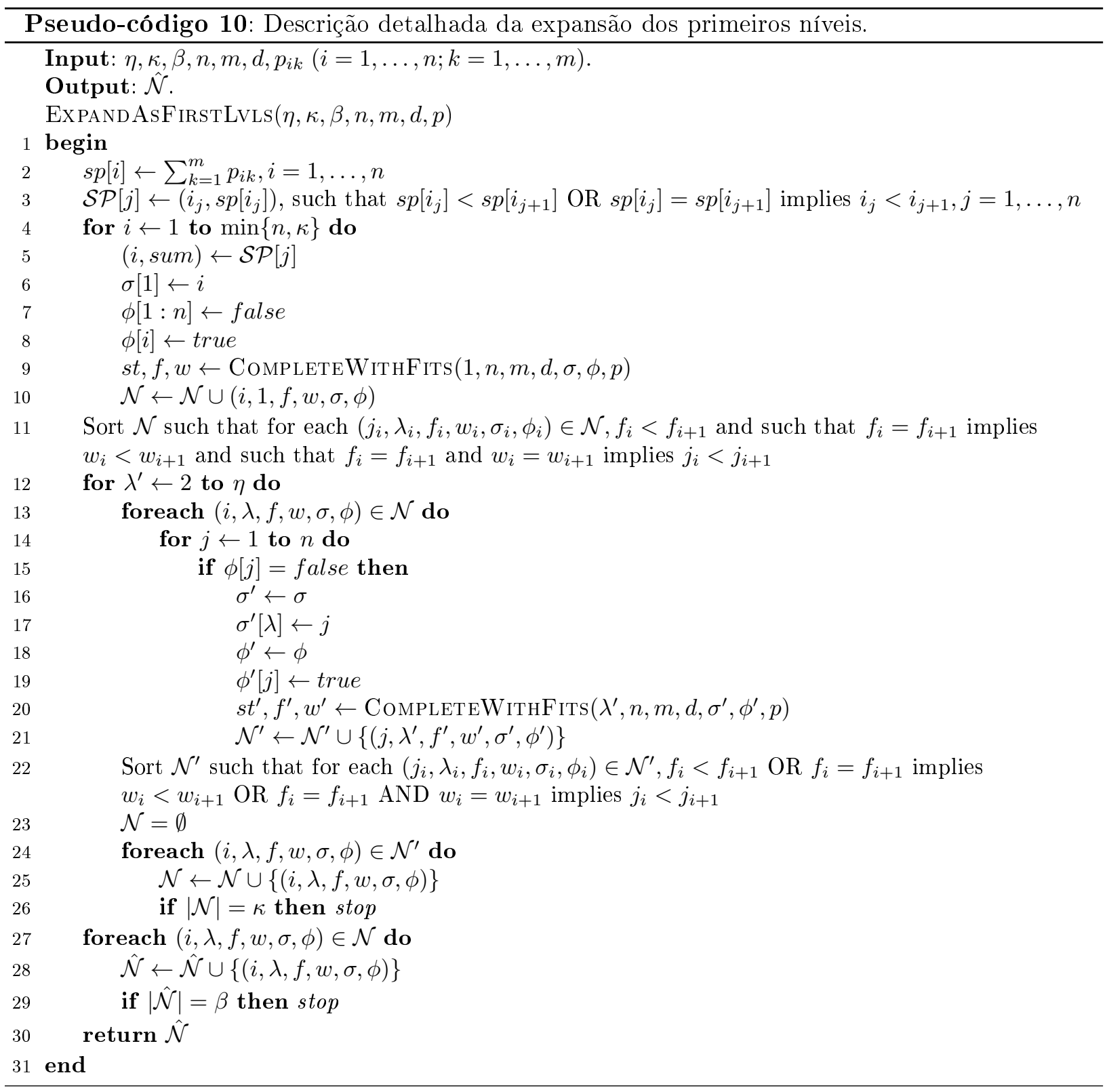

com as tarefas $i$ nas quais $\phi[i]=$ false. O laço das linhas 10-11 calcula as janelas da programação da sequência parcial $\sigma$. O laço das linhas 13-19, para cada tarefa $i$ que ainda não faz parte do sequenciamento parcial, calcula o encaixe $f$ it nas janelas (linha 15), calcula a estimativa do LS da seção anterior (linha 16) e verifica se a estimativa é a melhor encontrada até então (linha 17). Caso seja, fits* e $i^{*}$ são atualizados nas linhas 18 e 19. No final de suas iterações, fits* e $i^{*}$ serão o valor da melhor estimativa e a tarefa que originou esse valor respectivamente. A rotina retorna então, na linha 20, a tarefa $i^{*}$.

O Algoritmo 13 descreve a rotina ExPANDLvlandLocalSEARCH em detalhes A rotina recebe um conjunto $\mathcal{N}$ de nós do tipo $(i, \lambda, f, w, \sigma, \phi)$ do $\lambda$-ésimo nível da árvore do BS e para cada nó recebido, cria um conjunto $\mathcal{N}^{\prime}$ de até $\alpha$ nós do tipo $\left(j, \lambda+1, f^{\prime}, w^{\prime}, \sigma^{\prime}, \phi^{\prime}\right)$ que representam uma sequência parcial com $\lambda+1$ tarefas. As tarefas adicionadas são oriundas da avaliação da busca local. No algoritmo, a linha 2 inicializa o conjunto $\mathcal{B}$ como um conjunto vazio e a cada iteração do laço das linhas 3-16 adiciona um conjunto $\mathcal{N}^{\prime}$ à $\mathcal{B}$. Para isso, para cada nó $(i, \lambda, f, w, \sigma, \phi)$ em $\mathcal{N}$, a rotina AlloneRoundFits armazena em $\mathcal{O}$ pares do tipo $(i, v)$ na linha 4 onde $i$ é uma tarefa ainda não sequenciada, quer dizer, $\phi[i]=$ false e $v$ é o valor do critério de seleção do LS da seção anterior. Na linha 5, o conjunto $\mathcal{O}$ é ordenado de acordo com os valores $v$ de cada par e em casos de empates, de acordo com os índices $i$. O laço das linhas $7-15$ então, para cada par $\left(i^{\prime}, v^{\prime}\right)$ do conjunto $\mathcal{O}$, faz 

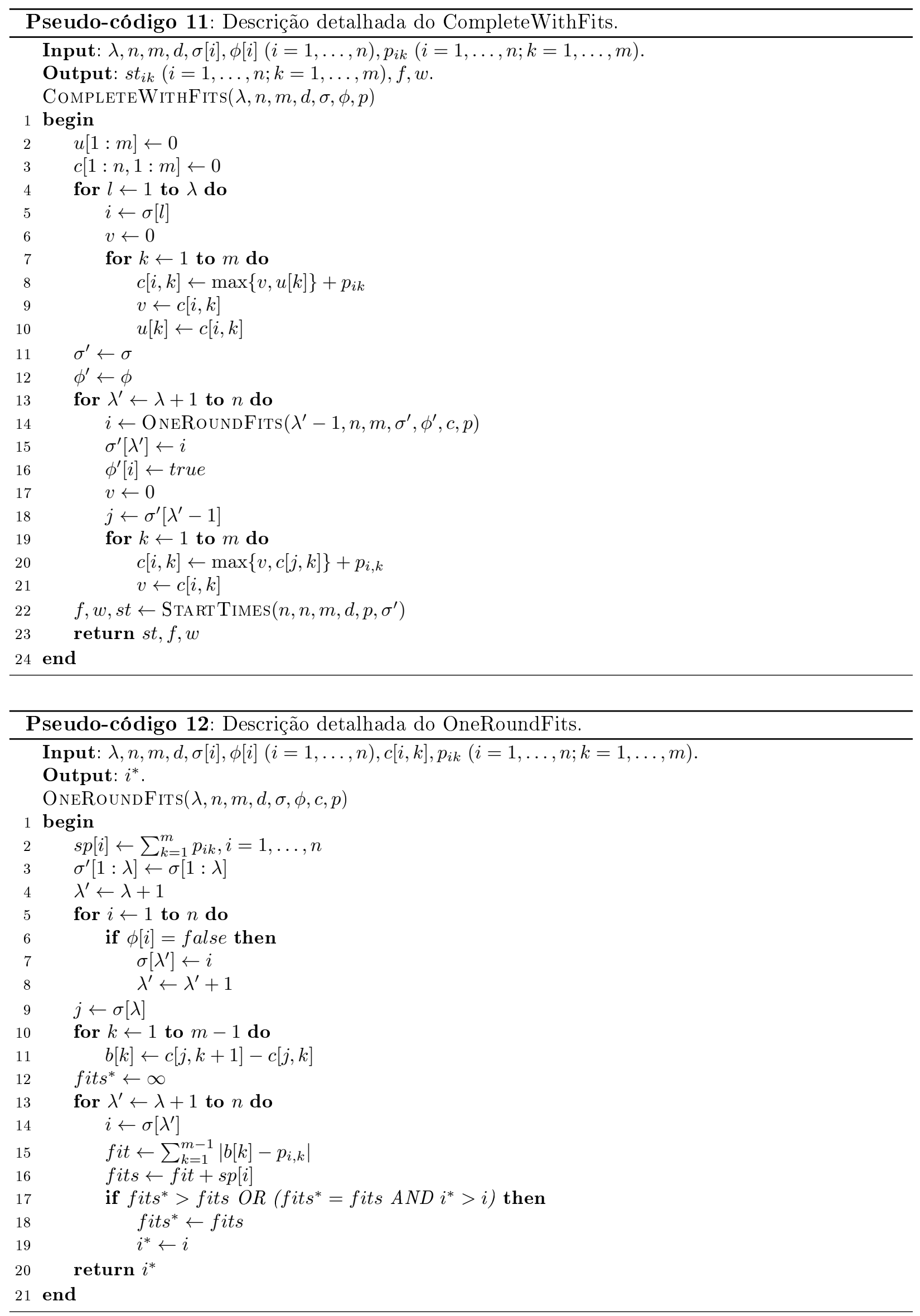
uma cópia $\sigma^{\prime}$ do sequenciamento parcial $\sigma$ (linha 9), adiciona $i^{\prime}$ ao sequenciamento $\sigma^{\prime}$ (linha 10), marca $i^{\prime}$ como já sequenciada (linhas 11-12), calcula os valores da soma dos adiantamentos e atrasos $f^{\prime}$, dos tempos de espera $w^{\prime}$ e dos instantes de início de processamento para o sequenciamento $\sigma^{\prime}$ com o Algoritmo 7 (linha 13) e então adiciona o nó expandido $\left(i^{\prime}, \lambda^{\prime}, f^{\prime}, w^{\prime}, \sigma^{\prime}, \phi^{\prime}\right)$ no conjunto $\mathcal{N}^{\prime}$ (linha 14). Este laço é repetido $|\mathcal{O}|$ vezes ou até que $\mathcal{N}^{\prime}$ tenha $\alpha$ elementos. Por último, o conjunto de nós expandidos e avaliados pela busca local $\mathcal{N}^{\prime}$ é adicionado ao conjunto $\mathcal{B}$ na linha 16 . O método retorna o conjunto $\mathcal{B}$ (linha 17 ).

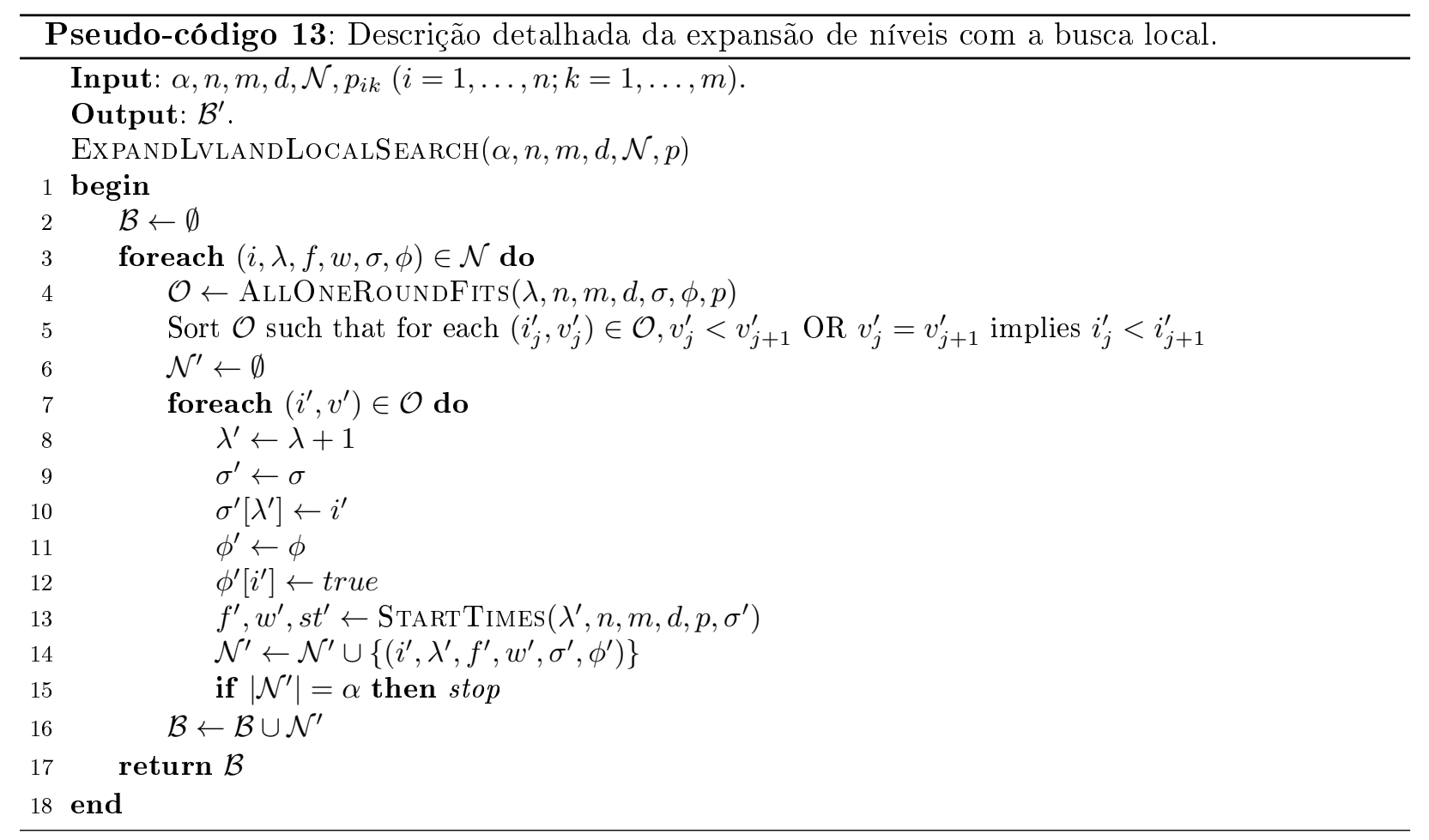

O Algoritmo 14 descreve o pseudocódigo da rotina AllOneRoundFits em detalhes. O algoritmo recebe uma sequência parcial $\sigma$ com $\lambda$ tarefas já sequenciadas e devolve um conjunto $\mathcal{O}$ de pares $(i, f i t s)$ em que $i$ é uma tarefa ainda não sequenciada, quer dizer, $\phi[i]=$ false e fits é o valor da estimativa do LS da seção anterior para a tarefa $i$ considerando o sequenciamento parcial $\sigma$. No algoritmo, as linhas 2-10 calculam os instantes de término de processamento da sequência parcial $\sigma$ como o mais cedo possível. A linha 11 armazena em $j$ a última tarefa da sequência parcial. O laço das linhas 12-13 calcula as janelas do sequenciamento parcial. A somatória dos tempos de processamento para cada tarefa é calculada na linha 14. O laço das linhas $15-19$, para cada tarefa candidata $i$, calcula a estimativa fits (linhas $17-18$ ) e adiciona em $\mathcal{O}$ o par ( $i$, fits) correspondente (linha 19). Por fim, o conjunto $\mathcal{O}$ é retornado (linha 20).

O Algoritmo 15 descreve o pseudocódigo da rotina GlobalSearch em detalhes. A rotina recebe um conjunto $\mathcal{B}$ de conjuntos $\mathcal{N}$ de nós do tipo $\left(i^{\prime}, \lambda^{\prime}, f^{\prime}, w^{\prime}, \sigma^{\prime}, \phi^{\prime}\right)$, completa todos os nós utilizando a rotina CompleteWithFits (Algoritmo 11), avalia o sequenciamento completo com o Algoritmo 7, armazena em $\mathcal{N}$ os $\beta$ melhores sequenciamentos parciais que resultaram os melhores sequenciamentos completos de acordo com a avaliação e retorna $\mathcal{N}$. Para isso, o laço das linhas 3-6 completa cada nó em $\mathcal{B}$ (linha 5), avalia o sequenciamento completo (linha 5) e armazena a sequência parcial $\sigma^{\prime}$ e os valores da avaliação $f$ e $w$ no conjunto $\hat{\mathcal{N}}$ (linha 6 ). Na linha 7 , o conjunto $\hat{\mathcal{N}}$ é ordenado de acordo com os valores armazenados. Por último, o laço das linhas 9-11 adiciona em $\mathcal{N}$ os $\beta$ melhores nós de $\hat{\mathcal{N}}$ (linhas 10 e 11). 

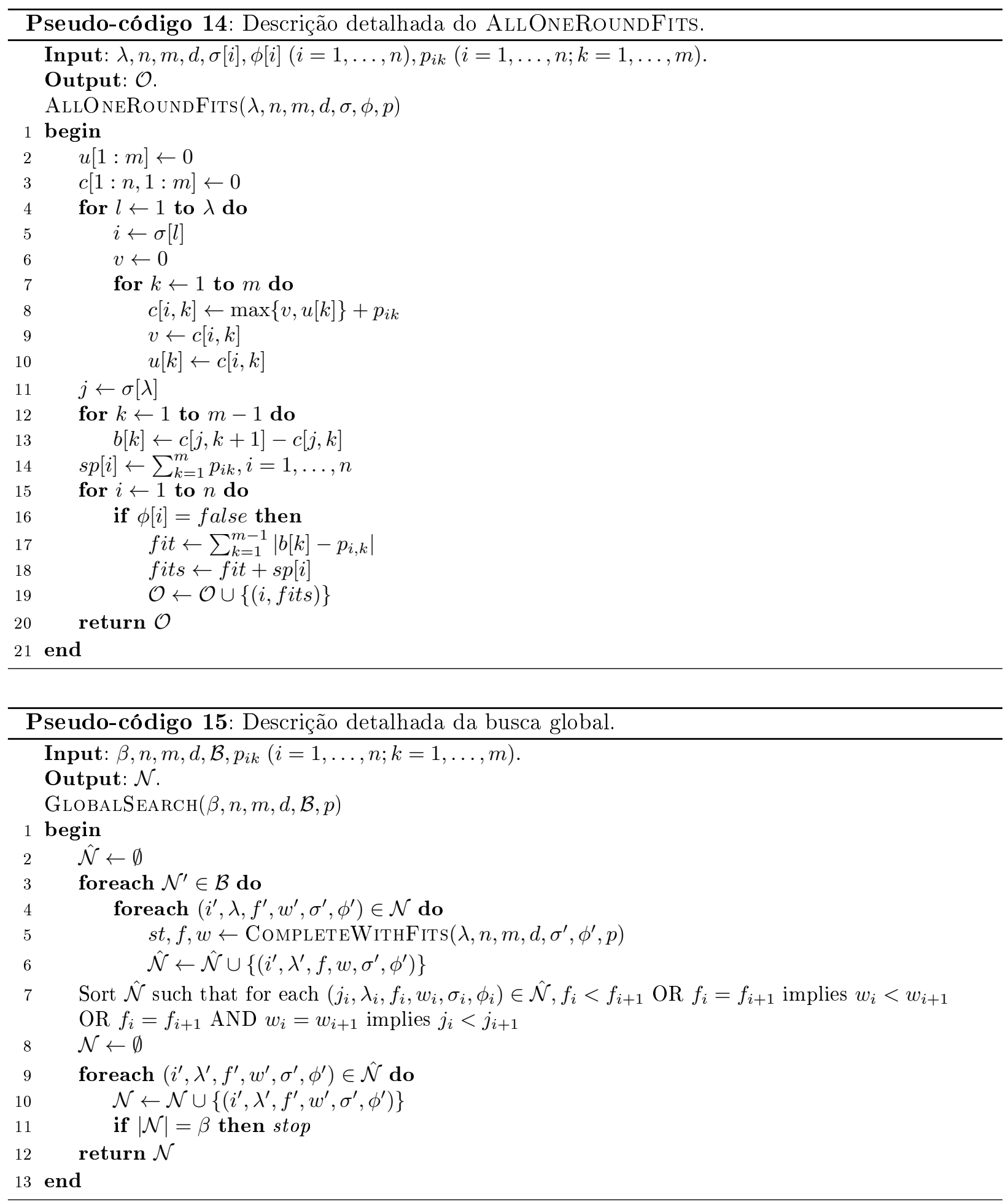

\subsection{Experimentos Numéricos}

Todos os algoritmos apresentados neste capítulo, foram implementados em $\mathrm{C} / \mathrm{C}++$. A compilação dos códigos foi feita utilizando o compilador g++ do GCC, versão 6.3.0 Debian 6.3.0-18, utilizando a opção de otimização "-O3". Todos os testes foram feitos utilizando um computador Intel(R) Xeon(R) E5645 2.40GHz com 216 GB de memória RAM e executados no sistema operacional Debian 9 x86_64.

Nos testes computacionais, o conjunto de 1200 instâncias e as 2 datas restritas de entrega propostos em Chandra et al. (2009) foram utilizados. Essas instâncias foram geradas utilizando a 
seguinte combinação de parâmetros: $n \in\{5,10,20,50,80,100\}, m \in\{5,10,15,20\}$ e $p_{i k} \in[1,99]$, $i=1, \ldots, n$ e $k=1, \ldots, m$. O conjunto possui 50 instâncias para cada combinação de $n$ e $m$.

Inicialmente, o algoritmo LS proposto neste capítulo foi comparado com dois outros algoritmos da mesma categoria da literatura. O primeiro, aqui denominado HS1, consiste no algoritmo construtivo baseado em regras de despacho que foi utilizado para construir uma solução inicial para a busca tabu de Chandra et al. (2009). O segundo, o SPT que considera a somatória dos tempos de processamento de Sakuraba et al. (2009) e que também foi utilizado para construir a solução inicial da busca tabu dos autores. Para avaliar o desempenho dos três algoritmos em questão, as regras foram executadas no conjunto de instâncias para as duas datas de entrega e a programação das sequências obtidas foi calculada pelo Algoritmo 7. A partir dos valores de soma dos adiantamentos e dos atrasos $(E / T)$ e das somas dos tempos de espera $(W)$ obtidos, o IDR (índice de desvio relativo)

$$
I D R=\frac{V-\min }{\max -\min }
$$

foi calculado, sendo $V$ o valor em questão, min o menor entre os valores e max o maior entre os valores. As Tabelas 3.6 e 3.7 apresentam os resultados para as duas datas de entrega. Na tabela, cada entrada representa uma média dos valores obtidos em 50 instâncias nas quais $n$ é a quantidade de tarefas e $m$ a quantidade de máquinas. $W$ é a média do $I D R$ da soma dos tempos de espera somente nos casos em que duas ou mais soluções encontraram o menor valor de $E / T$, caracterizando um empate. \#Empates é a quantidade de vezes que dois ou mais métodos encontraram o menor valor de $E / T$. De acordo com as tabelas, houve somente 47 e 43 empates de $E / T$ em todas as 1200 instâncias consideradas para $d^{\prime}$ e $d^{\prime \prime}$, respectivamente. Todavia, em nenhum caso as soluções tinham valores de soma de tempos de espera entre as máquinas $W$ diferentes. No geral, o algoritmo LS proposto nesta tese para o FSP-WT consegue resultados melhores do que os métodos da literatura em 22 das 24 configurações de instâncias diferentes para $d^{\prime}$ e em 21 das 24 para $d^{\prime \prime}$. É notável também que a medida que a complexidade das instâncias aumenta, o LS consegue desempenho cada vez melhor e que nas instâncias com $n \geq 50$, a média dos $I D R$ do método é 0 ou praticamente 0 em todos os casos. Em linhas gerais, o LS foi o melhor método, com média geral dos $I D R$ para $E / T$ de 0.12 e 0.18 enquanto o SPT foi o segundo melhor método com médias dos $I D R$ para $E / T$ de 0.38 e 0.46 , respectivamente. O LS, o SPT e o HS1 levam em média 0.005 segundos, 0.001 segundos e 0.02 segundos de tempo computacional respectivamente para solucionar uma instância do conjunto considerado.

Em relação ao BS deste capítulo, inicialmente vários testes preliminares foram feitos para a calibragem dos parâmetros $\kappa, \alpha$ e $\beta$. Nestes testes iniciais, somente 240 instâncias foram consideradas, sendo 10 instâncias para cada combinação de $n$ e $m$. Os valores analisados para $\kappa$ foram de $60,80,100$ e 120 . Os valores testados para $\alpha$ e $\beta$ foram de $0.25,0.50,0.75$ e 1.00. Em relação ao parâmetro $\kappa$, os diferentes valores avaliados praticamente não impactaram nos resultados. A diferença de tempo de processamento em CPU também foi muito pequena e por isso, os testes para este parâmetro serão omitidos e fica definido que $\kappa=120$ para os demais resultados para o BS neste capítulo.

As Tabelas 3.8 e 3.9 apresentam os resultados obtidos para os diferentes valores de $\alpha$ e $\beta$ e data de entrega $d^{\prime}$ e $d^{\prime \prime}$, respectivamente. De acordo com as tabelas, a melhor configuração de parâmetros é $\beta=1.00$ e $\alpha=0.50$, com médias de $E / T$ iguais a 0.13 e 0.12 , respectivamente. Apesar dos 146 empates para $d^{\prime}$ e dos 129 empates para $d^{\prime \prime}$, não houve variação significativa para $W$. A Figura 3.12 ilustra graficamente a média dos tempos computacionais em segundos para as diferentes configurações analisadas. De acordo com os gráficos, como esperado, é fácil ver a relação de aumento de tempo com o aumento dos parâmetros $\alpha$ e $\beta$. A configuração $\alpha=0.25$ e $\beta=0.25$ é a configuração com menor tempo computacional. A configuração com maior tempo computacional é $\alpha=1.00$ e $\beta=1.00$. A configuração $\alpha=0.50$ e $\beta=1.00$ gastou aproximadamente 478 segundos por instância.

Uma vez definidos os parâmetros do método BS $(\kappa=120, \alpha=0.50$ e $\beta=1.00)$, uma comparação 


\begin{tabular}{cc|cc|cc|cc|c}
\hline \hline \multicolumn{10}{c|}{$d^{\prime}$} \\
\hline \hline \multicolumn{1}{c}{ Instância } & \multicolumn{2}{|c|}{ HS1 } & \multicolumn{2}{c}{ SPT } & \multicolumn{2}{c}{ LS } & \# Empates \\
$n$ & $m$ & $E / T$ & $W$ & $E / T$ & $W$ & $E / T$ & $W$ & \\
\hline \hline 5 & 5 & 0.56 & 0.00 & 0.50 & 0.00 & 0.29 & 0.00 & 15 \\
5 & 10 & 0.64 & 0.00 & 0.32 & 0.00 & 0.42 & 0.00 & 11 \\
5 & 15 & 0.54 & 0.00 & 0.47 & 0.00 & 0.39 & 0.00 & 8 \\
5 & 20 & 0.45 & 0.00 & 0.48 & 0.00 & 0.52 & 0.00 & 13 \\
10 & 5 & 0.91 & 0.00 & 0.29 & 0.00 & 0.11 & 0.00 & 0 \\
10 & 10 & 0.64 & 0.00 & 0.51 & 0.00 & 0.21 & 0.00 & 0 \\
10 & 15 & 0.67 & 0.00 & 0.43 & 0.00 & 0.30 & 0.00 & 0 \\
10 & 20 & 0.59 & 0.00 & 0.49 & 0.00 & 0.32 & 0.00 & 0 \\
20 & 5 & 0.96 & 0.00 & 0.30 & 0.00 & 0.02 & 0.00 & 0 \\
20 & 10 & 0.93 & 0.00 & 0.36 & 0.00 & 0.11 & 0.00 & 0 \\
20 & 15 & 0.89 & 0.00 & 0.45 & 0.00 & 0.04 & 0.00 & 0 \\
20 & 20 & 0.84 & 0.00 & 0.48 & 0.00 & 0.10 & 0.00 & 0 \\
50 & 5 & 1.00 & 0.00 & 0.21 & 0.00 & 0.00 & 0.00 & 0 \\
50 & 10 & 1.00 & 0.00 & 0.40 & 0.00 & 0.01 & 0.00 & 0 \\
50 & 15 & 0.99 & 0.00 & 0.42 & 0.00 & 0.00 & 0.00 & 0 \\
50 & 20 & 0.95 & 0.00 & 0.46 & 0.00 & 0.02 & 0.00 & 0 \\
80 & 5 & 1.00 & 0.00 & 0.16 & 0.00 & 0.00 & 0.00 & 0 \\
80 & 10 & 1.00 & 0.00 & 0.32 & 0.00 & 0.00 & 0.00 & 0 \\
80 & 15 & 1.00 & 0.00 & 0.41 & 0.00 & 0.00 & 0.00 & 0 \\
80 & 20 & 1.00 & 0.00 & 0.45 & 0.00 & 0.00 & 0.00 & 0 \\
100 & 5 & 1.00 & 0.00 & 0.12 & 0.00 & 0.00 & 0.00 & 0 \\
100 & 10 & 1.00 & 0.00 & 0.28 & 0.00 & 0.00 & 0.00 & 0 \\
100 & 15 & 1.00 & 0.00 & 0.42 & 0.00 & 0.00 & 0.00 & 0 \\
100 & 20 & 1.00 & 0.00 & 0.40 & 0.00 & 0.00 & 0.00 & 0 \\
\hline \hline \multicolumn{2}{l}{ Média } & 0.86 & 0.00 & 0.38 & 0.00 & 0.12 & 0.00 & - \\
& & & & & & & & \\
\hline
\end{tabular}

Tabela 3.6: Resultados computacionais para o LS proposto neste trabalho considerando a data de entrega $d^{\prime}$. Na tabela, cada entrada de E/T é a média dos IDR da soma dos adiantamentos e dos atrasos para 50 instâncias com $n$ tarefas e m máquinas. \# Empates é a quantidade de vezes que dois ou mais métodos encontraram o melhor valor de $E / T$. W é o IDR da soma dos tempos de espera nos casos em que houveram empates para $E / T$.

dos resultados obtidos pelo LS e pelo BS em relação aos resultados obtidos pela busca tabu de Chandra et al. (2009) no conjunto completo de 1200 instâncias foi feita. Para isso, a distância relativa (gap) da soma dos adiantamentos e atrasos obtidas pelos métodos em relação à soma obtida pela busca tabu de Chandra et al. (2009) foi calculada, onde

$$
g a p=100 \times \frac{V-C V}{C V},
$$

sendo $V$ o obtido pelo método e $C V$ o valor reportado por Chandra et al. (2009). As Tabelas 3.10 e 3.11 apresentam os resultados. Nas tabelas, $\overline{\text { gap }}$ é a média do gap em 50 instâncias com $n$ tarefas e $m$ máquinas. \# Vitórias é a quantidade de vezes em que o método em questão conseguiu gap menor ou igual a zero, quer dizer, a quantidade de vezes em que o método conseguiu atingir ou superar os resultados de Chandra et al. (2009). \# Empates é a quantidade de vezes em que os métodos encontraram o mesmo valor de soma de adiantamentos e atrasos entre si. De acordo com as tabelas, os métodos só obtiveram os mesmos resultados de gap em 16 dos 2400 casos. O LS fica a aproximadamente $27.29 \%$ e $28.93 \%$ de distância relativa em relação a busca tabu de Chandra et al. (2009). O BS supera a busca tabu de Chandra et al. (2009) em $-3.73 \%$ e $-1.97 \%$. Os resultados 


\begin{tabular}{cc|cc|cc|cc|c}
\hline \hline \multicolumn{10}{c|}{$d^{\prime \prime}$} \\
\hline \hline \multicolumn{2}{c|}{ Instância } & \multicolumn{2}{|c|}{ HS1 } & \multicolumn{2}{c}{ SPT } & \multicolumn{2}{c}{ LS } & \multirow{2}{*}{ Empates } \\
\hline \hline & $m$ & $E / T$ & $W$ & $E / T$ & $W$ & $E / T$ & $W$ & \\
\hline 5 & 5 & 0.49 & 0.00 & 0.54 & 0.00 & 0.36 & 0.00 & 15 \\
5 & 10 & 0.54 & 0.00 & 0.40 & 0.00 & 0.49 & 0.00 & 9 \\
5 & 15 & 0.44 & 0.00 & 0.54 & 0.00 & 0.53 & 0.00 & 6 \\
5 & 20 & 0.47 & 0.00 & 0.48 & 0.00 & 0.51 & 0.00 & 12 \\
10 & 5 & 0.75 & 0.00 & 0.41 & 0.00 & 0.16 & 0.00 & 0 \\
10 & 10 & 0.56 & 0.00 & 0.59 & 0.00 & 0.34 & 0.00 & 0 \\
10 & 15 & 0.62 & 0.00 & 0.48 & 0.00 & 0.33 & 0.00 & 1 \\
10 & 20 & 0.61 & 0.00 & 0.48 & 0.00 & 0.32 & 0.00 & 0 \\
20 & 5 & 0.77 & 0.00 & 0.43 & 0.00 & 0.13 & 0.00 & 0 \\
20 & 10 & 0.75 & 0.00 & 0.52 & 0.00 & 0.16 & 0.00 & 0 \\
20 & 15 & 0.69 & 0.00 & 0.52 & 0.00 & 0.20 & 0.00 & 0 \\
20 & 20 & 0.69 & 0.00 & 0.51 & 0.00 & 0.18 & 0.00 & 0 \\
50 & 5 & 0.95 & 0.00 & 0.29 & 0.00 & 0.04 & 0.00 & 0 \\
50 & 10 & 0.86 & 0.00 & 0.50 & 0.00 & 0.05 & 0.00 & 0 \\
50 & 15 & 0.87 & 0.00 & 0.46 & 0.00 & 0.08 & 0.00 & 0 \\
50 & 20 & 0.85 & 0.00 & 0.55 & 0.00 & 0.07 & 0.00 & 0 \\
80 & 5 & 0.93 & 0.00 & 0.27 & 0.00 & 0.03 & 0.00 & 0 \\
80 & 10 & 0.84 & 0.00 & 0.46 & 0.00 & 0.05 & 0.00 & 0 \\
80 & 15 & 0.86 & 0.00 & 0.48 & 0.00 & 0.08 & 0.00 & 0 \\
80 & 20 & 0.91 & 0.00 & 0.51 & 0.00 & 0.07 & 0.00 & 0 \\
100 & 5 & 0.93 & 0.00 & 0.20 & 0.00 & 0.03 & 0.00 & 0 \\
100 & 10 & 0.90 & 0.00 & 0.41 & 0.00 & 0.04 & 0.00 & 0 \\
100 & 15 & 0.86 & 0.00 & 0.49 & 0.00 & 0.09 & 0.00 & 0 \\
100 & 20 & 0.85 & 0.00 & 0.51 & 0.00 & 0.09 & 0.00 & 0 \\
\hline \hline \multicolumn{2}{|c|}{ Média } & 0.75 & 0.00 & 0.46 & 0.00 & 0.18 & 0.00 & - \\
& & & & & & & & \\
\hline
\end{tabular}

Tabela 3.7: Resultados computacionais para o LS proposto neste trabalho considerando a data de entrega $d^{\prime \prime}$. Na tabela, cada entrada de E/T é a média dos IDR da soma dos adiantamentos e dos atrasos para 50 instâncias com $n$ tarefas e $m$ máquinas. \# Empates é a quantidade de vezes que dois ou mais métodos encontraram o melhor valor de E/T.W é o IDR da soma dos tempos de espera nos casos em que houveram empates para $E / T$.

\begin{tabular}{c|cc|cc|cc|cc}
\hline \hline \multicolumn{10}{|c}{$d^{\prime}$} \\
\hline \hline & \multicolumn{2}{|c|}{$\alpha=0.25$} & \multicolumn{2}{c|}{$\alpha=0.50$} & \multicolumn{2}{c|}{$\alpha=0.75$} & \multicolumn{2}{c}{$\alpha=1.00$} \\
& $E / T$ & $W$ & $E / T$ & $W$ & $E / T$ & $W$ & $E / T$ & $W$ \\
\hline \hline$\beta=0.25$ & 0.58 & 0.00 & 0.51 & 0.00 & 0.48 & 0.00 & 0.49 & 0.00 \\
$\beta=0.50$ & 0.39 & 0.00 & 0.33 & 0.00 & 0.33 & 0.00 & 0.33 & 0.00 \\
$\beta=0.75$ & 0.29 & 0.00 & 0.23 & 0.00 & 0.22 & 0.00 & 0.23 & 0.00 \\
$\beta=1.00$ & 0.21 & 0.00 & 0.13 & 0.00 & 0.15 & 0.00 & 0.14 & 0.00 \\
\hline \hline
\end{tabular}

Tabela 3.8: Resultados computacionais preliminares para o BS com diferentes valores para os parâmetros $\alpha$ e $\beta$ utilizando um conjunto reduzido de instâncias e data de entrega d'. Na tabela, E/T é a média dos IDR da soma dos adiantamentos e dos atrasos encontrados pela BS em relação às demais configurações do método. W é o IDR da soma dos tempos de espera nos casos em que uma ou mais configurações encontraram o mesmo valor de $E / T$.

em relação ao \# Vitórias são notáveis. Enquanto o LS consegue atingir ou superar os resultados da busca tabu em 20 dos 2400 casos, o BS conseguiu atingir ou superar os resultados em 2065 dos 


\begin{tabular}{c|cc|cc|cc|cc}
\hline \hline \multicolumn{10}{|c}{$d^{\prime \prime}$} \\
\hline \hline & \multicolumn{2}{|c|}{$\alpha=0.25$} & \multicolumn{2}{c|}{$\alpha=0.50$} & \multicolumn{2}{c}{$\alpha=0.75$} & \multicolumn{2}{c}{$\alpha=1.00$} \\
& $E / T$ & $W$ & $E / T$ & $W$ & $E / T$ & $W$ & $E / T$ & $W$ \\
\hline \hline$\beta=0.25$ & 0.60 & 0.00 & 0.49 & 0.00 & 0.50 & 0.00 & 0.50 & 0.00 \\
$\beta=0.50$ & 0.37 & 0.00 & 0.33 & 0.00 & 0.34 & 0.00 & 0.35 & 0.00 \\
$\beta=0.75$ & 0.27 & 0.00 & 0.20 & 0.00 & 0.25 & 0.00 & 0.24 & 0.00 \\
$\beta=1.00$ & 0.21 & 0.00 & 0.12 & 0.00 & 0.16 & 0.00 & 0.16 & 0.00 \\
\hline \hline
\end{tabular}

Tabela 3.9: Resultados computacionais preliminares para o BS com diferentes valores para os parâmetros $\alpha$ e $\beta$ utilizando um conjunto reduzido de instâncias e data de entrega d". Na tabela, E/T é a média dos $I D R$ da soma dos adiantamentos e dos atrasos encontrados pela $B S$ em relação às demais configurações do método. W é o IDR da soma dos tempos de espera nos casos em que uma ou mais configurações encontraram o mesmo valor de $E / T$.

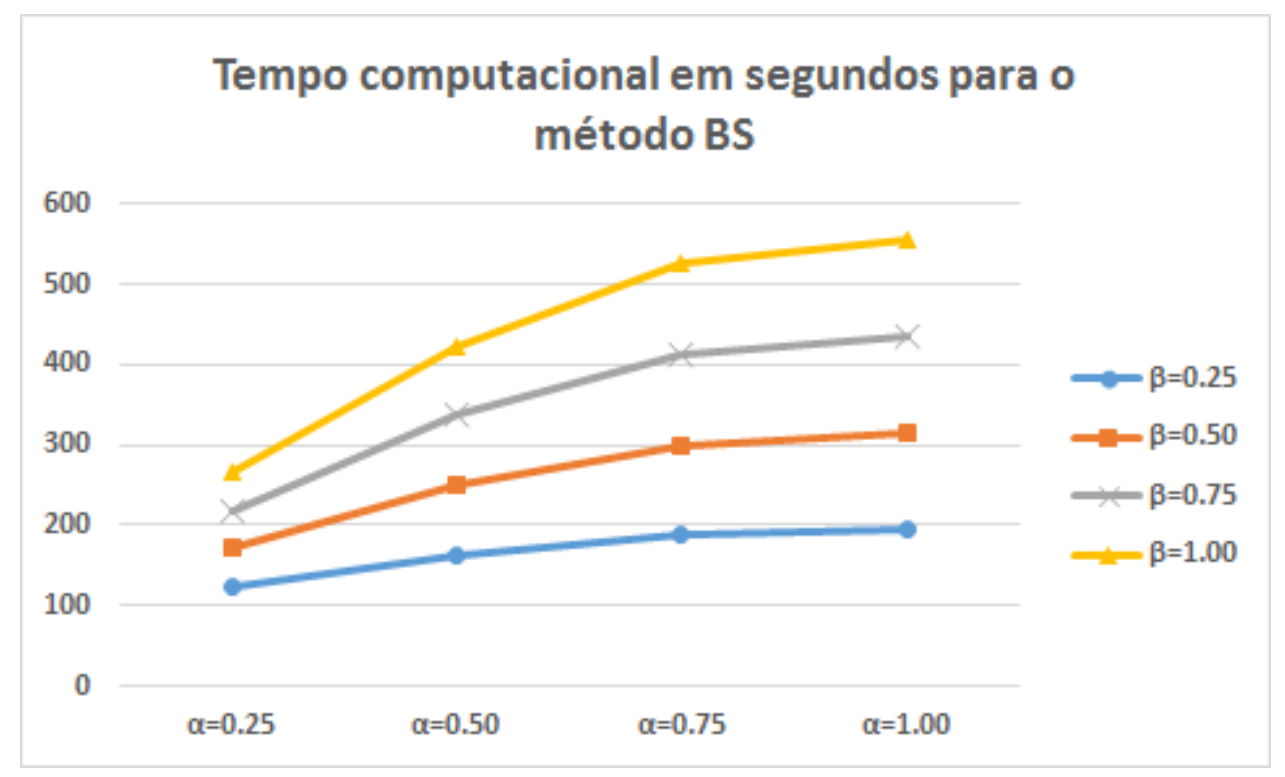

Figura 3.12: Tempo computacional médio em segundos gasto pelo BS com diferentes configurações de $\alpha$ e $\beta$ para solucionar uma instância.

2400.

Além da comparação feita com os resultados reportados por Chandra et al. (2009), os métodos também foram comparados em relação a um solver exato executado sobre o modelo matemático descrito na Seção 3.1. Para isso, devido a complexidade do problema, somente as instâncias de pequeno porte do conjunto de instâncias total foram utilizadas. Estas instâncias possuem configurações de $(n, m)$ iguais a $(5,5),(5,10),(5,15),(5,20)$ e $(10,5)$. As Tabelas 3.12 e 3.13 apresentam os resultados para $d^{\prime}$ e $d^{\prime \prime}$ respectivamente. De acordo com as tabelas, o método LS conseguiu atingir resultado ótimo para soma dos adiantamentos e atrasos em somente 16 dos 500 casos e ficou com média geral de gap em torno de $40 \%$ enquanto o método BS conseguiu ótimos em 455 dos 500 casos e média geral em torno de $0.12 \%$. Os métodos empataram somente em 16 casos. Estes casos de empate são exatamente os mesmos casos onde o LS conseguiu atingir resultados ótimos. Nestes casos, a soma dos tempos de espera entre as máquinas foram exatamente as mesmas e por isso $W$ possui o mesmo valor nas tabelas para ambos os métodos. 


\begin{tabular}{|c|c|c|c|c|c|c|c|c|}
\hline \multicolumn{9}{|c|}{$\overline{d^{\prime}}$} \\
\hline \multicolumn{2}{|c|}{ Instância } & \multicolumn{3}{|c|}{ 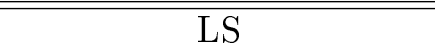 } & \multicolumn{3}{|c|}{$\overline{\mathrm{BSS}}$} & \multirow{2}{*}{ \# Empates } \\
\hline$n$ & $m$ & $\overline{g a p}$ & $W$ & \# Vitórias & $\overline{g a p}$ & $W$ & \# Vitórias & \\
\hline 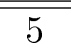 & 5 & 27.72 & 0.00 & 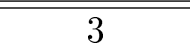 & -0.86 & (0.00 & 50 & 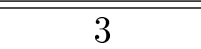 \\
\hline 5 & 10 & 40.73 & 0.00 & 3 & -1.02 & 0.00 & 50 & 2 \\
\hline 5 & 15 & 38.27 & 0.00 & 3 & -1.02 & 0.00 & 50 & 3 \\
\hline 5 & 20 & 58.03 & 0.00 & 0 & -0.93 & 0.00 & 50 & 0 \\
\hline 10 & 5 & 27.04 & 0.00 & 0 & -0.72 & 0.00 & 42 & 0 \\
\hline 10 & 10 & 34.70 & 0.00 & 0 & -1.45 & 0.00 & 42 & 0 \\
\hline 10 & 15 & 43.64 & 0.00 & 0 & -1.41 & 0.00 & 41 & 0 \\
\hline 10 & 20 & 43.30 & 0.00 & 0 & -1.16 & 0.00 & 37 & 0 \\
\hline 20 & 5 & 23.75 & 0.00 & 0 & -2.18 & 0.00 & 36 & 0 \\
\hline 20 & 10 & 31.51 & 0.00 & 0 & -2.82 & 0.00 & 45 & 0 \\
\hline 20 & 15 & 30.45 & 0.00 & 0 & -3.54 & 0.00 & 43 & 0 \\
\hline 20 & 20 & 32.57 & 0.00 & 0 & -4.33 & 0.00 & 44 & 0 \\
\hline 50 & 5 & 19.18 & 0.00 & 0 & -2.53 & 0.00 & 48 & 0 \\
\hline 50 & 10 & 22.09 & 0.00 & 0 & -5.35 & 0.00 & 50 & 0 \\
\hline 50 & 15 & 22.61 & 0.00 & 0 & -5.85 & 0.00 & 50 & 0 \\
\hline 50 & 20 & 24.24 & 0.00 & 0 & -5.71 & 0.00 & 49 & 0 \\
\hline 80 & 5 & 17.64 & 0.00 & 0 & -2.92 & 0.00 & 48 & 0 \\
\hline 80 & 10 & 17.24 & 0.00 & 0 & -6.15 & 0.00 & 50 & 0 \\
\hline 80 & 15 & 18.41 & 0.00 & 0 & -7.01 & 0.00 & 50 & 0 \\
\hline 80 & 20 & 18.41 & 0.00 & 0 & -7.35 & 0.00 & 50 & 0 \\
\hline 100 & 5 & 15.55 & 0.00 & 0 & -3.59 & 0.00 & 49 & 0 \\
\hline 100 & 10 & 16.44 & 0.00 & 0 & -6.39 & 0.00 & 50 & 0 \\
\hline 100 & 15 & 15.16 & 0.00 & 0 & -7.31 & 0.00 & 50 & 0 \\
\hline 100 & 20 & 16.33 & 0.00 & 0 & -7.87 & 0.00 & 50 & 0 \\
\hline$\overline{\mathrm{M}}$ & & 27.29 & 0.00 & 0.38 & -3.73 & 0.00 & 446.83 & - \\
\hline
\end{tabular}

Tabela 3.10: Resultados computacionais dos métodos LS e BS propostos neste capítulo considerando as 1200 instâncias e a data de entrega $d^{\prime}$. Na tabela, cada entrada de $\overline{g a p}$ é a média dos gap relativos da soma dos adiantamentos e dos atrasos encontrados pelos métodos em relação aos resultados reportados por Chandra et al. (2009) em 50 instâncias com n tarefas e m máquinas. \# Empates é a quantidade de vezes que os dois métodos encontraram o mesmo valor de gap. W é o IDR da soma dos tempos de espera nesses casos. \# Vitórias é a quantidade de vezes em que o método conseguiu atingir ou superar os resultados reportados por Chandra et al. (2009).

\subsection{Conclusão}

Este capítulo aborda uma extensão do FSP com sequência de permutação na qual os tempos de espera das tarefas entre as máquinas são considerados. Para o desenvolvimento dos métodos utilizados como abordagem, a mesma metodologia do Capítulo 2 foi utilizada. Nesta linha, inicialmente uma heurística construtiva do tipo list scheduling, denominada FITs, foi desenvolvida. A cada iteração, a heurística escolhe, entre as tarefas candidatas, aquela que melhor se encaixa nas janelas deixadas pela programação das tarefas que já fazem parte do sequenciamento e com menor somatória de tempo de processamento. A partir daí, a extensão natural do Fits que explora várias operações por iteração utilizando as mesmas regras deu origem ao método BS deste trabalho. A avaliação do desempenho dos métodos propostos foi feita utilizando um conjunto de 1200 instâncias e 2 diferentes datas de entrega propostos em Chandra et al. (2009). Inicialmente, os resultados obtidos pelo LS foram comparados com dois outros métodos da literatura da mesma categoria. Os resultados mostraram que o LS obteve resultados muito melhores em tempo computacional razoavelmente equivalente. 


\begin{tabular}{|c|c|c|c|c|c|c|c|c|}
\hline \multicolumn{9}{|c|}{$d^{\prime \prime}$} \\
\hline \multicolumn{2}{|c|}{ Instância } & \multicolumn{3}{|c|}{$\overline{\mathrm{LS}}$} & \multicolumn{3}{|c|}{$\overline{B S}$} & \multirow{2}{*}{ \# Empates } \\
\hline$n$ & $m$ & $\overline{g a p}$ & $W$ & \# Vitórias & $\overline{g a p}$ & $W$ & \# Vitórias & \\
\hline$\overline{5}$ & $\overline{5}$ & 30.93 & 0.00 & $\overline{4}$ & -0.69 & 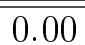 & $\overline{50}$ & $\overline{4}$ \\
\hline 5 & 10 & 34.83 & 0.00 & 2 & -1.59 & 0.00 & 50 & 1 \\
\hline 5 & 15 & 40.32 & 0.00 & 2 & -1.14 & 0.00 & 50 & 2 \\
\hline 5 & 20 & 55.26 & 0.00 & 1 & -0.74 & 0.00 & 50 & 1 \\
\hline 10 & 5 & 27.66 & 0.00 & 0 & -0.24 & 0.00 & 40 & 0 \\
\hline 10 & 10 & 35.35 & 0.00 & 0 & -1.12 & 0.00 & 33 & 0 \\
\hline 10 & 15 & 38.74 & 0.00 & 0 & -0.14 & 0.00 & 37 & 0 \\
\hline 10 & 20 & 44.04 & 0.00 & 0 & 0.18 & 0.00 & 27 & 0 \\
\hline 20 & 5 & 25.14 & 0.00 & 0 & -0.68 & 0.00 & 29 & 0 \\
\hline 20 & 10 & 30.84 & 0.00 & 1 & -0.45 & 0.00 & 29 & 0 \\
\hline 20 & 15 & 32.42 & 0.00 & 0 & -0.53 & 0.00 & 31 & 0 \\
\hline 20 & 20 & 34.93 & 0.00 & 0 & -1.01 & 0.00 & 32 & 0 \\
\hline 50 & 5 & 22.39 & 0.00 & 0 & -0.96 & 0.00 & 35 & 0 \\
\hline 50 & 10 & 23.32 & 0.00 & 0 & -2.86 & 0.00 & 40 & 0 \\
\hline 50 & 15 & 25.24 & 0.00 & 0 & -3.79 & 0.00 & 41 & 0 \\
\hline 50 & 20 & 26.11 & 0.00 & 0 & -3.51 & 0.00 & 42 & 0 \\
\hline 80 & 5 & 20.93 & 0.00 & 0 & -1.42 & 0.00 & 36 & 0 \\
\hline 80 & 10 & 21.41 & 0.00 & 0 & -4.02 & 0.00 & 42 & 0 \\
\hline 80 & 15 & 22.36 & 0.00 & 0 & -3.88 & 0.00 & 42 & 0 \\
\hline 80 & 20 & 21.99 & 0.00 & 0 & -4.51 & 0.00 & 43 & 0 \\
\hline 100 & 5 & 19.62 & 0.00 & 1 & -1.40 & 0.00 & 34 & 0 \\
\hline 100 & 10 & 20.30 & 0.00 & 0 & -3.42 & 0.00 & 41 & 0 \\
\hline 100 & 15 & 19.47 & 0.00 & 0 & -4.64 & 0.00 & 43 & 0 \\
\hline 100 & 20 & 20.80 & 0.00 & 0 & -4.84 & 0.00 & 44 & 0 \\
\hline & & 28.93 & 0.00 & 0.46 & -1.97 & 0.00 & 39.21 & - \\
\hline
\end{tabular}

Tabela 3.11: Resultados computacionais dos métodos LS e BS propostos neste capítulo considerando as 1200 instâncias e a data de entrega $d^{\prime \prime}$. Na tabela, cada entrada de $\overline{g a p}$ é a média dos gap relativos da soma dos adiantamentos e dos atrasos encontrados pelos métodos em relação aos resultados reportados por Chandra et al. (2009) em 50 instâncias com n tarefas e m máquinas. \# Empates é a quantidade de vezes que os dois métodos encontraram o mesmo valor de gap. W é o IDR da soma dos tempos de espera nesses casos. \# Vitórias é a quantidade de vezes em que o método conseguiu atingir ou superar os resultados reportados por Chandra et al. (2009).

Posteriormente, um subconjunto das instâncias foi utilizado para analisar e calibrar os parâmetros do método BS. A partir da definição dos parâmetros, os resultados computacionais para todo o conjunto foi comparado com os resultados reportados para a busca tabu de Chandra et al. (2009). O BS conseguiu atingir ou superar os resultados reportados em 2065 dos 2400 casos analisados, melhorando em média $-3.73 \%$ e $-1.97 \%$ os resultados de Chandra et al. (2009) para as duas diferentes datas de entrega consideradas, respectivamente.

Por fim, um solver exato foi aplicado sobre o modelo matemático proposto na Seção $3.1 \mathrm{em} \mathrm{um}$ subconjunto de instâncias de pequeno porte contendo 500 das 2400 instâncias do conjunto total. Em linhas gerais, enquanto o LS conseguiu encontrar valores ótimos para soma dos adiantamentos e atrasos em somente 16 dos 500 casos, o BS conseguiu encontrar em 455 dos 500 casos. Além disso, a média geral do gap em relação ao solver ficou em torno de $40 \%$ para o LS e em torno de $0.12 \%$ para o BS. 


\begin{tabular}{|c|c|c|c|c|c|c|c|c|}
\hline \multicolumn{9}{|c|}{$d^{\prime}$} \\
\hline \multicolumn{2}{|c|}{ Instância } & \multicolumn{3}{|c|}{$\mathrm{LS}$} & \multicolumn{3}{|c|}{$\overline{\mathrm{BS}}$} & \multirow{2}{*}{ \# Empates } \\
\hline$n$ & $m$ & $\overline{g a p}$ & $W$ & \# Vitórias & $\overline{g a p}$ & $W$ & \# Vitórias & \\
\hline 5 & 5 & 28.81 & 0.33 & 3 & 0.00 & 0.33 & 50 & 3 \\
\hline 5 & 10 & 42.11 & 0.50 & 2 & 0.00 & 0.50 & 50 & 2 \\
\hline 5 & 15 & 39.65 & 0.00 & 3 & 0.00 & 0.00 & 50 & 3 \\
\hline 5 & 20 & 59.79 & - & 0 & 0.00 & - & 50 & 0 \\
\hline 10 & 5 & 28.77 & - & 0 & 0.58 & - & 32 & 0 \\
\hline \multicolumn{2}{|c|}{ Média } & ב39.83 & 0.28 & 1.60 & "0.12 & 0.28 & 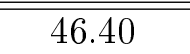 & \\
\hline
\end{tabular}

Tabela 3.12: Resultados computacionais dos métodos LS e BS propostos neste capítulo comparados com um solver ótimo executado sobre (3.1)-(3.10) considerando as instâncias de pequeno porte e a data de entrega $d^{\prime}$. Na tabela, cada entrada de $\overline{g a p}$ é a média dos gap relativos da soma dos adiantamentos e dos atrasos encontrados pelos métodos em relação aos resultados encontrados pelo solver ótimo em 50 instâncias com $n$ tarefas e $m$ máquinas. \# Empates é a quantidade de vezes que os dois métodos encontraram o mesmo valor de gap. W é o IDR da soma dos tempos de espera nesses casos. \# Vitórias é a quantidade de vezes em que o método conseguiu gap igual a zero.

\begin{tabular}{|c|c|c|c|c|c|c|c|c|}
\hline \multicolumn{9}{|c|}{$d^{\prime \prime}$} \\
\hline \multicolumn{2}{|c|}{ Instância } & \multicolumn{3}{|c|}{$\mathrm{LS}$} & \multicolumn{3}{|c|}{$\mathrm{BS}$} & \multirow{2}{*}{ \# Empates } \\
\hline$n$ & $m$ & $\overline{g a p}$ & $W$ & \# Vitórias & $\overline{g a p}$ & $W$ & \# Vitórias & \\
\hline 5 & 5 & 31.87 & 0.25 & 4 & 0.00 & 0.25 & 50 & 4 \\
\hline 5 & 10 & 37.56 & 0.00 & 1 & 0.00 & 0.00 & 50 & 1 \\
\hline 5 & 15 & 42.39 & 0.50 & 2 & 0.00 & 0.50 & 50 & 2 \\
\hline 5 & 20 & 56.75 & 1.00 & 1 & 0.00 & 1.00 & 50 & 1 \\
\hline 10 & 5 & 28.74 & - & 0 & 0.59 & - & 23 & 0 \\
\hline \multicolumn{2}{|c|}{ Média } & 39.46 & 0.44 & 1.60 & 0.12 & 0.44 & 44.60 & \\
\hline
\end{tabular}

Tabela 3.13: Resultados computacionais dos métodos LS e BS propostos neste capítulo comparados com um solver ótimo executado sobre (3.1)-(3.10) considerando as instâncias de pequeno porte e a data de entrega $d^{\prime \prime}$. Na tabela, cada entrada de $\overline{g a p}$ é a média dos gap relativos da soma dos adiantamentos e dos atrasos encontrados pelos métodos em relação aos resultados encontrados pelo solver ótimo em 50 instâncias com $n$ tarefas e $m$ máquinas. \# Empates é a quantidade de vezes que os dois métodos encontraram o mesmo valor de gap. W é o IDR da soma dos tempos de espera nesses casos. \# Vitórias é a quantidade de vezes em que o método conseguiu gap igual a zero. 


\section{Capítulo 4}

\section{Conclusões}

Esta tese aborda dois problemas de programação da produção, o Flexible Job Shop Scheduling Problem com flexibilidade de sequenciamento (FJSP-SF) e o Flowshop Scheduling Problem com tempos de espera (FSP-WT).

Em ambos os problemas, a metodologia utilizada foi a de inicialmente desenvolver um algoritmo construtivo do tipo list scheduling (LS) bem definido que explora características do problema em questão e então estender este algoritmo para um método Beam Search (BS). O BS proposto utiliza o LS de diferentes formas em todos os seus principais elementos: (1) expansão dos níveis da árvore de busca, (2) avaliação local dos candidatos e (3) avaliação global dos candidatos.

Os métodos propostos para o FJSP-SF foram comparados utilizando resultados de um solver exato executado sobre um modelo matemático da literatura para o problema. Além disso, o BS também foi comparado com instâncias do FJSP clássico contra algoritmos genéticos considerados estado da arte da literatura. Os resultados computacionais são encorajadores e mostram a eficiência dos métodos propostos para o problema. Mesmo lidando com instâncias que não são o foco do método, o BS conseguiu superar nas instâncias mais complexas os algoritmos genéticos para o FJSP da literatura.

Os métodos propostos para o FSP-WT também foram comparados contra outras heurísticas da literatura. O LS proposto conseguiu superar outros dois métodos da mesma categoria encontrados na literatura enquanto o BS conseguiu atingir ou superar os resultados reportados por Chandra et al. (2009) em 2065 dos 2400 casos analisados. Além disso, quando comparados com um solver exato aplicado ao modelo matemático para o problema, também proposto neste trabalho, os métodos se mostraram promissores e em particular, o método BS conseguiu atingir valores ótimos em 455 dos 500 casos avaliados.

Em linhas gerais, devido aos resultados computacionais encorajadores, a metodologia para o desenvolvimento dos métodos se mostrou robusta visto que foi muito bem sucedida em dois diferentes problemas de programação da produção, utilizando pouco tempo computacional e superando os métodos da literatura.

\subsection{Trabalhos Futuros}

Para o FSP-WT, a análise dos métodos propostos em cenários com diferentes objetivos de minimização, como o atraso total ou o makespan, a comparação dos métodos propostos contra ótimos para instâncias pequenas e a extensão dos métodos para o problema que possui datas de liberação são opções de trabalhos futuros.

Para o FJSP-SF, a adaptação dos métodos propostos em diferentes cenários do FJSP clássico são tópicos para futuras pesquisas.

No geral, utilizar a metodologia abordada no desenvolvimento dos métodos desta tese em problemas diferentes dos de programação da produção é uma proposta intrigante para pesquisas futuras. 


\section{Referências Bibliográficas}

Agnetis et al.(2014) Alessandro Agnetis, Paolo Detti e Marco Pranzo. The list scheduling algorithm for scheduling unreliable jobs on two parallel machines. Discrete Applied Mathematics, 165:2-11. Citado na pág. 2, 3

Akeb et al.(2009) Hakim Akeb, Mhand Hifi e Rym M'Hallah. A beam search algorithm for the circular packing problem. Comput. Oper. Res., 36:1513-1528. ISSN 0305-0548. Citado na pág. 2

Akeb et al.(2011) Hakim Akeb, Mhand Hifi e Stéphane Negre. An augmented beam searchbased algorithm for the circular open dimension problem. Comput. Ind. Eng., 61:373-381. ISSN 0360-8352. Citado na pág. 2

Antunes Júnior et al.(1989) J. A. V. Antunes Júnior, F. J. Kliemann Neto e J. Fensterseifer. Considerações críticas sobre a evolução das filosofias de administração da produção: Do just-incase ao just-in-time. Revista de Administração de Empresas, 29:49-64. Citado na pág. 1

Barnes e Chambers(1996) J. W. Barnes e J. B. Chambers. Flexible job shop scheduling with tabu search. Graduate program in operations research and industrial engineering, University of texas. Citado na pág. 27

Birgin et al.(2014) E. G. Birgin, P. Feofiloff, C. G. Fernandes, E. L. de Melo, M. T. I. Oshiro e D. P. Ronconi. A milp model for an extended version of the flexible job shop problem. Optimization Letters, 8(4):1417-1431. Citado na pág. xi, 7, 9, 23, 24, 27, 28, 29

Birgin et al.(2015) E.G. Birgin, J.E. Ferreira e D.P. Ronconi. List scheduling and beam search methods for the flexible job shop scheduling problem with sequencing flexibility. European Journal of Operational Research, 247(2):421-440. Citado na pág. ix, 2, 6, 8

Blum et al.(2009) Christian Blum, Maria J. Blesa e Manuel López-Ibáñez. Beam search for the longest common subsequence problem. Comput. Oper. Res., 36:3178-3186. Citado na pág. 2

Bouazza et al.(2017) W. Bouazza, Y. Sallez e B. Beldjilali. A distributed approach solving partially flexible job-shop scheduling problem with a q-learning effect. IFAC-PapersOnLine, 50 (1):15890 - 15895. 20th IFAC World Congress. Citado na pág. 8

Bozejko et al.(2012) Wojciech Bozejko, Zdzilaw Hejducki, Mariusz Uchronski e Mieczyslaw Wodecki. Solving the flexible job shop problem on multi-gpu. Procedia Computer Science, 9:2020 2023. Proceedings of the International Conference on Computational Science, ICCS 2012. Citado na pág. 8

Brandimarte(1993) P. Brandimarte. Routing and scheduling in a flexible job shop by tabu search. Annals of Operations Research, 41:157-183. Citado na pág. 7, 27

Chandra et al.(2009) P. Chandra, P. Mehta e D. Tirupati. Permutation flow shop scheduling with earliness and tardiness penalties. International Journal of Production Research, 47(20): 5591-5610. Citado na pág. xii, $36,53,54,55,57,58,59,61$ 
Chen et al.(1999) H. Chen, J. Ihlow e C. Lehmann. A genetic algorithm for flexible job-shop scheduling. Proceedings of the IEEE International Conference on Robotics and Automation, 2: 1120-1125. Citado na pág. 32

Chiavenato(2004) I. Chiavenato. Introdução à Teoria Geral da Administração: Edição Compacta. CAMPUS. Citado na pág. 1

Cormen et al.(2009) T. H. Cormen, C. E. Leiserson, R. L. Rivest e C. Stein. Introduction to Algorithms. The MIT Press, 3rd edição. Citado na pág. 13

Dauzère-Pérès e Paulli(1997) S. Dauzère-Pérès e J. Paulli. An integrated approach for modeling and solving the general multiprocessor job-shop scheduling problem using tabu search. Annals of Operations Research, 70:281-306. Citado na pág. 7, 27

Fard et al.(2014) Hamid Mohammadi Fard, Radu Prodan e Thomas Fahringer. Multi-objective list scheduling of workflow applications in distributed computing infrastructures. Journal of Parallel and Distributed Computing, 74(3):2152-2165. Citado na pág. 2, 3

Fattahi et al.(2007) P. Fattahi, M. Mehrabad e F. Jolai. Mathematical modeling and heuristic approaches to flexible job shop scheduling problems. Journal of Intelligent Manufacturing, 18: 331-342. Citado na pág. 7

Fernandez-Viagas e Framinan(2017) Victor Fernandez-Viagas e Jose M. Framinan. A beamsearch-based constructive heuristic for the pfsp to minimise total flowtime. Computers $\& 3$ Operations Research, 81:167 - 177. Citado na pág. 2

Fernandez-Viagas et al.(2016) Victor Fernandez-Viagas, Manuel Dios e Jose M. Framinan. Efficient constructive and composite heuristics for the permutation flowshop to minimise total earliness and tardiness. Computers $\mathscr{E} 3$ Operations Research, 75:38 - 48. Citado na pág. 36

Fisher e Thompson(1963) H. Fisher e G. L. Thompson. Probabilistic learning combinations of local job-shop scheduling rules. Em G.L. Thompson J.F. Muth, editor, Industrial Scheduling, páginas 225-251. Prentice-Hall, Englewood Cliffs, NJ. Citado na pág. 7

Gan e Lee(2002) P. Y. Gan e K. S. Lee. Scheduling of flexible-sequenced process plans in a mould manufacturing shop. International Journal of Advanced Manufacturing Technology, 20:214-222. Citado na pág. 6

Garey et al.(1976) M. Garey, D. Johnson e R. Sethi. The complexity of flowshop and jobshop scheduling. Mathematics of Operations Research, 1(2):117-129. Citado na pág. 2, 7

Gendreau(2003) M. Gendreau. An Introduction to Tabu Search, volume 57. Springer. Citado na pág. 2

Gutiérrez e García-Magariño(2011) C. Gutiérrez e I. García-Magariño. Modular design of a hybrid genetic algorithm for a flexible job-shop scheduling problem. Knowledge-Based Systems, 24(1):102-112. Citado na pág. 7

Hall e Posner(1991) Nicholas G. Hall e Marc E. Posner. Earliness-tardiness scheduling problems, i: Weighted deviation of completion times about a common due date. Operations Research, 39 (5):836-846. Citado na pág. 2, 35

Hendel e Sourd(2007) Yann Hendel e Francis Sourd. An improved earlinesstardiness timing algorithm. Computers \& Operations Research, 34(10):2931 - 2938. Citado na pág. 36, 41, 42

Ho et al.(2007) N. B. Ho, J. C. Tay e E. M.-K. Lai. An effective architecture for learning and evolving flexible job-shop schedules. European Journal of Operational Research, 179(2):316-333. Citado na pág. 7 
Hurink et al.(1994) J. Hurink, B. Jurish e M. Thole. Tabu search for the job-shop scheduling problem with multi-purpose machines. OR-Spektrum, 15(4):205-215. Citado na pág. 27

Jia et al.(2003) H. Z. Jia, A. Y. C. Nee, J. Y. H. Fuh e Y. F. Zhang. A modified genetic algorithm for distributed scheduling problems. International Journal of Intelligent Manufacturing, 14(3): 351-362. Citado na pág. 32

Kanet(1981) John J. Kanet. Minimizing the average deviation of job completion times about a common due date. Naval Research Logistics Quarterly, 28(4):643-651. Citado na pág. 36

Kim et al.(2003) Y. K. Kim, K. Park e J. Ko. A symbiotic evolutionary algorithm for the integration of process planning and job shop scheduling. Computers $\&$ Operations Research, 30: 1151-1171. Citado na pág. 6

Lee et al.(2012) S. Lee, I. Moon, H. Bae e J. Kim. Flexible job-shop scheduling problems with and/or precedence constraints. International Journal of Production Research, 50:1979-2001. Citado na pág. 6

Lenstra e Kan(1979) J. K Lenstra e A. H. G. Rinnoy Kan. Computational complexity of discrete optimisation problems. Annals of Discrete Mathematics, 4:121-140. Citado na pág. 2, 7

Lowerre(1976) B. T. Lowerre. The harpy speech recognition system. Tese de Doutorado, Carnegie Mellon University, Pittsburgh, PA, USA. Citado na pág. 2, 8

Mastrolilli e Gambardella(2000) M. Mastrolilli e L. M. Gambardella. Effective neighbourhood functions for the flexible job shop problem. Journal of Scheduling, 3:3-20. Citado na pág. 7

Mejía e Niño(2017) Gonzalo Mejía e Karen Niño. A new hybrid filtered beam search algorithm for deadlock-free scheduling of flexible manufacturing systems using petri nets. Computers 83 Industrial Engineering, 108:165 - 176. Citado na pág. 2

Moslehi et al.(2009) G. Moslehi, M. Mirzaee, M. Vasei, M. Modarres e A. Azaron. Two-machine flow shop scheduling to minimize the sum of maximum earliness and tardiness. International Journal of Production Economics, 122(2):763 - 773. Citado na pág. 36

Nawaz et al.(1983) Muhammad Nawaz, E Emory Enscore e Inyong Ham. A heuristic algorithm for the m-machine, n-job flow-shop sequencing problem. Omega, 11(1):91 - 95. Citado na pág. 36

Nuijten e Aarts(1996) W.P.M. Nuijten e E.H.L. Aarts. A computational study of constraint satisfaction for multiple capacitated job shop scheduling. European Journal of Operational Research, 90(2):269 - 284. Citado na pág. 7

Pezzella et al.(2008) F. Pezzella, G. Morganti e G. Ciaschetti. A genetic algorithm for the flexible job-shop scheduling problem. Computers \& Operations Research, 35:3202-3212. Citado na pág. 7, $27,28,29,32$

Pinedo(2008) M. Pinedo. Scheduling: theory, algorithms, and systems. Springer. Citado na pág. 2, 35

PNEAC(2015) PNEAC. Printers national environmental assistance center (pneac), acessado em 9 de fevereiro de 2015, 2015. URL http://www.pneac.org. Citado na pág. 5

Rakrouki et al.(2012) Mohamed Ali Rakrouki, Talel Ladhari e Vincent T'kindt. Coupling genetic local search and recovering beam search algorithms for minimizing the total completion time in the single machine scheduling problem subject to release dates. Computers 83 Operations Research, 39:1257-1264. ISSN 0305-0548. Citado na pág. 2, 8

Rubins(1978) S. Rubins. The ARGOS Image Understanding System. Tese de Doutorado, Pittsburgh, PA, USA. Citado na pág. 8 
Sabuncuoglu e Bayiz(1999) I. Sabuncuoglu e M. Bayiz. Job shop scheduling with beam search. European Journal of Operational Research, 118(2):390-412. Citado na pág. 3, 8, 17

Sakuraba et al.(2009) C. S. Sakuraba, D. P. Ronconi e F. Sourd. Scheduling in a two-machine flowshop for the minimization of the mean absolute deviation from a common due date. Computers E3 Operations Research, 36:60-72. Citado na pág. 35, 36, 41, 42, 48, 54

Sarper(1995) Hüseyin Sarper. Minimizing the sum of absolute deviations about a common due date for the two-machine flow shop problem. Applied Mathematical Modelling, 19(3):153 - 161. Citado na pág. 36

Tang et al.(2010) Xiaoyong Tang, Kenli Li, Guiping Liao e Renfa Li. List scheduling with duplication for heterogeneous computing systems. Journal of Parallel and Distributed Computing, 70 (4):323-329. Citado na pág. 2, 3

Valente e Alves(2004) J. M. S. Valente e R. A. F. S. Alves. Beam search algorithms for the early/tardy scheduling problem with release dates. FEP Working Papers 143, Universidade do Porto, Faculdade de Economia do Porto. Citado na pág. 2

Vélez-Gallego et al.(2016) Mario C. Vélez-Gallego, Jairo Maya e Jairo R. Montoya-Torres. A beam search heuristic for scheduling a single machine with release dates and sequence dependent setup times to minimize the makespan. Computers $\&$ Operations Research, 73:132 - 140. Citado na pág. 2

Wan e Yuan(2013) Long Wan e Jinjiang Yuan. Single-machine scheduling to minimize the total earliness and tardiness is strongly np-hard. Operations Research Letters, 41(4):363 - 365. Citado na pág. 35

Wang e $\operatorname{Lim}(2007)$ F. Wang e A. Lim. A stochastic beam search for the berth allocation problem. Decis. Support Syst., 42:2186-2196. ISSN 0167-9236. Citado na pág. 2

Wang et al.(2012) L. Wang, G. Zhou, Y. Xu, S. Wang e M. Liu. An effective artificial bee colony algorithm for the flexible job-shop scheduling problem. The International Journal of Advanced Manufacturing Technology, 60(1):303-315. Citado na pág. 7

Yuan e Xu(2012) Y. Yuan e H. Xu. Hhs/lns: An integrated search method for flexible job shop scheduling. Em Evolutionary Computation (CEC), 2012 IEEE Congress on, páginas 1-8. Citado na pág. 7

Zeng et al.(2010) J. Zeng, S. Jackson, I.-J. Lin, M. Gustafson, E. Hoarau e R. Mitchell. On-demand digital print operations: A simulation based case study. Technical report, Hewlett-Packard. Citado na pág. 5

Özgüven et al.(2010) C. Özgüven, L. Özbakir e Y. Yavuz. Mathematical models for job-shop scheduling problems with routing and process plan flexibility. Applied Mathematical Modelling, 34(6):1539-1548. Citado na pág. 7 